\title{
Preliminary Analysis of the Gradient Field Imploding Liner Fusion Propulsion Concept
}

\author{
M. LaPointe ${ }^{1}$ and R. Adams ${ }^{2}$ \\ NASA Marshall Space Flight Center, Huntsville, AL 35812 \\ J. Cassibry ${ }^{3}$ and M. Zweiner ${ }^{4}$ \\ University of Alabama, Huntsville \\ J. Gilland, ${ }^{5}$ \\ Ohio Aerospace Institute, Cleveland $\mathrm{OH}$
}

\begin{abstract}
The advancement of human deep space exploration requires the continued development of energetic inspace propulsion systems, advancing from current chemical engines to nuclear thermal rockets to future high energy concepts such as nuclear fusion. This paper presents the initial results of a NASA Innovative Advanced Concepts (NIAC) Phase I study funded to investigate the feasibility of a new pulsed fusion propulsion concept based on the rapid implosion of a fuel target injected at high velocity into a strong stationary magnetic field. The proposed concept takes advantage of the significant advances in terrestrial magneto-inertial fusion designs while attempting to mitigate the most common engineering impediments to in-space propulsion applications. A semi-analytic numerical model used to estimate target compression physics and energy release is presented, leading to estimates for engine performance. A preliminary vehicle design concept is outlined, and representative trajectory analyses for rapid Mars and Saturn missions are provided. The paper concludes with an overview of proposed next steps for theoretical and experimental validation of the concept.
\end{abstract}

\section{Background}

The advancement of human deep space exploration requires the continued development of energetic in-space propulsion systems, from current chemical engines to nuclear thermal rockets to future high energy concepts such as nuclear fusion. This NASA Innovative Advanced Concepts (NIAC) Phase I study was funded to investigate the feasibility of an innovative approach toward highly energetic pulsed fusion propulsion. Several prior concept studies have proposed the conversion of fusion energy for in-space propulsion, ranging from laser ignited fusion systems such as Gevaltig [1] and VISTA [2], to the British Interplanetary Society’s Daedalus concept [3] and its more recent incarnation under Project Icarus [4], to steady-state spherical torus fusion systems [5]. Other NIAC studies have also evaluated several innovative fusion concepts, including the acceleration and compression of FRC plasmas in time changing magnetic fields [6], magnetically driven liners imploding onto plasma targets [7], and high current Z-pinch compression of material liners onto fission-fusion fuel targets [8]. While each of these studies firmly established the potential benefits of fusion systems for interplanetary travel, they also identified significant challenges in successfully engineering such systems for spacecraft propulsion. The concept outlined in this report builds on the lessons learned from these prior activities, approaching the quest for fusion powered propulsion through an innovative variation of magneto-inertial fusion concepts developed for terrestrial power applications.

\section{A. Concept Description}

The rapid magnetic compression of fusion fuel targets remains an area of active development [9-11]. To successfully implode the target and trigger fusion reactions, a pulsed high current discharge in a surrounding magnetic field coil generates a rapidly changing axial magnetic field, $\mathrm{dB}_{\mathrm{z}} / \mathrm{dt}$, which induces a counter-propagating

\footnotetext{
${ }^{1}$ Manager, Space Technology Development Branch; Associate Fellow

${ }^{2}$ Senior Research Engineer, Propulsion Research Branch; Associate Fellow

${ }^{3}$ Associate Professor, Department of Mechanical and Aerospace Engineering; Lifetime Associate Member

${ }^{4}$ Research Scientist, Department of Mechanical and Aerospace Engineering

${ }^{5}$ Senior Scientist \& Team Lead, Space Science \& Exploration Technology; Associate Fellow
} 
current in the conducting outer liner of a centrally aligned cylindrical target. The inductively driven liner current rapidly implodes the liner radially inward, compressing the fuel to the densities and temperatures required for fusion. A significant energy loss mechanism observed during liner compression and ignition of the resulting plasma is electron thermal conduction, which is mitigated by the use of strong magnetic fields within the target to suppress cross field thermal conduction losses, generating higher plasma temperatures at lower implosion velocities [12].

While promising, there are several challenges associated with imploding liner fusion concepts that must be addressed in the context of a propulsion system. The requirement of short duration, high current pulses to produce a rapidly changing magnetic field for target compression precludes the use of superconducting magnets, resulting in significant resistive losses and coil heating. Related issues include the need for capacitive or inductive power supplies, pulse forming networks, and robust, long life switches capable of repetitively channeling several MA of pulsed current into the magnetic field coil. Repetitive and accurate target replacement within the coil after each pulse, together with mitigating liner or other target material debris impacts to the chamber walls, presents additional engineering challenges. Targets must be designed to suppress potential instabilities which may occur at the liner-fuel interface during compression, either by judicious choice of liner materials and thickness in solid target liners or the rotation of liquid liners prior to compression. Preheating the target fuel has been shown to improve ignition, and a method to efficiently deliver an energy pulse to the target prior to main compression must be incorporated. Finally, coupling the energy released in a successful target fusion event to the spacecraft without incurring structural or material damage requires directing the rapidly expanding plasma out of the chamber, using either a magnetic nozzle or causing the event to occur downstream where it can impact against a pusher plate to provide thrust.

The proposed concept takes advantage of the significant advances in terrestrial magneto-inertial fusion (MIF) designs while attempting to mitigate the potential engineering impediments to in-space propulsion applications. Methods to induce a rapid radial compression in a stationary central target typically focus on z-pinch or $\theta$-pinch geometries. In the z-pinch, a very high current pulse is sent through a conducting liner coating a cylindrical fusion target; the axial current induces an azimuthal magnetic field, and the combination creates a radial Lorentz force that rapidly compresses the target to high density and temperature. In a $\theta$-pinch, current is pulsed through a drive coil surrounding the central target; the pulsed current creates a time changing axial magnetic field, which in turn induces an azimuthal current in the conducting target liner. The combination of strong axial magnetic field and induced azimuthal current combine to again provide a rapid radial Lorentz force compression of the target. Equivalently, the compression mechanism in each case can be considered the result of a rapid buildup of external magnetic field pressure external to the conducting liner; which inhibits field penetration into the target. The external pressure significantly exceeds the target fuel internal pressure, forcing a rapid radial compression until the pressures equilibrate at high internal densities and temperatures.

Prior studies have evaluated the application of z-pinch geometries for in-space propulsion [8], and several ground-based fusion experiments are based on the z-pinch approach [13.14]. Although options to incorporate zpinch physics into an in-space propulsion system continue to be explored, the current concept instead utilizes a geometry more closely aligned with the $\theta$-pinch. An overview of $\theta$-pinch operation is provided in Fig. 1 below [15]; as noted, current sent through the drive coil induces an opposing azimuthal current in the stationary central target liner, and the combination of increasing axial magnetic field and induced azimuthal current provide rapid radial compression.

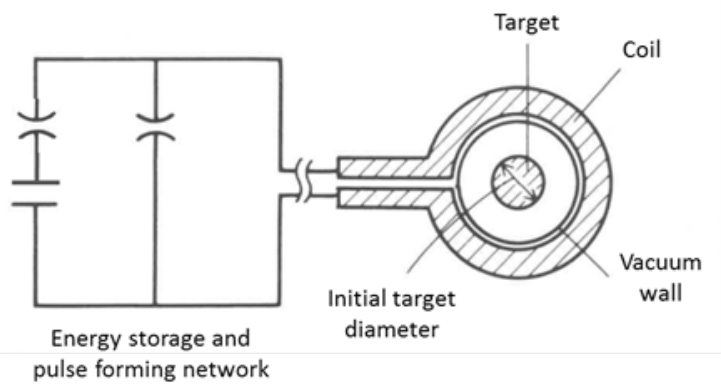

Fig. 1 Pulsed $\theta$-pinch operation. Adapted from [15]

The approach investigated in the current study replaces the pulsed, high current magnetic field coil and stationary central target with a fast moving target fired axially into a static, high gradient magnetic field. This essentially decomposes the time changing derivative of the axial magnetic field into partial derivatives associated with an axial magnetic field gradient and an axial velocity: 


$$
\frac{\mathrm{dB}_{\mathrm{z}}}{\mathrm{dt}}=\frac{\partial \mathrm{B}_{\mathrm{z}}}{\partial \mathrm{z}} \frac{\partial \mathrm{z}}{\partial \mathrm{t}}=\mathrm{v}_{\mathrm{z}} \frac{\partial \mathrm{B}_{\mathrm{z}}}{\partial \mathrm{z}}
$$

As such, a target fired at high axial velocity into a steep magnetic field gradient will effectively experience a rapidly changing axial magnetic field and undergo the same inductive compression as a stationary target at the center of a rapidly pulsed magnetic field. A conceptual overview of this gradient field imploding liner system is provided in Fig. 2 below, forming the basis for the study outlined in this paper.

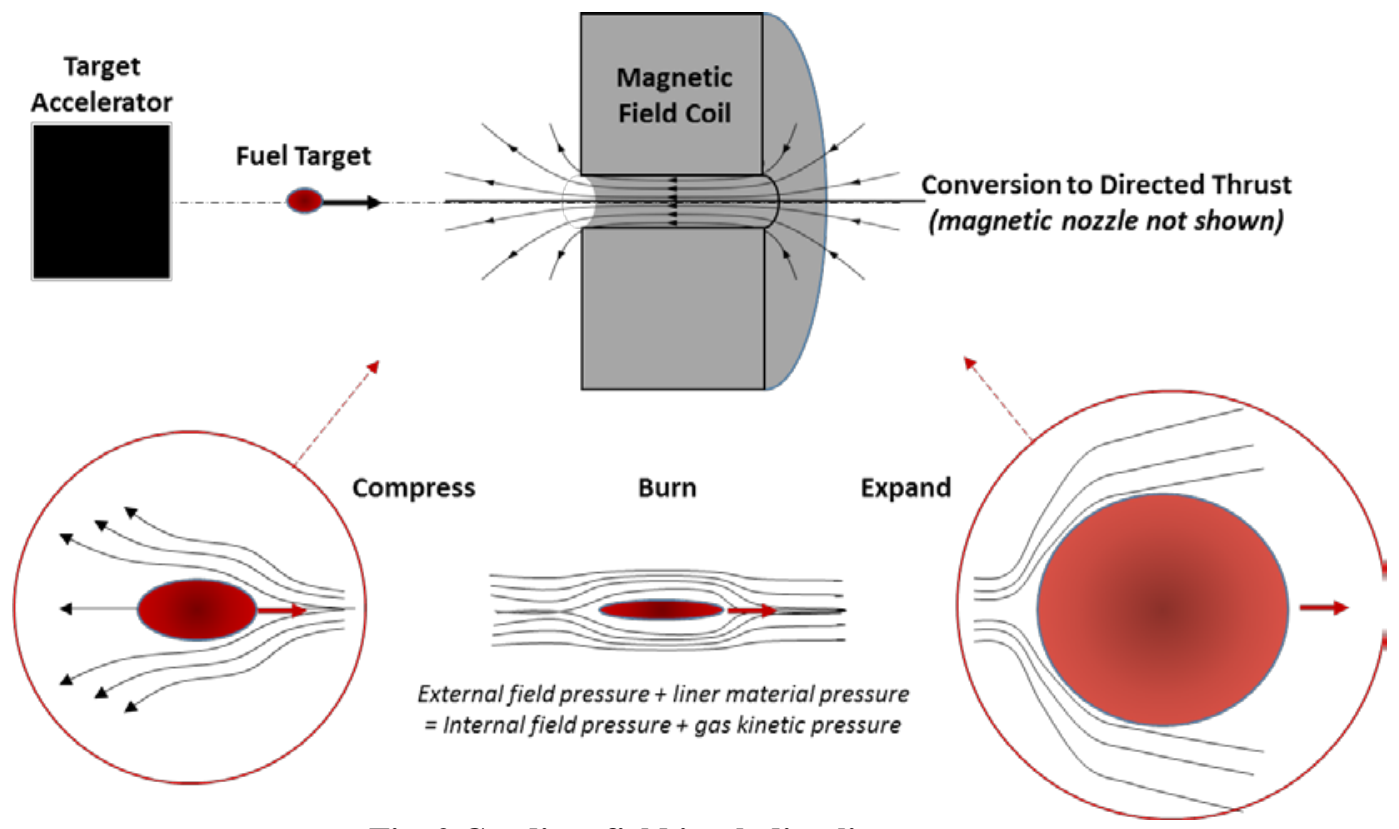

Fig. 2 Gradient field imploding liner concept

\section{B. Benefits and Challenges}

Shifting the onus of rapid target compression from a pulsed, high current coil to a target accelerator and a static high gradient magnetic field offers several potential advantages for in-space propulsion. Eliminating the need to rapidly pulse the magnetic field coil allows the use of energy efficient superconducting field coils, which in turn reduces energy storage requirements, coil thermal losses and associated radiator requirements. The field coils can be shaped to provide strong upstream field gradients, a high field mid-section to enhance target fuel burning, and a magnetic nozzle at the downstream exit plane to convert the rapidly expanding plasma into directed thrust. Target acceleration may be accomplished using one of several possible approaches, including inductive acceleration or laser ablation, the latter also offering a possible method for preheating the target fuel. Electron and ion radial thermal losses can be suppressed by strong internal magnetic fields trapped within the target during compression, reducing energy losses and improving target gain. The linear geometry of the system, together with the axial motion of the target as it enters, compresses, burns, and expands into the magnetic nozzle region, lends itself more naturally to repetitively pulsed in-space propulsion, easing design issues associated with target placement and energy transfer to the vehicle.

To realize these potential benefits, a number of significant challenges have been addressed during this study to determine the initial feasibility of the concept. These include modeling the magnetic field geometries and axial gradients required for fuel target compression, evaluating accelerator concepts to achieve high target velocities, evaluating initial target fuels and design options, and evaluating methods to convert the expanding high temperature plasma into directed thrust. Analytic and numerical models have been developed to simulate and understand target pellet compression and burn physics, which in turn feed back into target accelerator concepts, magnetic field designs, and mission performance parameters. Preliminary mission trajectory analysis and vehicle designs have been developed to guide system performance requirements and quantify potential benefits for crewed or robotic solar system and deep space exploration. Each of these areas are discussed in the following sections, which describes the methods, research status, and initial results that underpin the determination of concept viability. 


\section{Approach}

The purpose of this study is to investigate the dynamics and potential performance of a conceptual system that can rapidly inject, compress, and burn a fusion fuel target, and efficiently exhaust the resulting high temperature plasma, in a configuration suitable for in-space propulsion applications. Taking advantage of the experience gained by international MIF research programs, the concept seeks to replicate well known static target compression physics in a novel, dynamic system. As terrestrial systems advance toward breakeven, the target designs and field requirements used to reach these higher yields can be readily incorporated into this innovative in-space system design.

\section{A. Target Design}

For this initial evaluation, a cylindrical pellet with deuterium-tritium (D-T) fuel and a conductive liner has been chosen both for simplicity of modeling, and for consistency with current terrestrial MIF fusion experiments. D-T fuels have a higher fusion cross section at lower ignition temperatures, making them a standard fuel of choice for most terrestrial experiments. Figure 3 below lists several fusion reactions with their corresponding energy release, and a plot of reaction cross section vs. center of mass energy [16].

\begin{tabular}{|c|c|}
\hline Reaction & Energy Release \\
\hline$D+T \rightarrow \alpha+n$ & $17.59 \mathrm{MeV}$ \\
\hline$D+D \rightarrow T+p$ & $4.04 \mathrm{MeV}$ \\
\hline$D+D \rightarrow{ }^{3} \mathrm{He}+n$ & $3.27 \mathrm{MeV}$ \\
\hline$D+{ }^{3} \mathrm{He} \rightarrow \alpha+p$ & $18.35 \mathrm{MeV}$ \\
\hline$D+{ }^{6} \mathrm{Li} \rightarrow 2 \alpha$ & $22.374 \mathrm{MeV}$ \\
\hline$D+{ }^{6} \mathrm{Li} \rightarrow p+{ }^{7} \mathrm{Li}$ & $5.026 \mathrm{MeV}$ \\
\hline$D+{ }^{6} \mathrm{Li} \rightarrow n+{ }^{7} \mathrm{Be}$ & $3.38 \mathrm{MeV}$ \\
\hline$p+{ }^{11} B \rightarrow 3 \alpha$ & $8.68 \mathrm{MeV}$ \\
\hline$n+{ }^{6} \mathrm{Li} \rightarrow T+\alpha$ & $4.86 \mathrm{MeV}$ \\
\hline$n+{ }^{7} \mathrm{Li} \rightarrow T+\alpha+n$ & $-2.87 \mathrm{MeV}$ \\
\hline
\end{tabular}

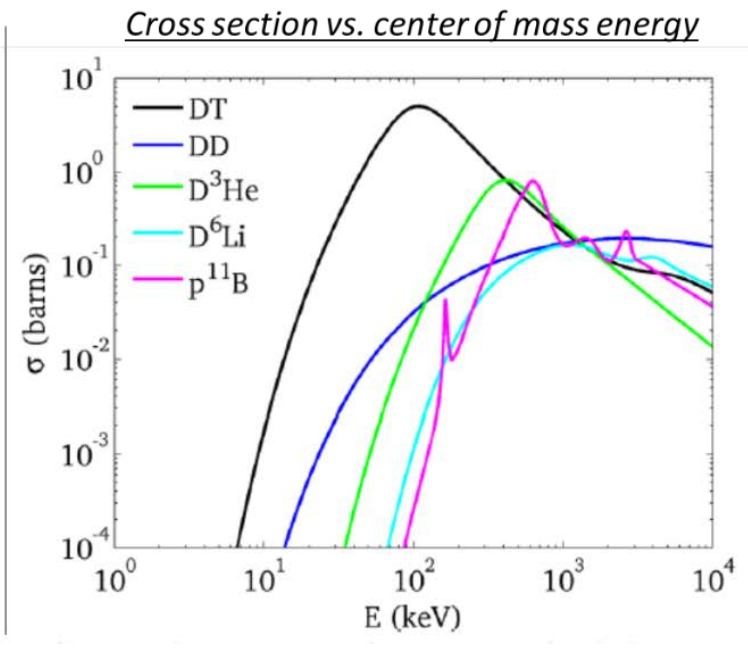

Fig. 3 Standard fusion reactions, energy release, and cross sections. Adapted from [16].

Several low atomic number coating materials have previously been investigated as conductive target liners, including beryllium, aluminum and lithium [17]. For the inductively driven target compression under consideration, the liner material serves to carry the induced azimuthal current, interacting with the applied axial magnetic field to generate a radial Lorentz force and rapidly compress the target. The heavier liner shell also provides momentum to the imploding target, stagnating on axis to provide longer confinement and burn times for more efficient fuel conversion.

Several ground based experiments and numerical studies have shown that the ratio of target radius to liner thickness (the aspect ratio, AR) plays a role in the evolution of disruptive Rayleigh-Taylor instabilities:

$$
\mathrm{AR}=\frac{\mathrm{R}}{\Delta \mathrm{R}}
$$

where $\mathrm{R}$ is the outer radius of the target (including liner), and $\Delta \mathrm{R}$ is the liner thickness. AR values $\leq 6$ have been shown to delay the onset of Rayleigh-Taylor instabilities [18.19], which if left unchecked will significantly limit target convergence and enhance material mixing between the liner and target layers. Enhanced mixing leads to a substantial reduction in fusion burnup due to high $\mathrm{Z}$ poisoning of the mixing layer and faster thermal conduction losses. As such, most targets employ liner coatings that satisfy this aspect ratio to improve target compression and heating. Additional methods to reduce the Rayleigh-Taylor instability have also been investigated, including the use of thin dielectric coatings over metallic liners [19], which appears to significantly improve converging target uniformity. While not evaluated in this study, dielectric coating of metallic liners can be incorporated into future, more detailed models and remains an area for later investigation. 
A key parameter for achieving fusion conditions is the areal density of the target, expressed as $\rho R$, where $\rho$ is the fuel density and $R$ is the radius at maximum compression. Values of $\rho R$ as a function of temperature required to achieve net energy gain are plotted in Lindl-Widner diagrams, an example of which is shown in Fig. 4 for cylindrical D-T fuel at stagnation [20].

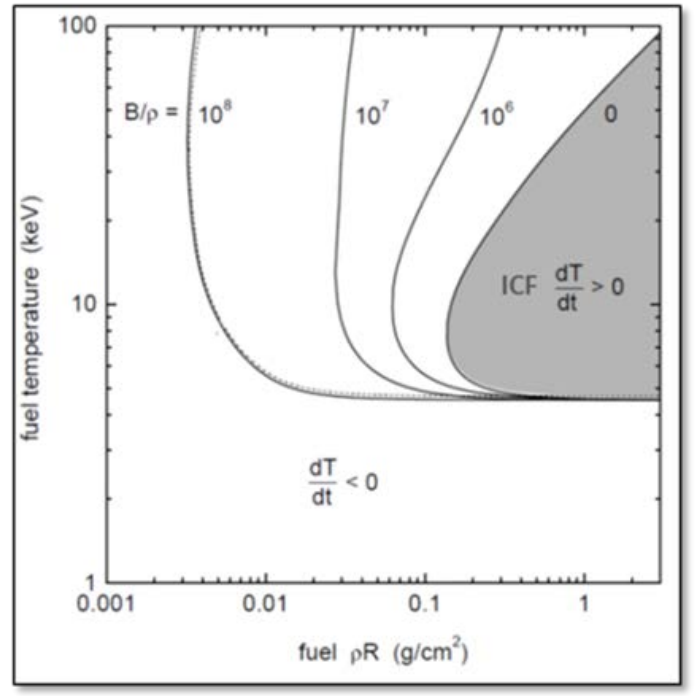

Fig. 4 Lindl-Widner diagram for D-T cylinder at stagnation. Adapted from [20].

The shaded region shows the area of net gain in pure inertial confinement fusion (ICF) without internal magnetic fields. The solid lines demarking areas to the left of this region show $\rho R$, $T$ parameters required for net energy gain in cases where the targets have internal magnetic fields (displayed in the figure as values of $B / \rho$ ). Such plots show that incorporating strong magnetic fields within the fuel can significantly reduce the value of $\rho \mathrm{R}$ required for net energy gain at a given temperature; this in turn implies a less stringent requirement on the final fuel density and radius at compression, reducing the energy required to compress the target. In current MIF experiments, applied magnetic fields of a few to several $\mathrm{T}$ are often used as the seed fields to produce compressed fields of $10^{2}-10^{3} \mathrm{~T}$ during the brief period of target implosion. Assuming an axial magnetic field is present and remains trapped within the cylindrical fuel target during compression, the field strength will be significantly increased during compression via the conservation of magnetic flux. These strong axial magnetic fields can trap charged $\alpha$ particles produced during the fusion process to enhance fuel heating, and in addition serve to reduce the loss of energy through radial electron and ion thermal conduction. This is the major advantage of MIF concepts over pure ICF, and why these systems are being actively investigated to provide terrestrial fusion power. The concept discussed in this paper likewise incorporates the use of seed magnetic fields to enhance the performance of the system.

In addition to pure fusion targets, prior studies have also investigated fission-fusion hybrid targets for magnetically imploding systems [21], including the recent NIAC effort to develop a pulsed fission-fusion z-pinch system [8]. In that concept, the target contained a central cylinder of deuterium-tritium (D-T) material, surrounded by a ${ }^{238} \mathrm{U}$ cylindrical sheath, which in turn is surrounded by a cylindrical lithium sheath. Upon implosion, the D-T mixture is compressed and a limited number of fusion reactions begin to take place; the resulting fast thermonuclear neutrons bombard the surrounding ${ }^{238} \mathrm{U}$ and induce fission, which in turn increases the fusion yield of the D-T core. Neutrons from both fission and fusion reactions are reflected and moderated by the surrounding lithium liner, reducing neutron escape and damage to surrounding structures. In this hybrid design, fusion neutrons result in a more complete burn of the fissile fuel, sustaining energy release. This sustained release extends the compression of the fusion reactants, yielding more fusion reactions, which in turn release more neutrons for more fissile material consumption. This synergy has been observed in the development of other fission-fusion devices, leading to more complete fuel burn up and allowing fusion ignition to be achieved with lower initial energy input than pure D-T targets. For the proposed concept, this would also translate into less severe requirements on the initial pellet velocities generated by the accelerator or on the strength of the gradient magnetic field. Pending a determination of the initial feasibility of the concept, a hybrid target remains a viable option to be investigated. 


\section{B. Target Accelerator Concepts}

Maintaining a static magnetic field with constant currents in superconducting coils simplifies the problem of repetitively pulsed high current coil discharges and associated coil heating, but introduces a new challenge in the design of the pellet accelerator. To induce rapid radial compression as the pellet enters the gradient magnetic field requires a high initial pellet velocity of several $\mathrm{km} / \mathrm{s}$. Several options were considered for the pellet accelerator, including gas guns, rail guns, electrothermal and electromagnetic accelerator concepts, and laser ablation acceleration. The dual requirements of efficient pellet acceleration to velocities of several $\mathrm{km} / \mathrm{s}$ and repetitive, long life operation in a vacuum environment reduced the initial set of options to the conceptual electromagnetic macron accelerator proposed by Kirtley [22], electrothermal accelerators proposed to accelerate fuel pellets for tokomak systems [23], and laser ablation concepts also proposed for use in refueling tokomak and other magnetic confinement fusion systems [24-26]. Of these, the macron system appears capable of accelerating gram-size pellets to the required velocities but has not yet been demonstrated; electrothermal accelerators working via ablative arcs have been demonstrated to achieve a few $\mathrm{km} / \mathrm{s}$ with gram size pellets, and remain a potential option. Laser ablation employs a high power laser pulse to ablate material at high velocity from one end of the pellet, causing the remaining mass to accelerate in the opposite direction. Models and initial experiments indicate accelerations of up to $200-\mathrm{km} / \mathrm{s}$ for mm-size DT pellets using modest laser intensities of $10^{14} \mathrm{~W} / \mathrm{cm}^{2}$, readily achieved by commercially available $\mathrm{CO}_{2}$ lasers. Of additional interest, it may be possible to provide pre-heating of the accelerated pellet via the same laser pulse, which can significantly increase the final temperature achieved during pellet compression. For these reasons, the laser ablation concept was chosen for a more detailed initial evaluation.

Following the model of Jarboe [25] and Jarboe et al. [26], the laser ablation concept is treated using the rocket equation, where the ablated material plays the role of reaction mass expelled from the system:

$$
\Delta \mathrm{v}=\mathrm{v}_{\mathrm{e}} \ln \left(\mathrm{m}_{0} / \mathrm{m}_{\mathrm{f}}\right)
$$

where $\Delta \mathrm{v}$ is the velocity imparted to the pellet, $\mathrm{v}_{\mathrm{e}}$ is the velocity of the ablated material leaving the pellet, $\mathrm{m}_{0}$ is the initial pellet mass, and $\mathrm{m}_{\mathrm{f}}$ is the final pellet mass following ablation. For the gradient field concept the required pellet velocity is on the order of $10 \mathrm{~km} / \mathrm{s}$, hence the equation can be inverted to determine the fraction of material that must be ablated to provide this velocity:

$$
1-\mathrm{m}_{\mathrm{f}} / \mathrm{m}_{0}=1-\exp \left(-\Delta \mathrm{v} / \mathrm{f} \cdot \mathrm{v}_{\mathrm{e}}\right)
$$

Here an "exhaust" shape factor (f) has been included in the expression to account for ablated material leaving the surface at angles to the axial direction of motion; laser ablation experiments performed on conically shaped pellets provide a shape factor of approximately 0.6 [24].

Assuming that the energy imparted to the ablated material is converted to kinetic energy of the ions, the velocity $\mathrm{v}_{\mathrm{e}}$ is given by [26]:

$$
\mathrm{v}_{\mathrm{e}}=\left(\frac{4 \mathrm{I}}{\mathrm{n}_{\mathrm{c}} \mathrm{m}_{\mathrm{i}}}\right)
$$

where $\mathrm{I}$ is the laser intensity illuminating the pellet, $\mathrm{n}_{\mathrm{c}}$ is the critical density at which the incident laser light frequency equals the plasma frequency, and $\mathrm{m}_{\mathrm{i}}$ is the mass of the ion species being accelerated.

The critical density is given by:

$$
\mathrm{n}_{\mathrm{c}}=\left(\frac{\mathrm{m}_{\mathrm{e}} \varepsilon_{0} \omega^{2}}{\mathrm{e}^{2}}\right)
$$

where $\mathrm{m}_{\mathrm{e}}$ is the electron mass, $\varepsilon_{0}$ is permittivity of free space, $\omega$ is the frequency of the incident laser light, and e is the elementary unit of charge. Assuming a $\mathrm{CO}_{2}$ laser with a wavelength of $10.6 \mu \mathrm{m}$, the laser light frequency is $2.83 \times 10^{13} / \mathrm{s}$. Substituting into the equation above yields a critical density of $2.52 \times 10^{23} / \mathrm{m}^{3}$.

Assuming the laser illuminates and ablates liner material, a choice must be made for the liner. As noted in the preceding section, various liner materials have been proposed for MIF pellets, including aluminum, lithium and beryllium. For this sample calculation, aluminum is chosen as a representative liner material. The ion mass is then given by $\mathrm{m}_{\mathrm{i}}=27 \cdot\left(1.6726 \times 10^{-27}\right) \mathrm{kg}=4.52 \times 10^{-26} \mathrm{~kg}$.

The laser illumination can be estimated using available laser powers and appropriately sized beam widths that approximate the pellet cross section. Available high power CO2 lasers can readily provide 10-kW of power; assuming an initial pellet radius of 1-cm provides a cross sectional areas of around $3.14 \times 10^{-4} \mathrm{~m}^{2}$, for a pulsed laser intensity of $3.18 \times 10^{7} \mathrm{~W} / \mathrm{m}^{2}$.

Combining these results into a calculation of ablated ion velocity yields: 


$$
\mathrm{v}_{\mathrm{e}}=\left(\frac{(4)\left(3.18 \times 10^{7}\right)}{\left(2.52 \times 10^{23}\right)\left(4.52 \times 10^{-26}\right)}\right)^{1 / 2}=1.06 \times 10^{5} \frac{\mathrm{m}}{\mathrm{s}}
$$

The fraction of ablated liner mass required to provide this velocity is then given by:

$$
1-\mathrm{m}_{\mathrm{f}} / \mathrm{m}_{0}=1-\exp \left(-10^{4} /(0.6)\left(1.06 \times 10^{5}\right)\right)=0.15
$$

Thus, for the given parameters, ablating 15\% of the liner mass would provide a pellet injection velocity of $10 \mathrm{~km} / \mathrm{s}$. Increasing the incident laser energy on the target would increase the ablated ion velocity and reduce the amount of ablated material required to reach the desired injection velocity. For example, increasing the laser power from 10 $\mathrm{kW}$ to $100 \mathrm{~kW}$ in Equation 7 would increase the ion velocity to $3.33 \times 10^{5} \mathrm{~m} / \mathrm{s}$; substituting into Equation 8 indicates that approximately $5 \%$ of the initial target (liner) material would need to be ablated at this higher power level to provide the required $10 \mathrm{~km} / \mathrm{s}$ injection velocity. A $1 \mathrm{MW}$ pulse reduces the mass requirement even further to around $1.5 \%$. This mass could be accounted for by adding additional mass to the upstream face of the pellet, designed to be ablated away by the pulsed laser illumination. Alternatively, it may be possible to design the pellet with different ablative material at the back of the pellet, or if DT ice is used as the fuel, illuminate the ice directly to provide a lower ablative ion mass and higher exhaust velocity. In addition, once the required velocity is imparted to the pellet and the ablative mass is worn away, the laser pulse may be tailored to continue for a brief period to heat the internal fuel as the target enters the magnetic field and begins to compress. Achieving an internal fuel temperature of several tens to hundreds of $\mathrm{eV}$ prior to compression would reduce the amount of compression required to achieve fusion temperatures, which in turn can reduce the demands on pellet acceleration and compressive field strengths. Laser heating of D-T fuel pellets is an active area of investigation in magnetized liner inertial fusion; Sandia National Laboratories incorporates a $2.5 \mathrm{~kJ}, 1 \mathrm{TW}$ Nd:YAG laser in their z-pinch experiments to preheat a stationary target prior to compression. ${ }^{18}$

\section{Magnetic Field Coil Design}

A simple solenoid magnetic field coil is assumed for this preliminary analysis. Steady-state super-conducting solenoid coils with field strengths up to 30-T are now available [27], and it is reasonable to expect coils at or above this field strength can be designed and manufactured for the proposed concept. As concept definition progresses, more complex coil geometries can be incorporated, including magnetic nozzle coils and transition coils between the primary gradient field coil and the magnetic nozzle. However, to investigate whether the concept can compress and heat a rapidly injected target within a reasonable distance and with realistic field strengths, a simple solenoid coil model will be used. Because typical target diameters are small (cm-scale or less), a small-bore magnet can be used which should help reduce system mass and volume. Using a simple solenoid model also allows a ready calculation of axial and radial magnetic field strengths and gradients for use in the analytic and more advanced numerical models discussed below.

Some initial constraints on the axial length of the field coil can be estimated by assuming the injected target must be compressed and burned before the external (driving) magnetic field diffuses into the target, and exhausted into a magnetic nozzle before it dissociates to prevent damage to the coil. The diffusion time $(\tau)$ for a magnetic field to penetrate through a conducting layer into a cylindrical target can be estimated as:

$$
\tau \approx \frac{\mu \sigma \Delta r}{2}
$$

where $\mu, \sigma$, and $\Delta$ are the permeability, conductivity, and thickness of the liner, and $\mathrm{r}$ is the radius. For typical liner materials the diffusion time is roughly on the order of $10^{-3} \mathrm{~s}$. For an assumed initial target velocity of around 10$\mathrm{km} / \mathrm{s}$, the target will travel a distance of approximately $10 \mathrm{~m}$ before the external magnetic field will diffuse into the interior of the target. A more stringent constraint is associated with the compression and burn time of the target. For the same initial target velocity and a compression time on the order of $10-\mu \mathrm{s}$, the distance travelled by the target (assuming no slowing down within the coil) is around $10 \mathrm{~cm}$, indicating that the coil needs to be sufficiently short to allow the target to compress and burn within this distance before expanding into the nozzle region. While more detailed estimates of coil length can be derived from the compression models, the initial analysis indicates the magnetic field coil will likely be less than a meter in length.

Once fusion is achieved and the plasma begins to expand, it must be directed into axial thrust. The initial assumption is that a magnetic nozzle will be used for this purpose, and a numerical model has been developed by the University of Alabama Huntsville (UAH) to simulate this effect [28]. In this model the coil windings are grouped in stages, with the number of windings and current in each stage treated as variables. The field is highest at the throat 
and expands downstream. The axial field gradient can be increased by increasing the number of windings, the current, or both, at the throat section while decreasing the field on either side of the throat stage. An example of a simulated magnetic nozzle is presented in Fig. 5. Once the numerical modeling work underpinning the compression and burn physics is completed, the model will be integrated with the magnetic nozzle simulation to provide a complete system description. More immediately, a magnetic nozzle efficiency factor can be employed to estimate system performance as discussed in the section below.

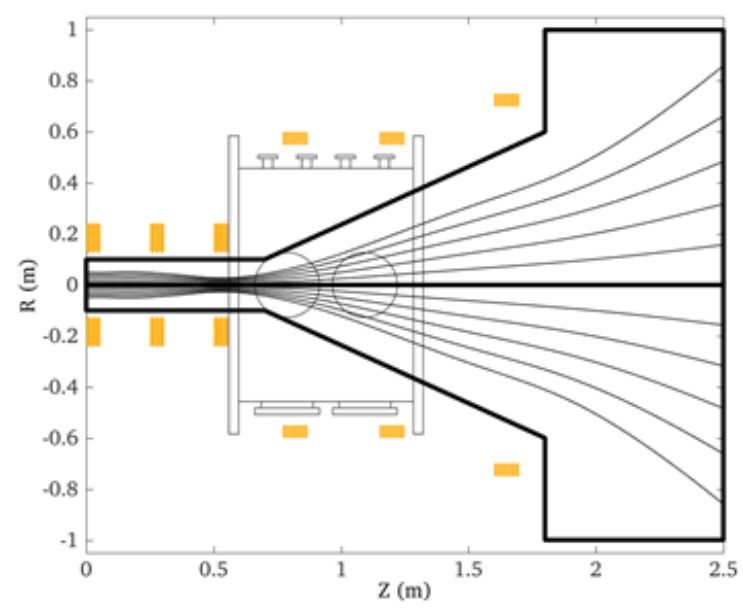

Fig. 5 Example of magnetic field topology (stream lines) generated by coaxial windings of magnetic field coils (yellow). Outline of representative nozzle walls and chamber geometry are for illustrative purposes [28].

\section{Estimating System Performance}

The analytic and numerical models developed in this study provide an estimate of the fusion yield energy, which in turn can be used to roughly estimate the exhaust velocity of the plasma propellant. Equating the yield energy, E, to the directed kinetic energy of a propellant provides the following equation for the exhaust velocity, $\mathrm{v}_{\mathrm{e}}$ :

$$
\mathrm{v}_{\mathrm{e}}=\mathrm{v}_{\mathrm{z}_{\mathrm{t}} \text { target }}+\left(\frac{2 \eta \mathrm{E}}{\mathrm{m}}\right)^{1 / 2}
$$

where $\mathrm{v}_{\mathrm{z}_{\_} \text {target }}$ is the axial velocity of the target imparted by the accelerator (taking into account any reduction ${ }^{6}$ due to traversing the radial portion of the magnetic field gradient), $m$ is the expelled propellant mass, and $\eta$ is the efficiency at which the magnetic nozzle converts the uniformly expanding plasma into directed axial thrust. Values for $\eta$ can vary widely, and the performance of the system will be more accurately represented through an integrated numerical simulation of the compression, burn, and expansion of the fusion plasma in the magnetic nozzle. However, for these preliminary estimates we assume a value of $\eta \approx 0.7$, consistent with recommended practice [29].

The specific impulse $\left(\mathrm{I}_{\mathrm{sp}}\right)$ of the system can then be estimated using:

$$
\mathrm{I}_{\mathrm{sp}}=\mathrm{v}_{\mathrm{e}} / \mathrm{g}_{0}
$$

where $g_{0}$ is the acceleration due to gravity $\left(9.8 \mathrm{~m} / \mathrm{s}^{2}\right)$. The impulse ( $\left.\mathrm{I}_{\mathrm{bit}}\right)$ provided by the pulsed exhaust is calculated by:

$$
\mathrm{I}_{\mathrm{bit}}=\mathrm{mv}_{\mathrm{e}}=\frac{\mathrm{mI}_{\mathrm{sp}}}{\mathrm{g}_{0}}(\mathrm{~N}-\mathrm{s})
$$

For a pulse repetition frequency $\mathrm{f}(\mathrm{Hz})$, the average thrust, $\mathrm{F}_{\mathrm{av}}$, provided by the system is given by:

$$
\mathrm{F}_{\mathrm{av}}=\mathrm{f}(\mathrm{Hz}) \cdot \mathrm{I}_{\mathrm{bit}}
$$

Once the yield energy is defined, these equations can be used to estimate the performance of the system.

\footnotetext{
${ }^{6}$ The analytic model described in Section III was used to determine the change in initial target velocity caused by radial magnetic field pressure within the magnetic field gradient; in general, the axial target velocity was reduced by less than $10 \%$.
} 
The overall gain of the fusion system can be estimated using the ratio of the yield energy to the energy required to accelerate and preheat the target (driver energy). As with other systems, using a laser will incur inefficiencies in converting the input power to laser power, and in converting the laser energy illuminating the target to directed kinetic energy. For an input power $\left(\mathrm{P}_{\text {in }}\right)$ to laser power conversion efficiency $\eta_{\text {in }}$ and a laser power to power on target $\left(P_{\text {target }}\right)$ conversion efficiency $\eta_{\text {target }}$, the gain $(\mathrm{G})$ may be defined as:

$$
\mathrm{G}=\frac{\mathrm{P}_{\text {fusion }}}{\left(\mathrm{P}_{\text {in }} / \eta_{\text {in }}\right)+\left(\mathrm{P}_{\text {target }} / \eta_{\text {target }}\right)}
$$

where $\mathrm{P}_{\text {fusion }}$ is the fusion power released by the imploding target. Gains exceeding unity are clearly desired for efficient system operation, but high gains can also place severe stress on vehicle structures. Defining the optimum gain to produce desired vehicle performance is integrally connected to the mission performance modeling described in a later section, and ties to requirements on specific impulse, power, and mass, leading to an iterative process between mission modeling and fusion system modeling.

\section{Semi-Analytic Compression Model}

A semi-analytic model was developed to help evaluate initial concept feasibility, and to provide initial approximations for magnetic field geometries, target fuel densities and initial velocities, target compression and heating, and fusion yields that could be used to guide more detailed numerical simulations and initial vehicle performance estimates. The model is based on the Semi-Analytic MagLIF Model (SAMM) developed by McBride and Slutz [30] for a terrestrial z-pinch geometry, modified and adapted to the current concept with additional simplifications to be discussed below. Given the unique nature of the concept under investigation, the quantitative values predicted by the modified semi-analytic model cannot be readily validated and provide crude approximations at best. However, the qualitative results likely predict correct trends and thus can be used to guide a smaller set of more accurate numerical simulations.

In its present form, the analytic model used in this study consists of several interlinked analytic expressions, encompassing fuel and magnetic field pressure terms, liner radial acceleration, optional fuel preheating, $\alpha$ particle energy deposition, radiative losses, ion and electron thermal conduction losses, fusion reaction rate calculations, energy balance, and gain calculations. Developed to simulate a z-pinch system, the original SAMM included circuit equations for axial current generation and corresponding induced azimuthal magnetic fields acting on a stationary DT fuel target. In the current model, a stationary magnetic field is assumed and a DT target is injected into the field with a user-defined velocity. For simplicity, the initial model assumes a linear magnetic field gradient up to a maximum value of magnetic field within the coil, after which the field is assumed to remain constant within the coil. This is fairly consistent with numerical simulations of solenoid coil magnetic fields and associated axial gradients, an example of which is shown in Fig. 6 below.
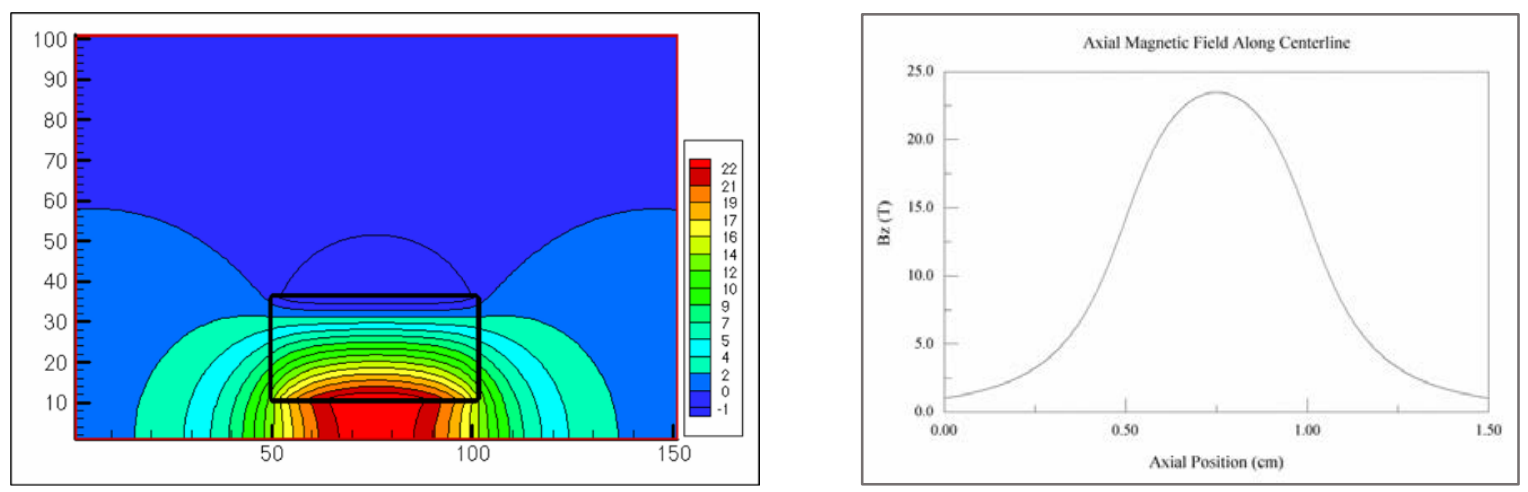

Fig. 6 Left: Example of half-plane contours for axial magnetic field strength (coil geometry in black box); Right: corresponding axial magnetic field strength along the magnet centerline as function of axial position.

User-defined inputs to the analytic model include the magnetic field coil radius, maximum axial magnetic field within the coil, axial magnetic field gradient, target outer radius and liner thickness, target length, liner material (currently aluminum, beryllium, or lithium), the initial target DT fuel mass density, initial target fuel temperature, initial target axial velocity, initial axial magnetic field internal to the target, and options to include target preheating, 
$\alpha$-heating, radiation losses, ion and electron radial thermal conduction losses, and magnetic nozzle efficiency. Time steps and total run times are also defined. With these inputs, the model runs through a series of calculations at each time step, following the flow described in the following sections.

\section{A. Liner Radial Acceleration}

For this initial simplified model it is assumed that the target liner is incompressible and remains at a constant thickness during compression. This is a gross simplification compared to the SAMM model, which treats the liner as multiple shells with corresponding material equations of state and dynamics to estimate liner compression during implosion. With a rigid liner the simplified model will likely overestimate the amount of target compression and the corresponding energy release compared to the more realistic SAMM liner model. While a compressible shell model is being considered for a later version of the current model, the initial simplification of a rigid liner provides a useful starting point for analyzing performance trends.

Ignoring internal liner shell pressures, the radial acceleration of the fuel-liner interface $\left(\mathrm{r}_{\mathrm{fl}}\right)$ is given by:

$$
\ddot{\mathrm{r}}_{\mathrm{fl}}=\frac{\mathrm{P}_{\mathrm{f}}+\mathrm{P}_{\mathrm{B}_{\mathrm{i}}}-\mathrm{P}_{\mathrm{l}_{\mathrm{i}}}}{\mathrm{m}_{\mathrm{l}} / 2} \cdot 2 \pi \mathrm{r}_{\mathrm{f}} \mathrm{L}
$$

and that of the liner-vacuum interface $\left(\mathrm{r}_{\mathrm{lv}}\right)$ is given by:

$$
\ddot{\mathrm{r}}_{\mathrm{lv}}=\frac{\mathrm{P}_{\mathrm{I}_{\mathrm{v}}}-\mathrm{P}_{\mathrm{B}_{\mathrm{v}}}}{\mathrm{m}_{\mathrm{l}} / 2} \cdot 2 \pi \mathrm{r}_{\mathrm{l}} \mathrm{L}
$$

where $\mathrm{P}_{\mathrm{f}}$ is the isobaric (ideal gas) fuel pressure, $\mathrm{P}_{\mathrm{Bi}}$ is the magnetic field pressure internal to the target, $\mathrm{P}_{\mathrm{li}}$ is the liner pressure facing the fuel, $\mathrm{P}_{\mathrm{lv}}$ is the liner pressure facing the vacuum, $\mathrm{P}_{\mathrm{bv}}$ is the magnetic field pressure external to the target, $m_{l}$ is the liner mass, $r_{f}$ is the fuel radius, $r_{l}$ is the external target radius (including liner), and $L$ is the liner length. In this approximation, half the liner mass is attributed to the liner-fuel interface, and half to the linervacuum interface. For an incompressible liner, $\mathrm{P}_{\mathrm{li}}=\mathrm{P}_{\mathrm{lv}}$ and the radial acceleration of the fuel-liner interface is equal to the radial acceleration of the liner-vacuum interface. As such, Equations 15 and 16 can be combined to provide an expression for the radial acceleration of the target liner:

$$
\ddot{\mathrm{r}_{\mathrm{l}}}=\frac{2 \pi \mathrm{L}}{\mathrm{m}_{\mathrm{l}}}\left(\mathrm{P}_{\mathrm{f}}+\mathrm{P}_{\mathrm{B}_{\mathrm{i}}}-\mathrm{P}_{\mathrm{B}_{\mathrm{v}}}\right) \mathrm{r}_{\mathrm{l}}-\left(\mathrm{P}_{\mathrm{f}}+\mathrm{P}_{\mathrm{B}_{\mathrm{i}}}\right) \Delta
$$

where $\Delta$ is the thickness of the (incompressible) liner. Pressure values are calculated for each time step, and the radial acceleration is updated. From this updated acceleration, the liner radial velocity and radial position can then be determined at each step.

\section{B. Pressure Terms}

1) The isobaric fuel pressure is given by $\mathrm{P}_{\mathrm{f}}=\rho \mathrm{RT}$, where $\rho$ is the fuel mass density, $\mathrm{R}$ is the universal gas constant, and $\mathrm{T}$ is the fuel temperature.

2) Magnetic field pressure internal to target:

$$
\mathrm{P}_{\mathrm{B}_{\mathrm{i}}}=\frac{\mathrm{B}_{\mathrm{i}}^{2}}{2 \mu_{0}}
$$

Here, $B_{i}$ is the internal magnetic field strength, and $\mu_{0}$ is the permeability of free space. During target compression the internal magnetic field increases as the initially trapped magnetic flux is compressed into an ever shrinking radius. For an initial trapped magnetic field $B_{i 0}$ and target radius $r_{0}$, the magnetic flux $\varphi_{i}$ confined within the uncompressed target is:

$$
\varphi_{\mathrm{i}}=\mathrm{B}_{\mathrm{i} 0}\left(\pi \mathrm{r}_{0}^{2}\right)
$$

Assuming the magnetic flux remains constant during compression (no flux leakage), the magnetic field $\mathrm{B}_{\mathrm{i}}$ at target radius $r$ is then:

$$
\mathrm{B}_{\mathrm{i}}=\frac{\mathrm{B}_{\mathrm{i} 0}\left(\pi \mathrm{r}_{0}^{2}\right)}{\pi \mathrm{r}^{2}}=\mathrm{B}_{\mathrm{i} 0}\left(\frac{\mathrm{r}_{0}}{\mathrm{r}}\right)^{2}
$$


For even modest compression ratios $\left(\mathrm{r}_{\mathrm{o}} / \mathrm{r}\right)$, an initially trapped magnetic field of a few to several Tesla within the target can significantly increase to axial field values sufficient for trapping fusion $\alpha$ particles and suppressing the radial thermal conduction of ions and electrons.

3) Magnetic field pressure external to target:

$$
\mathrm{P}_{\mathrm{B}_{\mathrm{v}}}=\frac{\mathrm{B}_{\mathrm{v}}^{2}}{2 \mu_{0}}
$$

For the more simple initial version of the code developed here, the vacuum magnetic field is assumed to consist of a gradient region followed by a maximum constant axial field within the coil. As noted in a prior section, the axial gradient is assumed to be linear to simplify the analytic model. It is assumed that any magnetic flux external to the target does not penetrate the liner during compression (compression time $<<$ field diffusion time). The flux within the solenoid coil prior to target entry is given by:

$$
\varphi_{\mathrm{v}}=\mathrm{B}_{\mathrm{v} 0}\left(\pi \mathrm{r}_{\mathrm{c}}^{2}\right)
$$

where $B_{v 0}$ is the initial vacuum magnetic field within the coil and $r_{c}$ is the solenoid coil radius. As the target enters the coil the flux will be compressed between the target liner and the internal coil wall:

$$
\varphi_{\mathrm{v}}=\mathrm{B}_{\mathrm{v}} \pi\left(\mathrm{r}_{\mathrm{c}}^{2}-\mathrm{r}_{1}^{2}\right)
$$

For conserved flux, the displaced magnetic field external to the liner as the target traverses the coil is then given by:

$$
\mathrm{B}_{\mathrm{v}}=\frac{\mathrm{B}_{\mathrm{v} 0}\left(\mathrm{r}_{\mathrm{c}}^{2}\right)}{\left(\mathrm{r}_{\mathrm{c}}^{2}-\mathrm{r}_{\mathrm{l}}^{2}\right)}
$$

For an initial target radius close to the inner coil radius, the compressed flux can significantly increase the magnetic field strength between the coil and target, in turn providing enhanced magnetic field pressure to compress the target.

\section{Energy Terms}

The total fuel energy changes during each time step due to multiple heating and cooling terms, which are described in detail in McBride and Slutz [30] and summarized below.

1) Adiabatic Heating $\left(\dot{\mathrm{E}}_{\mathrm{ad}}\right)$ : The adiabatic heating rate is given by $\dot{\mathrm{E}}_{\mathrm{ad}}=\mathrm{P}_{\mathrm{f}} \dot{\mathrm{V}}_{\mathrm{f}}$, where $\mathrm{P}_{\mathrm{f}}$ is the internal isobaric fuel pressure (defined above) and $\dot{\mathrm{V}}_{\mathrm{f}}$ is the change in fuel volume calculated at each time step.

2) Fuel Preheating $\left(\dot{\mathrm{E}}_{\mathrm{pre}}\right)$ : As noted, several ground based experiments and simulations have demonstrated significant improvements in final compressed fuel temperatures if the fuel is preheated prior to compression. In most cases this is accomplished via pulsed, high intensity laser illumination of the target to raise the fuel temperature several 10's to 100's of eV just prior to implosion. The SAMM code [30] includes laser preheating through a target window, simulating recent Sandia experiments. For this more simple initial model, the analysis includes an option to ramp the fuel temperature from an initial temperature to a user defined temperature over a given time period. The time period can be adjusted to provide a temperature change before the target enters the gradient region, or during traversal of the region prior to the start of radial compression. While crude, it offers at least an initial ability to investigate the effects of preheated fuel temperatures on the performance of the concept.

3) $\alpha$-Particle Heating $\left(\dot{\mathrm{E}}_{\alpha}\right)$ : If the axial magnetic field internal to the target is sufficiently strong at compression, the $\alpha$ particles released during D-T fusion will be trapped and deposit some fraction of their energy into the fuel. For a given $\alpha$ particle energy $\mathrm{Q}_{\alpha}$, the fraction $\mathrm{f}_{\alpha}$ of energy deposited in the fuel is given by [30]:

$$
f_{\alpha}=\frac{x_{\alpha}+x_{\alpha}^{2}}{1+\frac{13 x_{\alpha}}{9}+x_{\alpha}^{2}}
$$

with

$$
\mathrm{x}_{\alpha}=\frac{8}{3}\left(\frac{\mathrm{r}_{\mathrm{f}}}{\mathrm{l}_{\alpha}}+\frac{\mathrm{b}^{2}}{\sqrt{9 \mathrm{~b}^{2}+1000}}\right)
$$

and

$$
\mathrm{l}_{\alpha}=\left(4 \pi \epsilon_{0}\right)^{2} \cdot \frac{3}{4 \sqrt{2 \pi}} \cdot \frac{\mathrm{m}_{\alpha} \mathrm{v}_{\alpha 0}\left(\mathrm{kT}_{\mathrm{f}}\right)^{3 / 2}}{\overline{\mathrm{n}_{\mathrm{e}}} \mathrm{Z}_{\alpha}^{2} \mathrm{q}_{\mathrm{e}}^{4} \mathrm{~m}_{\mathrm{e}}^{1 / 2} \ln \Lambda}
$$




$$
\begin{gathered}
b=\frac{r_{f}}{r_{\alpha L}} \\
r_{\alpha L}=\frac{m_{\alpha} v_{\alpha 0}}{Z_{\alpha} q_{e} B_{i}}
\end{gathered}
$$

In the above, $\mathrm{l}_{\alpha}$ is the mean free path of an $\alpha$ particle, $\mathrm{m}_{\alpha}$ is the $\alpha$ particle rest mass, $\mathrm{v}_{\alpha 0}$ is the initial velocity of the $\alpha$ particle $\left(=\sqrt{2 Q_{\alpha} / m_{\alpha}}\right.$, where $Q_{\alpha}$ is the $\alpha$ particle energy), $\mathrm{Z}_{\alpha}$ is the $\alpha$ charge $(=2), \mathrm{r}_{\alpha \mathrm{L}}$ is the $\alpha$ particle Larmor radius, and $\ln (\Lambda)$ is the Coulomb logarithm. Using these equations, the heating rate due to $\alpha$ particle energy deposition is then given by:

$$
\dot{\mathrm{E}}_{\alpha}=\dot{\mathrm{N}}_{\mathrm{DT}} \mathrm{Q}_{\alpha} \mathrm{f}_{\alpha}
$$

where $\dot{\mathrm{N}}_{\mathrm{DT}}$ is the DT reaction rate, discussed in a later section.

4) Radiative Cooling: The initial radiative cooling term included in this simplified analytic model was limited to bremsstrahlung radiation, given by:

$$
\dot{\mathrm{E}}_{\mathrm{r}}=1.57 \times 10^{-40} \overline{\mathrm{Z}}_{\mathrm{f}} \overline{\mathrm{n}}_{\mathrm{i}} \overline{\mathrm{n}}_{\mathrm{e}} \sqrt{\overline{\mathrm{T}}_{\mathrm{f}}} \cdot\left(\pi \mathrm{r}_{\mathrm{f}}^{2} \mathrm{~L}\right)
$$

which assumes a constant density and temperature over the entire fuel volume, and an optically thin volume emitter. This radiative model significantly overestimated the fuel cooling rates, and in many cases overwhelmed the heating terms to prevent any temperature rise in the plasma. While circumstances can exist where radiative cooling can exceed adiabatic and other heating sources, the rapid radiative energy loss observed in several cases for the initial analytic model was somewhat suspect, leading to the need for a more accurate approach. As such, the gray radiation model discussed in McBride and Slutz [30] seemed a more suitable representation. The gray model incorporates two-temperature effects as well as liner radiation absorption and re-emission. Due to the limited time available for the study, a simplified version of the gray model was adapted for the current analysis. In this adaptation, the volume radiation $\left(\mathrm{P}_{\mathrm{rv}}\right)$ is set equal to the gray body surface radiation $\left(\mathrm{P}_{\mathrm{rs}}\right)$, with the terms defined as:

$$
\begin{gathered}
P_{r v}=A_{b r} \cdot 2 \pi L \cdot \bar{Z}_{f} \int_{0}^{r_{f}} n_{i} n_{e} \sqrt{T_{f}}\left[1-\left(\frac{T_{B}}{T_{f}}\right)^{4}\right] r d r \\
P_{r s}=\left(1-\alpha_{s}\right) \sigma T_{B}^{4} \cdot 2 \pi r_{f} L
\end{gathered}
$$

where $A_{b r}=1.57 \times 10^{-40} \mathrm{~m}^{3} \mathrm{~K}^{-1 / 2} \mathrm{~J} / \mathrm{s}$, $\mathrm{L}$ is the length of the cylindrical fuel element, $\bar{Z}_{\mathrm{f}}$ is the average ionization state of the fuel, $\sigma$ is the Stephan-Boltzmann constant, $T_{\mathrm{f}}$ is the fuel temperature, $T_{\mathrm{B}}$ represents the brightness temperature, and $\alpha_{\mathrm{s}}$ is the albedo of the liner's inner surface (typically $\geq 0.9$ ). In the simple analytic model used here, the albedo is set to 0.9 , and number densities and temperatures are assumed uniform over the fuel volume. With these approximations, the equations are set equal and the brightness temperature, $\mathrm{T}_{\mathrm{B}}$, is solved and used to determine the total radiated power at each time step.

5) Thermal Conduction Cooling $\left(\dot{\mathrm{E}}_{\mathrm{tc}}\right)$ : The SAMM code [30] differentiates fuel regions into a high temperature central hot spot and cooler shelf region, which provides a well-defined radial thermal gradient for thermal conduction to occur. The simplified model used here assumes a uniform volumetric fuel density and temperature, and uses an assumed linear radial thermal profile to approximate potential thermal conduction losses. In this instance, the energy loss rate due to electron thermal conduction is given by [30]:

$$
\dot{\mathrm{E}}_{\mathrm{tc}, \mathrm{e}}=2 \pi \mathrm{rL} \cdot \kappa_{\mathrm{e}} \cdot \mathrm{k} \frac{\partial \mathrm{T}_{\mathrm{f}}}{\partial \mathrm{r}} \approx 2 \pi \mathrm{L} \cdot \kappa_{\mathrm{e}} \cdot \mathrm{k} \frac{\mathrm{T}_{\mathrm{f}}}{\mathrm{r}} \approx 2 \pi \mathrm{L} \cdot \kappa_{\mathrm{e}} \cdot \mathrm{kT}_{\mathrm{f}}
$$

where $\mathrm{k}$ is Boltzmann's constant, $\mathrm{L}$ is the target length, and $\kappa_{\mathrm{e}}$ is the electron thermal conduction coefficient perpendicular to the axial magnetic field internal to the target: 


$$
\kappa_{e}=\frac{n_{e} \mathrm{kT}_{\mathrm{f}} \tau_{\mathrm{ei}}}{\mathrm{m}_{\mathrm{e}}} \cdot \frac{6.18+4.66 \mathrm{x}_{\mathrm{e}}}{1.93+2.31 \mathrm{x}_{\mathrm{e}}+5.35 \mathrm{x}_{\mathrm{e}}^{2}+\mathrm{x}_{\mathrm{e}}^{3}}
$$

Here $n_{e}$ is the electron number density, $m_{e}$ is the electron rest mass, $x_{e}$ is the electron Hall parameter, defined as $\mathrm{X}_{\mathrm{e}} \equiv \omega_{\mathrm{ce}} \tau_{\mathrm{e}}$, where $\omega_{\mathrm{ce}}$ is the electron cyclotron frequency and $\tau_{\mathrm{ei}}$ is the average electron-ion collision time. Similarly for ions, the energy loss rate due to ion thermal conduction is given by [30]:

$$
\dot{\mathrm{E}}_{\mathrm{tc}, \mathrm{i}}=2 \pi \mathrm{rL} \cdot \kappa_{\mathrm{i}} \cdot \mathrm{k} \frac{\partial \mathrm{T}_{\mathrm{f}}}{\partial \mathrm{r}} \approx 2 \pi \mathrm{L} \cdot \kappa_{\mathrm{i}} \cdot \mathrm{kT}_{\mathrm{f}}
$$

with

$$
\kappa_{\mathrm{i}}=\frac{\mathrm{n}_{\mathrm{i}} \mathrm{kT}_{\mathrm{f}} \tau_{\mathrm{ii}}}{\overline{\mathrm{m}}_{\mathrm{i}}} \cdot \frac{2.645+2 \mathrm{x}_{\mathrm{i}}^{2}}{0.677+2.70 \mathrm{x}_{\mathrm{i}}^{2}+\mathrm{x}_{\mathrm{i}}^{4}}
$$

where $n_{\mathrm{i}}$ is the ion number density, $\overline{\mathrm{m}}_{\mathrm{i}}$ is the average ion mass, $\mathrm{x}_{\mathrm{i}}$ is the ion Hall parameter, defined as $\mathrm{x}_{\mathrm{i}} \equiv \omega_{\mathrm{ci}} \tau_{\mathrm{ii}}, \omega_{\mathrm{ci}}$ is the ion cyclotron frequency, and $\tau_{\mathrm{ii}}$ is the average ion-ion collision time. The total thermal conduction loss term is then given by:

$$
\dot{\mathrm{E}}_{\mathrm{tc}}=\dot{\mathrm{E}}_{\mathrm{tc}, \mathrm{e}}+\dot{\mathrm{E}}_{\mathrm{tc}, \mathrm{i}}
$$

6) End Losses $\left(\dot{\mathrm{E}}_{\text {end }}\right)$ : Particles escaping from the ends of the target during compression constitute an additional energy loss term, as well as contributing to the loss of fuel mass. The expression for particle end losses is modeled after [30]:

$$
\dot{\mathrm{E}}_{\text {end }}=2 \cdot\left(\frac{3}{4}\right)^{4} \frac{\mathrm{E}_{\mathrm{f}}}{\mathrm{V}_{\mathrm{f}}} \int_{0}^{\mathrm{r}_{\mathrm{f}}} \mathrm{c}_{\mathrm{f}} \cdot 2 \pi \mathrm{r} \cdot \mathrm{dr}=2 \cdot\left(\frac{3}{4}\right)^{4} \frac{\mathrm{E}_{\mathrm{f}}}{\mathrm{V}_{\mathrm{f}}} \mathrm{c}_{\mathrm{f}} \cdot\left(\pi \mathrm{r}_{\mathrm{f}}^{2}\right)
$$

where

$$
\mathrm{c}_{\mathrm{f}}=\left(\gamma_{\mathrm{f}} \mathrm{p}_{\mathrm{f}} / \rho_{\mathrm{f}}\right)^{1 / 2}
$$

is the speed of sound in the fuel region and $\gamma_{\mathrm{f}}=5 / 3$ is the ratio of specific heats for an ideal gas. For this initial evaluation, it is assumed that particle losses are identical from each end of the target (yielding the factor of 2 in Equation 39) and that the fuel pressure and density are uniform throughout the fuel region. In addition to energy loss, the loss of particles from the ends of the target also creates a mass loss, which is further discussed in Section III.E.

\section{Net Energy Change and Fuel Temperature}

The combined effect $\left(\dot{E}_{\mathrm{f}}\right)$ of the energy gain and loss terms is given by the expression:

$$
\dot{\mathrm{E}}_{\mathrm{f}}=\dot{\mathrm{E}}_{\mathrm{ad}}+\dot{\mathrm{E}}_{\mathrm{pre}}+\dot{\mathrm{E}}_{\alpha}-\dot{\mathrm{E}}_{\mathrm{r}}-\dot{\mathrm{E}}_{\mathrm{tc}}-\dot{\mathrm{E}}_{\mathrm{end}}
$$

which is evaluated at each time step to determine the net change in fuel energy. Multiplying $\dot{E}_{\mathrm{f}}$ by the time step $\Delta t$ yields the net energy change in the fuel for each time step. The cumulative energy in the fuel, $\mathrm{E}_{\mathrm{f}}$, is then calculated, and from that total energy an average fuel temperature can be determined:

$$
\mathrm{T}_{\mathrm{f}}=\frac{2 \mathrm{E}_{\mathrm{f}}}{3 \mathrm{k}\left(\mathrm{N}_{\mathrm{D}}+\mathrm{N}_{\mathrm{T}}+\mathrm{N}_{\mathrm{e}}\right)}
$$

where $\mathrm{N}_{\mathrm{D}}, \mathrm{N}_{\mathrm{T}}$, and $\mathrm{N}_{\mathrm{e}}$ are the total number of deuterons, tritons, and electrons in the fuel. The initial numbers and number densities of $\mathrm{D}, \mathrm{T}$ are determined from the initial user-defined fuel density and volume, which provides the fuel mass, $\mathrm{M}_{\mathrm{f}}$. For this initial model, a 50:50 fuel mixture of $\mathrm{D}$ and $\mathrm{T}$ is assumed. For this approximation, the number of $\mathrm{D}$ and $\mathrm{T}$ atoms in the fuel is given by:

$$
\mathrm{N}_{\mathrm{D}}=\mathrm{N}_{\mathrm{T}}=\frac{\mathrm{M}_{\mathrm{f}}}{\left(\mathrm{m}_{\mathrm{D}}+\mathrm{m}_{\mathrm{T}}\right)}
$$


where $\mathrm{m}_{\mathrm{D}}$ and $\mathrm{m}_{\mathrm{T}}$ are the deuteron and triton masses, respectively. The fuel mass will change as a result of fusion events (fuel burnup), and $\mathrm{N}_{\mathrm{D}}$ and $\mathrm{N}_{\mathrm{T}}$ will change in equal proportion as each are consumed in the DT fusion reactions. The rate of change for $\mathrm{N}_{\mathrm{D}}$ and $\mathrm{N}_{\mathrm{T}}$ are governed by fusion reaction rates, discussed below. The number of electrons is assumed to be given by $\mathrm{N}_{\mathrm{e}}=\mathrm{N}_{\mathrm{D}}+\mathrm{N}_{\mathrm{T}}$; as the fuel temperature rises above several $\mathrm{eV}$, the fuel becomes nearly fully ionized and each atom gives up one electron. A more detailed ionization model is being evaluated for inclusion in the model, but for this initial analysis the approximation of full ionization due either to laser preheating or to adiabatic heating shortly after the start of target compression appears justified based on an initial Saha ionization analysis. The number of each species divided by the fuel volume provides the number density ( $\mathrm{n}_{\mathrm{s}}$ ) for each species, which will change as the fuel volume changes during compression and as D and T ions are consumed by fusion reactions.

\section{E. Fusion Reaction Rates}

Consistent with the SAMM model, the DT reaction rate used in the simplified model is:

$$
\dot{\mathrm{N}}_{\mathrm{DT}}=\mathrm{L} \int_{0}^{\mathrm{r}_{\mathrm{f}}} \mathrm{n}_{\mathrm{D}} \mathrm{n}_{\mathrm{T}}\langle\sigma v\rangle_{\mathrm{DT}} \cdot 2 \pi \mathrm{r} \cdot \mathrm{dr}
$$

where $n_{D}$ and $n_{T}$ are the fuel deuteron and triton number densities, respectively, and $\langle\sigma v\rangle_{D T}$ is the reactivity parameter for DT reactions. For DT the reactivity parameter is calculated as [30]:

$$
\begin{gathered}
\langle\sigma \mathrm{v}\rangle_{\mathrm{DT}}=\mathrm{C}_{1} \zeta^{-5 / 6} \xi^{2} \exp \left(-3 \zeta^{1 / 3} \xi\right) \\
\zeta=1-\frac{\mathrm{C}_{2} \mathrm{~T}_{\mathrm{f}, \mathrm{keV}}+\mathrm{C}_{4} \mathrm{~T}_{\mathrm{f}, \mathrm{keV}}^{2}+\mathrm{C}_{6} \mathrm{~T}_{\mathrm{f}, \mathrm{keV}}^{3}}{1+\mathrm{C}_{3} \mathrm{~T}_{\mathrm{f}, \mathrm{keV}}+\mathrm{C}_{5} \mathrm{~T}_{\mathrm{f}, \mathrm{keV}}^{2}+\mathrm{C}_{7} \mathrm{~T}_{\mathrm{f}, \mathrm{keV}}^{3}} \\
\xi=\frac{\mathrm{C}_{0}}{\mathrm{~T}_{\mathrm{f}, \mathrm{keV}}^{1 / 3}}
\end{gathered}
$$

where $T_{f, k e V}$ is the fuel temperature expressed in units of $\mathrm{keV}$, and $\mathrm{C}_{0}-\mathrm{C}_{7}$ are fitting parameters used in SAMM and reproduced below for DT fuel:

Table 1. Coefficients for reaction rate calculations. Adapted from [30].

\begin{tabular}{|c|c|}
\hline Coefficient & DT Fuel \\
\hline $\mathrm{Co}$ & 6.6610 \\
\hline $\mathrm{C} 1 \times 10^{22}$ & 630.5 \\
\hline $\mathrm{C} 2 \times 10^{3}$ & 15.136 \\
\hline $\mathrm{C} 3 \times 10^{3}$ & 75.189 \\
\hline $\mathrm{C} 4 \times 10^{3}$ & 4.6064 \\
\hline $\mathrm{C} 5 \times 10^{3}$ & 13.500 \\
\hline $\mathrm{C} 6 \times 10^{3}$ & -0.10675 \\
\hline $\mathrm{C} 7 \times 10^{3}$ & 0.01366 \\
\hline
\end{tabular}

For the model presented here, the number density and temperature of the DT fuel is assumed to be uniform, which simplifies the reaction rate to $\dot{\mathrm{N}}_{\mathrm{DT} \_ \text {fus }}=\mathrm{n}_{\mathrm{D}} \mathrm{n}_{\mathrm{T}}\langle\sigma \mathrm{v}\rangle_{\mathrm{DT}} \pi \mathrm{r}_{\mathrm{f}}^{2} \mathrm{~L}$. The rate at which the numbers of $\mathrm{D}$ and $\mathrm{T}$ ions are reduced due to fusion reactions is then given by:

$$
\dot{\mathrm{N}}_{D_{-} \text {fus }}=\dot{\mathrm{N}}_{\mathrm{T}_{f} \text { fus }}=-\dot{\mathrm{N}}_{\mathrm{DT} \_ \text {fus }}
$$

Ignoring for now any secondary reactions, the rate at which deuterons and tritons are lost to D-T fusion reactions is also the rate at which neutrons are produced (one $14.1 \mathrm{MeV}$ neutron per reaction), allowing the neutron number to be tracked for later fluence calculations.

As previously noted, particles will also be lost from the ends of the target during compression and heating, constituting an additional change in ion number and number density within the fuel region. The rate at which particles are lost from each end of the cylindrical fuel region are assumed here to be equal, and given by [30]: 


$$
\dot{\mathrm{N}}_{\mathrm{D} \_ \text {ends }}=\dot{\mathrm{N}}_{\mathrm{T} \_ \text {ends }}=-2 \cdot\left(\frac{3}{4}\right)^{4} \int_{0}^{\mathrm{r}_{\mathrm{f}}} \mathrm{n}_{\mathrm{D}, \mathrm{T}} \cdot \mathrm{c}_{\mathrm{f}} \cdot 2 \pi \mathrm{r} \cdot \mathrm{dr}=2 \cdot\left(\frac{3}{4}\right)^{4} \mathrm{n}_{\mathrm{D}, \mathrm{T}} \cdot \mathrm{c}_{\mathrm{f}} \cdot\left(\pi \mathrm{r}_{\mathrm{f}}^{2}\right)
$$

where again the factor of 2 accounts for particle losses from both ends and uniform pressure and density are assumed throughout the fuel region.

The rate at which particles are lost from the fuel region due to fusion reactions and end losses is then given by:

$$
\begin{gathered}
\dot{\mathrm{N}}_{\mathrm{D}}=\dot{\mathrm{N}}_{D_{\text {Dffus }}}+\dot{\mathrm{N}}_{D_{\text {_ends }}} \\
\dot{\mathrm{N}}_{\mathrm{T}}=\dot{\mathrm{N}}_{\mathrm{T}_{-} \text {fus }}+\dot{\mathrm{N}}_{\mathrm{T}_{-} \text {ends }}
\end{gathered}
$$

The values of $\dot{\mathrm{N}}_{\mathrm{D}}$ and $\dot{\mathrm{N}}_{\mathrm{T}}$ are multiplied by the time step $\Delta \mathrm{t}$ and used to update the total ion number remaining at each time step.

The fusion power at each time step is then estimated by [30]:

$$
\mathrm{P}_{\mathrm{DT}}=\dot{\mathrm{N}}_{\mathrm{DT}} \cdot \mathrm{Q}_{\mathrm{DT}}
$$

which in turn is used to estimate the fusion energy released at each time step, $\mathrm{N}_{\mathrm{DT}}$. $\mathrm{Q}_{\mathrm{DT}}$. This energy value is then summed over the total simulation time to provide the total energy yield, which is used to provide estimates of engine performance (Section II.D).

\section{Analytic Model: Preliminary Results}

The simplified semi-analytic model was used to investigate the effects of various parameters on system performance, for example changing the initial target radius, density and velocity; changing the strengths of the magnetic field gradient and maximum external axial field; and evaluating the impacts of the various heating and cooling terms discussed in Section III.C. Given the approximations made in the simplified analytic model, the quantitative values discussed below are undoubtedly optimistic; however, the trends appear reasonable and can be used to provide guidance for more detailed numerical simulations to follow. ${ }^{7}$

\section{A. Model Check: Adiabatic Compression}

As this is a new concept there are no easily accessible codes or experiments against which to compare the analytic model results. A limited evaluation of model accuracy was performed by comparing the maximum predicted fuel temperature due to adiabatic compression against well-known analytic solutions for adiabatic compression heating. This provides some reassurance that key energy terms are being evaluated correctly, but again an appropriate amount of caution should be used with the quantitative analytic model values presented in this paper.

An exact analytic expression for heating an ideal gas by adiabatic compression alone is given by [31]:

$$
\mathrm{T}=\mathrm{T}_{0}\left(\frac{\mathrm{r}_{0}}{\mathrm{r}}\right)^{4 / 3}
$$

where $r_{0}$ and $r$ are the initial and compressed fuel radius, $T_{0}$ and $T$ are the initial and compressed fuel temperatures, respectively. Three test cases were run to compare the adiabatic compression temperatures predicted by the model against the exact analytic expression. The value of the compression ratio calculated by the model was used along with the initial fuel temperature to determine the final fuel temperature per Equation 52. In each case the target outer liner radius was set at $1-\mathrm{cm}$, liner thickness at $0.16-\mathrm{cm}$ (for an aspect ratio of 6), external vacuum field at 30-T, gradient field at $100-\mathrm{T} / \mathrm{m}$, and initial fuel density at $0.05-\mathrm{kg} / \mathrm{m}^{3}$. Initial fuel temperature and initial internal magnetic field values were changed to affect the maximum compression ratio. Results of the trials are presented in Table 2 below. In general the semi-analytic model does well at predicting the fuel temperatures due to adiabatic compression. While the remaining terms do not lend themselves as easily to an analytic comparison, the results for the adiabatic heating term provide some confidence in the solutions presented for the semi-analytic model.

\footnotetext{
${ }^{7}$ A significantly more capable 3D numerical simulation, SPFMax [32], is being developed by the University of Alabama Huntsville to model the physics of target-driven fusion systems, and will be used to investigate the concept in more detail.
} 
Table 2. Predicted and analytic adiabatic compression fuel temperatures for various initial conditions

\begin{tabular}{|c|c|c|c|c|c|}
\hline $\begin{array}{c}\text { Initial Fuel } \\
\text { Temperature } \\
(\mathrm{eV})\end{array}$ & $\begin{array}{c}\text { Initial Internal } \\
\text { Magnetic Field } \\
(\mathrm{T})\end{array}$ & $\begin{array}{c}\text { Fuel } \\
\text { Compression } \\
\text { Ratio (Model) }\end{array}$ & $\begin{array}{c}\mathrm{T}(\mathrm{eV}) \text { at } \\
\text { Compression } \\
\text { (Model) }\end{array}$ & $\begin{array}{c}\mathrm{T}(\mathrm{eV}) \text { at } \\
\text { Compression } \\
\text { (Eqn 48) }\end{array}$ & $\begin{array}{c}\% \\
\text { Difference }\end{array}$ \\
\hline 1 & 1 & 34.7 & 105 & 112.9 & 7.0 \\
\hline 1 & 2 & 17.2 & 42.8 & 44.3 & 3.4 \\
\hline 50 & 1 & 33.2 & $5.02 \times 10^{3}$ & $5.33 \times 10^{3}$ & 5.8 \\
\hline
\end{tabular}

\section{B. Initial Target Density}

Changing the initial target fuel density changes not only the amount of fuel present in the target, but also the compression dynamics. Low density targets may not provide sufficiently high reaction rates during compression for significant fusion to occur, limiting the yield; conversely, high internal pressures generated in higher density targets at lower fuel temperatures may prevent significant compression and fuel heating, again limiting reaction rates and energy yield.

The initial target density was stepwise evaluated from a low of $0.01 \mathrm{~kg} / \mathrm{m}^{3}$ to a high of $0.1 \mathrm{~kg} / \mathrm{m}^{3}$. The initial target injection velocity was 10-km/s, the outer (beryllium) liner radius was 1.0-cm with an aspect ratio of 6 (liner thickness of $0.16-\mathrm{cm}$ ), and a target length of $5-\mathrm{cm}$. The initial fuel temperature was set to $1-\mathrm{eV}$ with a maximum preheat temperature of $500-\mathrm{eV}$ (occurring as the target traversed the gradient region). The maximum axial magnetic field produced by the coil was set to $30-\mathrm{T}$, with an axial gradient of $100-\mathrm{T} / \mathrm{m}$ (e.g. rising from $1-\mathrm{T}$ to $30-\mathrm{T}$ in approximately $30-\mathrm{cm}$ ), and the initial internal target axial magnetic field was set to $1-\mathrm{T}$. The only change in initial conditions for each run was the fuel density, and the impact on performance was primarily measured by the impact on yield, impulse, and specific impulse for each pulse. The change in specific impulse (Isp) with initial target density is plotted in Fig. 7 below.

For the same initial conditions, the specific impulse increases with increasing fuel density up to approximately $0.07-\mathrm{kg} / \mathrm{m}^{3}$, at which point the Isp rapidly decreases. Reviewing the runs at higher density values, the compressed fuel temperatures were limited to a few $\mathrm{keV}$, too low for any significant fusion events to occur. As such the target essentially underwent some radial compression, but with no significant additional heating as it traversed the coil and exited the system. An initial target fuel density of around $0.07 \mathrm{~kg} / \mathrm{m}^{3}(0.07 \mathrm{mg} / \mathrm{cc})$ thus appears close to optimum for the system under evaluation, and was used for the subsequent performance trades discussed below.

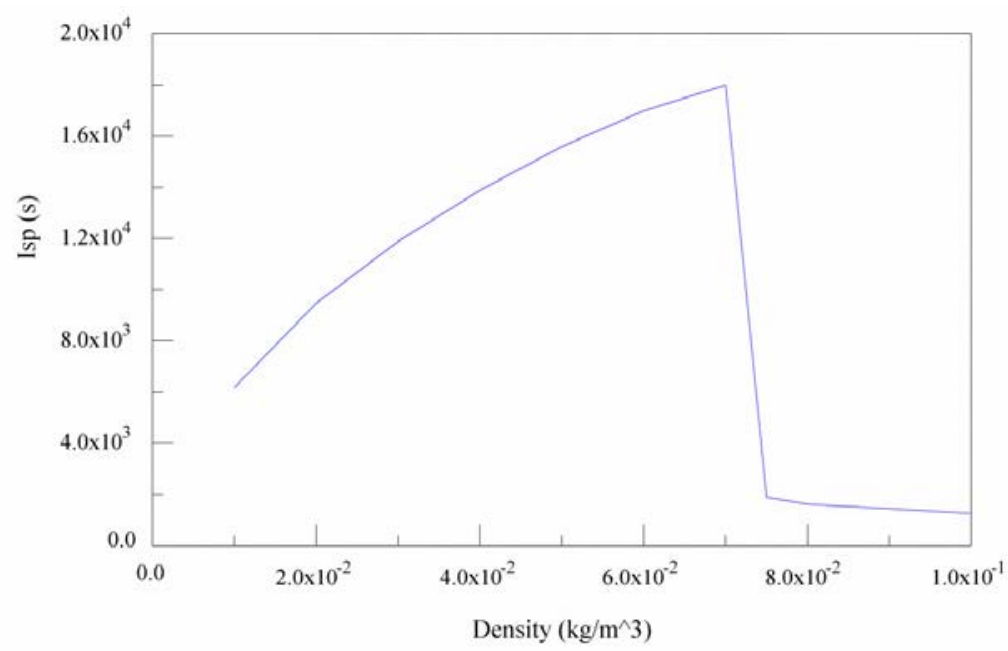

Fig. 7 Specific Impulse as a function of initial fuel density

\section{Initial Target Radius}

Keeping the density constant at $0.07-\mathrm{kg} / \mathrm{m}^{3}$ and the other values as described above, the external target radius was sequentially changed from $0.75-\mathrm{cm}$ to $2.0-\mathrm{cm}$. The aspect ratio (ratio of external target radius to liner thickness) was kept at $\mathrm{AR}=6$ for each run, corresponding to the minimum AR value for stability quoted in the literature. The impact of changing radius on performance parameters was then evaluated, and the change in specific impulse as a function of target radius is shown in Fig. 8. 


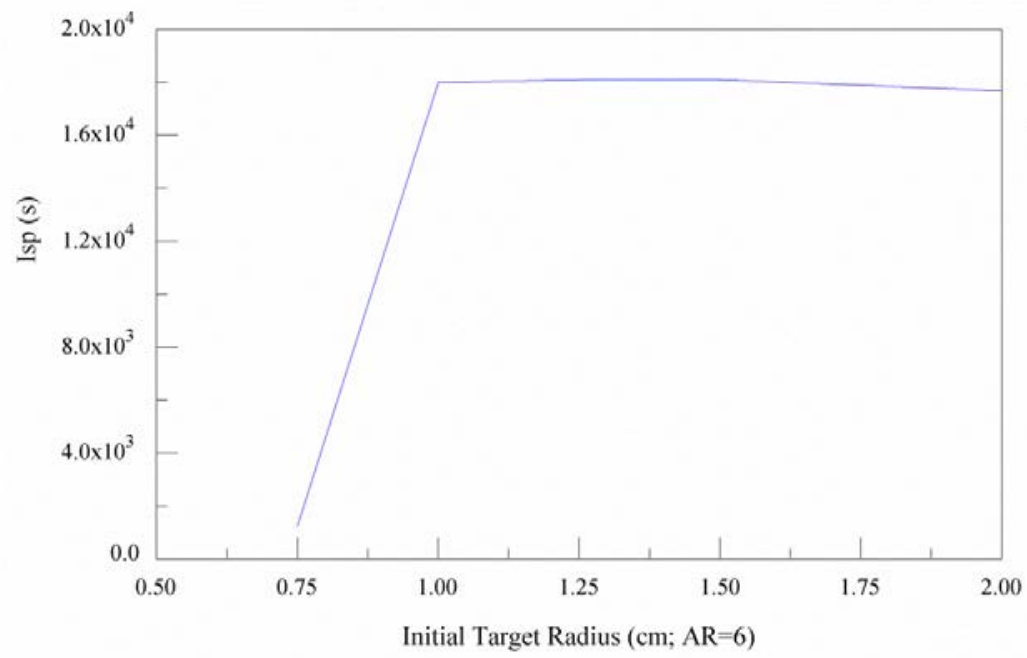

Fig. 8 Specific impulse as a function of initial target radius.

As shown in the figure, the Isp rapidly increases over a small change in initial radius, changing from approximately $1,000 \mathrm{~s}$ at $0.75 \mathrm{~cm}$ to a value approaching $18,000 \mathrm{~s}$ at a target radius of $1 \mathrm{~cm}$, after which there is no significant change in performance with increasing target radius. As noted in Appendix B, the yield at an initial target radius of $0.75 \mathrm{~cm}$ is approximately 4 orders of magnitude lower than the yield for target radii larger than $1 \mathrm{~cm}$; for the assumed parameters, the small radius target was unable to reach the required compression densities and temperatures for significant fusion events to occur.

\section{Initial Target Velocity}

As described in Section I, the rapidly time changing magnetic field used in standard $\theta$-pinch experiments to cause compression is replaced in this system with a conductively lined fuel target injected at high velocity along the axis of a superconducting coil; the combination of axial velocity and axial magnetic field gradient provide an effective time changing magnetic field in the target frame of reference. As discussed in Section II.C, the time it takes the axial magnetic field to diffuse through the conductive liner is longer than the target compression time, and the unbalanced external pressure causes the target to implode. To evaluate the impact of initial target velocity on system performance, the target velocity was evaluated from $2 \mathrm{~km} / \mathrm{s}$ to $20 \mathrm{~km} / \mathrm{s}$, assuming a constant magnetic field gradient of $100-\mathrm{T} / \mathrm{m}$. The initial target density was $0.07-\mathrm{kg} / \mathrm{m}^{3}$ and the radius was set to $1-\mathrm{cm}$ for each run, with all other values the same as above. The results of changing the initial target velocity on specific impulse are shown in Fig. 9 below.

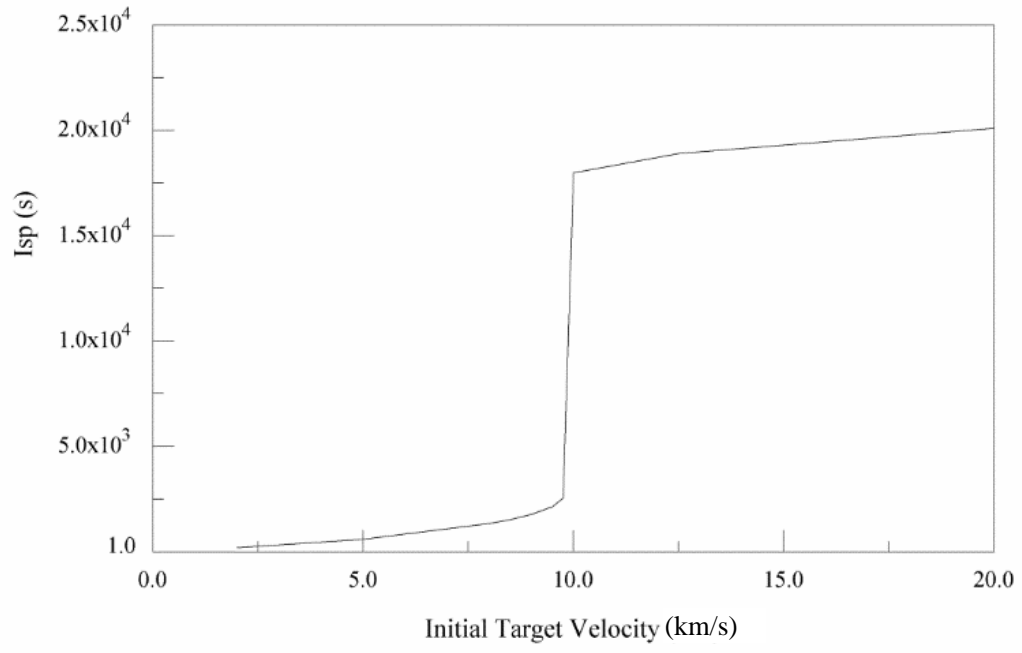

Fig. 9 Specific impulse as a function of initial target velocity 
For low target velocities the Isp remains approximately equal to that of the initially accelerated target traversing the systems without additional energy gain. However, at approximately $10-\mathrm{km} / \mathrm{s}$ there is significant additional energy gain from fusion events, with the Isp rapidly increasing to approximately 18,000 s, and then slowly increasing at higher initial velocities. At the lower injection velocities, the apparent change in magnetic field does not increase sufficiently rapidly for significant compression to occur. At around $10-\mathrm{km} / \mathrm{s}$, the changing magnetic field in the target frame of reference is sufficient to compress the target and initiate fusion, providing rapid additional energy gain and a correspondingly large increase in specific impulse. Beyond 10-km/s there is minimal additional gain, accounting for the slower increase in Isp with increasing initial velocity. To minimize the amount of laser power required to accelerate the target, it appears that providing an initial target velocity of $10-\mathrm{km} / \mathrm{s}$ may be sufficient to enable Isp values of interest.

\section{E. Target Preheating}

Prior studies have shown the benefit of preheating the target fuel prior to compression, to reduce the amount of compression required to reach fusion temperatures. To evaluate the effect of initial fuel temperature on performance, it was assumed that once the target reaches its injection velocity, a second high energy laser pulse would be focused on the aft end of the target to generate a specified amount of heating as the target traversed the gradient field region (Section III.C). To evaluate the effect of fuel preheating on performance, the amount of preheating was varied from $50-\mathrm{eV}$ to $750-\mathrm{eV}$, assuming a uniform temperature distribution within the fuel region. The initial target density was $0.07-\mathrm{kg} / \mathrm{m}^{3}$, with an outer radius of $1-\mathrm{cm}$ and initial injection velocity of $10-\mathrm{km} / \mathrm{s}$. The remaining values are the same as above. The impact on specific impulse by laser preheating of the fuel is shown in Fig. 10 below.

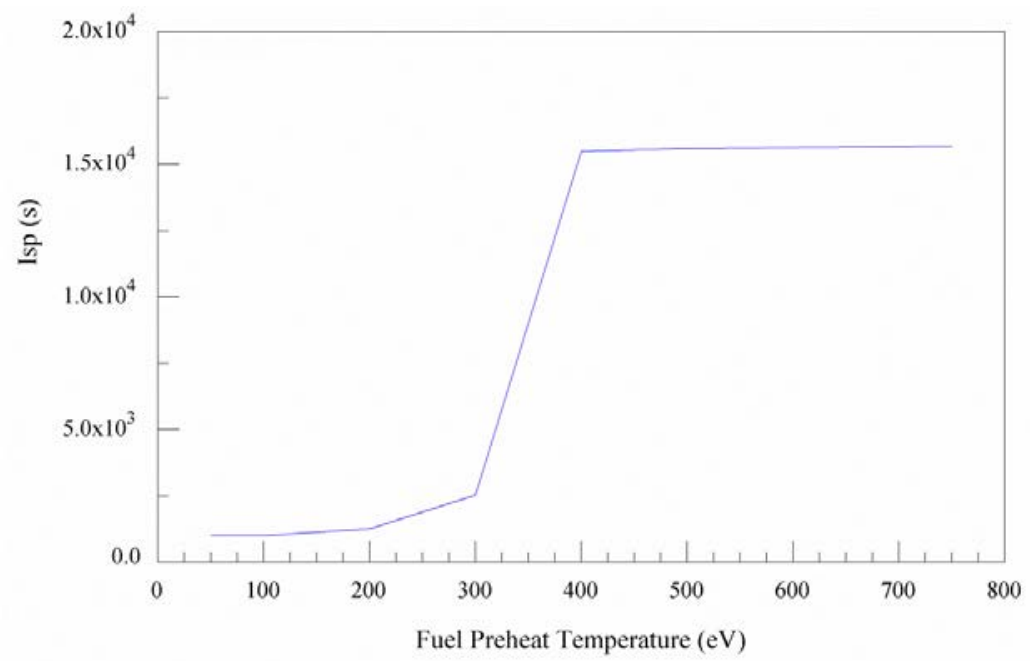

Fig. 10 Specific impulse as a function of uniformly preheated fuel temperature

For initial fuel temperatures below approximately $300 \mathrm{eV}$, very little additional energy gain is provided by the system; the compression of the target is insufficient to raise the initial temperature high enough for significant amounts of fusion to occur. Between $300 \mathrm{eV}$ and $400 \mathrm{eV}$, the Isp rapidly increases as the compressed fuel conditions begin to support gainful fusion. Above roughly $400 \mathrm{eV}$ there is no significant increase in Isp with increasing initial fuel temperature for otherwise identical targets. Higher initial fuel temperatures would require a commensurate increase in laser heating power, with little additional benefit to system performance. Hence preheating the target to approximately $400 \mathrm{eV}$ appears sufficient to generate a significant fusion yield on compression.

\section{F. External Magnetic Field}

The magnetic field produced by the superconducting solenoid coil creates the external axial magnetic field required to maintain inward radial pressure on the target during compression and heating. To evaluate the effect of external magnetic field strength on performance, the value of the maximum axial magnetic field was varied from 5$\mathrm{T}$ to $50-\mathrm{T}$. For these runs, the initial fuel density, radius, and target velocity were $0.07-\mathrm{kg} / \mathrm{m}^{3}, 1-\mathrm{cm}$, and $10-\mathrm{km} / \mathrm{s}$, respectively, with a fuel preheat temperature of $500-\mathrm{eV}$. A magnetic field gradient of $100-\mathrm{T} / \mathrm{m}$ was assumed in all cases. The results of changing the maximum external magnetic field on specific impulse are plotted in Fig. 11. 
Below approximately 30-T, there is little increase in specific impulse beyond that of the uncompressed target exiting the system. At approximately 30-T, sufficient radial compression is achieved to initiate substantial fusion reactions, resulting in a significant increase in energy yield and corresponding increase in directed exhaust velocity, again assuming a 70\% magnetic nozzle conversion efficiency. Increasing the external magnetic field beyond 30-T does not significantly increase the specific impulse, and would require more advanced steady-state superconducting magnets than currently available. While higher external compression fields can be achieved using pulsed coils, the intent of the current study was to assess the viability of a steady state superconducting magnet to alleviate the mass and complexities associated with high current pulsed power delivery systems and resistive coils. It appears that an axial magnetic field of approximately 30-T, currently at the limit of available superconducting magnet technology [27], may be sufficient for the concept to work.

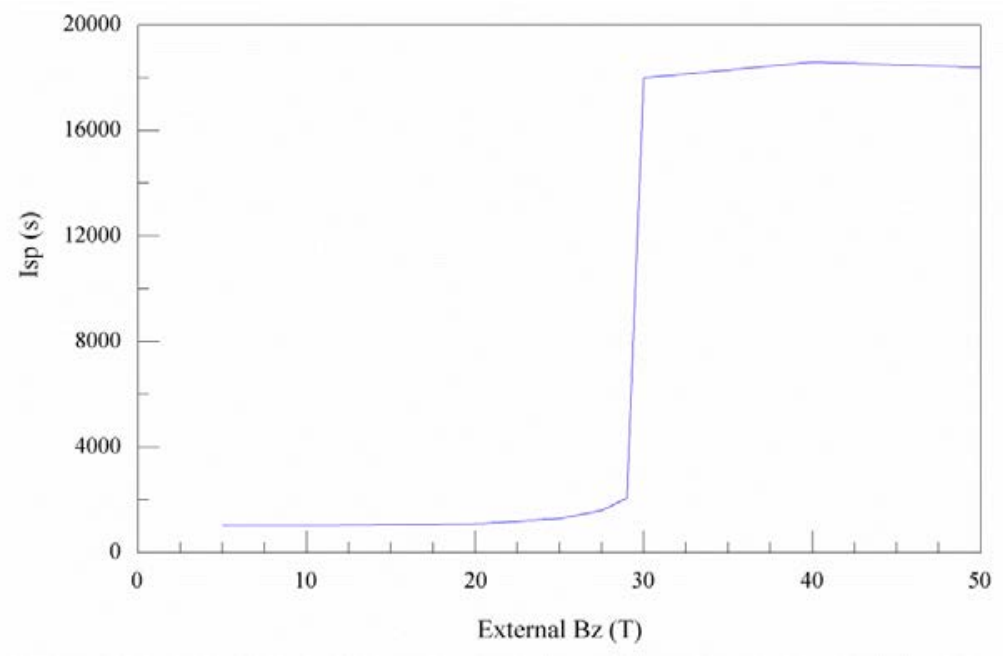

Fig. 11 Specific impulse as a function of axial magnetic field external to the target

\section{G. Internal Target Magnetic Field}

As noted in the prior discussion of pressure and energy terms (Section III), an initial axial magnetic field within the target prior to compression will increase as the square of the compression ratio (Equation 20), assuming no magnetic field diffusion through the target occurs during the compression time. To evaluate the effect of initial internal magnetic field strengths on systems performance, the internal magnetic field was varied from 1-T to 5-T, and the impact on specific impulse plotted in Fig. 12.

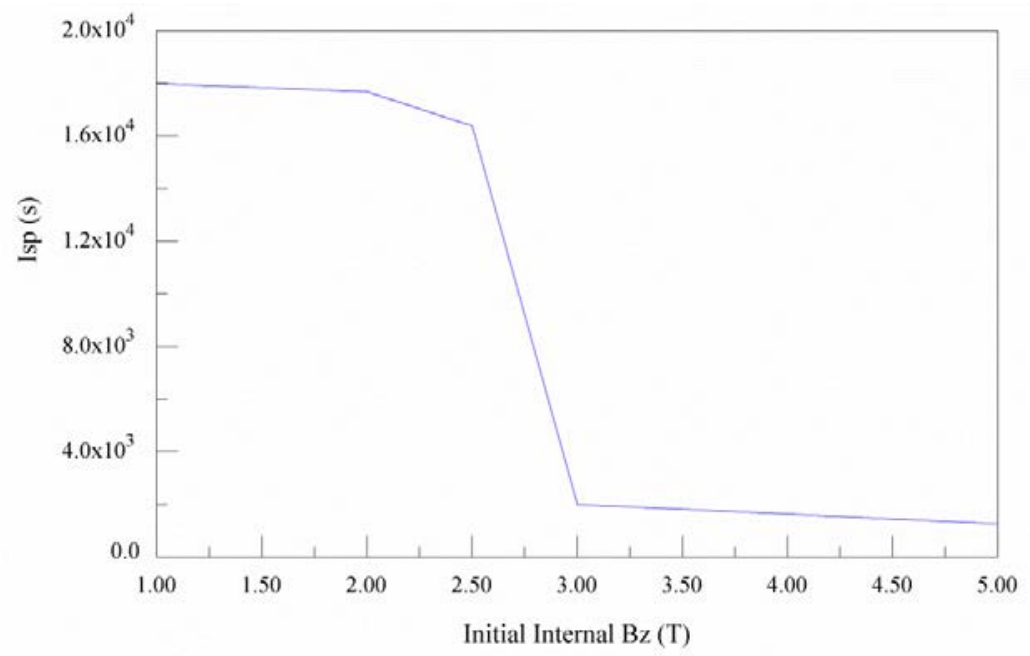

Fig. 12 Specific impulse as a function of initial target internal magnetic field 
It is seen that the specific impulse drops off fairly rapidly for initial internal target magnetic fields above approximately 2.5-T. An inquiry of the code results shows that above this value, and for the target and external magnetic field parameters described above, the maximum radial fuel compression is lower for higher initial target internal magnetic fields. As a specific comparison, the simple analytic model predicts a maximum fuel compression ratio of around 14 with an initial internal field of 2.5-T, while the compression ratio is closer to 6 with an initial internal field of 5-T. The lower amount of compression corresponds to a lower maximum fuel density and temperature, resulting in less fusion reactions, lower yield, and lower specific impulse. An internal magnetic field of approximately 1-T appears to provide a suitably high field on compression to mitigate radial thermal conduction and provide trapped $\alpha$-particle heating.

\section{H. Magnetic Field Gradient}

As discussed in Section I, the concept uses a stationary magnetic field and rapidly injected target to generate the compression dynamics of more standard $\theta$-pinch geometries. The axial target velocity and magnetic field gradient combine to mimic a rapidly time-changing magnetic field in the moving target frame of reference, creating the conditions necessary for radial compression. Assuming a $10-\mathrm{km} / \mathrm{s}$ initial target velocity, 30-T external axial magnetic field, 1-T initial internal magnetic field, and a $1-\mathrm{cm}$ radius, $0.07-\mathrm{kg} / \mathrm{m}^{3}$ target preheated to $500-\mathrm{eV}$ (used for consistency during the trade study), the impact of magnetic field gradient on specific impulse is plotted in Fig. 13 below.

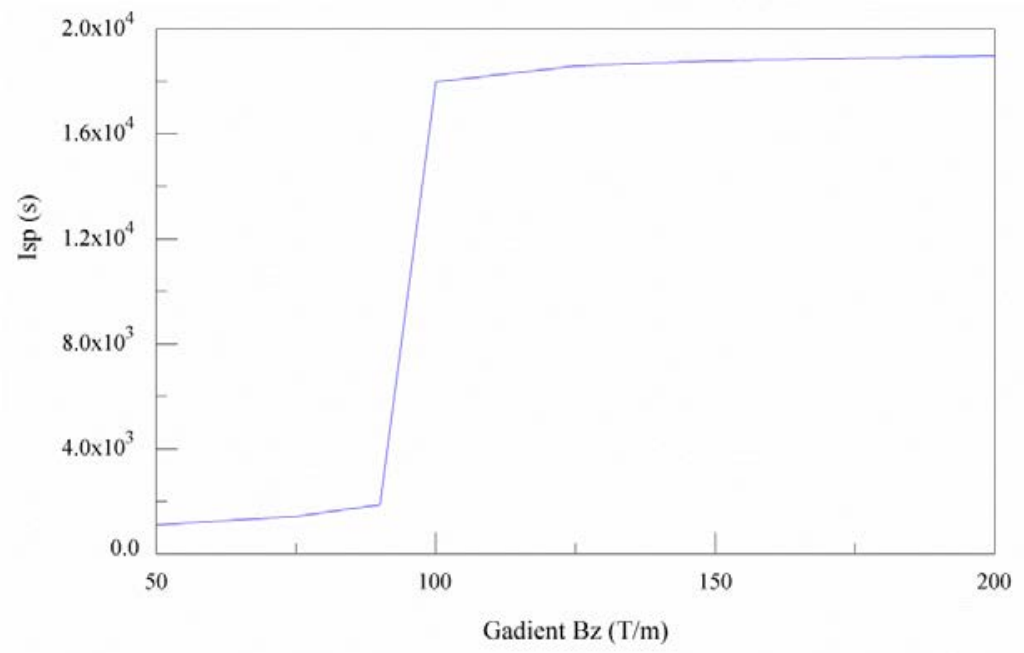

Fig. 13 Specific impulse as a function of axial magnetic field gradient

The axial gradient of the magnetic field provided by the superconducting coil was varied from $50-\mathrm{T} / \mathrm{m}$ to 200 $\mathrm{T} / \mathrm{m}$, with a sharp increase in specific impulse observed for a gradient field value of around $100-\mathrm{T} / \mathrm{m}$. At this value, the combination of initial target velocity and magnetic field gradient generated sufficient target compression for fusion reactions to occur, resulting in significant energy gain and a rapid increase in specific impulse. Above 100$\mathrm{T} / \mathrm{m}$ the energy produced by the target (assuming the same initial density, radius, etc.) doesn't significantly increase, but an inquiry of the code results shows the time to reach maximum compression was shorter (e.g. approximately $42-\mu \mathrm{s}$ at $100-\mathrm{T} / \mathrm{m}$ vs. $32-\mu \mathrm{s}$ at $200-\mathrm{T} / \mathrm{m}$ ). The combination of target velocity and higher field gradient appears equivalent to a higher rate of change of magnetic field in the target frame of reference, resulting in more rapid radial compression.

\section{Liner Material}

Finally, the simplified model was run with 3 different types of liner material: aluminum (Al), beryllium (Be), and lithium ( $\mathrm{Li}$ ), to evaluate the effect of liner mass on target compression and corresponding system performance. The runs described in the sections above were performed with a Be liner, which is a good neutron reflector; the literature also reports models and experiments using $\mathrm{Li}$ and $\mathrm{Al}$ liners, so these were also evaluated in a limited set of runs for comparison with Be. No attempt was made to evaluate the various radiative or reflective properties of each liner; the comparisons were strictly to observe the effects of different liner densities on target compression. The set of generally optimized values found in the preceding sections, summarized here, were used in each liner simulation: 
Table 3. Target and magnetic field values used in liner material evaluation

\begin{tabular}{|c|c|c|c|c|c|c|c|}
\hline $\begin{array}{c}\text { Initial } \\
\text { Fuel } \\
\text { Density }\end{array}$ & $\begin{array}{c}\text { Initial } \\
\text { Liner } \\
\text { Radius }\end{array}$ & $\begin{array}{c}\text { Aspect } \\
\text { Ratio }\end{array}$ & $\begin{array}{c}\text { Injection } \\
\text { Velocity }\end{array}$ & $\begin{array}{c}\text { Fuel } \\
\text { Preheat } \\
\text { Temp }\end{array}$ & $\begin{array}{c}\text { External B- } \\
\text { Field }\end{array}$ & $\begin{array}{c}\text { Initial } \\
\text { Internal B- } \\
\text { Field }\end{array}$ & $\begin{array}{c}\text { Axial B- } \\
\text { Field } \\
\text { Gradient }\end{array}$ \\
\hline $0.07 \mathrm{~kg} / \mathrm{m}^{3}$ & $1.0 \mathrm{~cm}$ & 6 & $10 \mathrm{~km} / \mathrm{s}$ & $500 \mathrm{eV}$ & $30 \mathrm{~T}$ & $1.0 \mathrm{~T}$ & $100 \mathrm{~T} / \mathrm{m}$ \\
\hline
\end{tabular}

To evaluate the effect of liner material on system performance, plots were obtained not only for specific impulse but also for the yield and impulse provided per pulse.

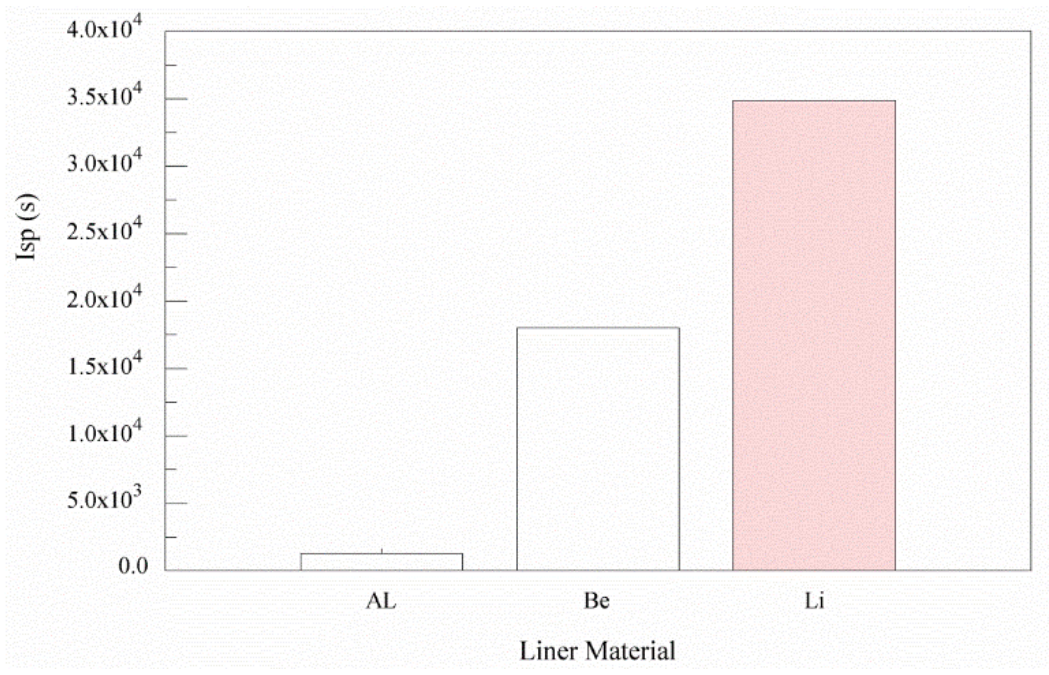

Fig. 14 Specific impulse as a function of target liner material

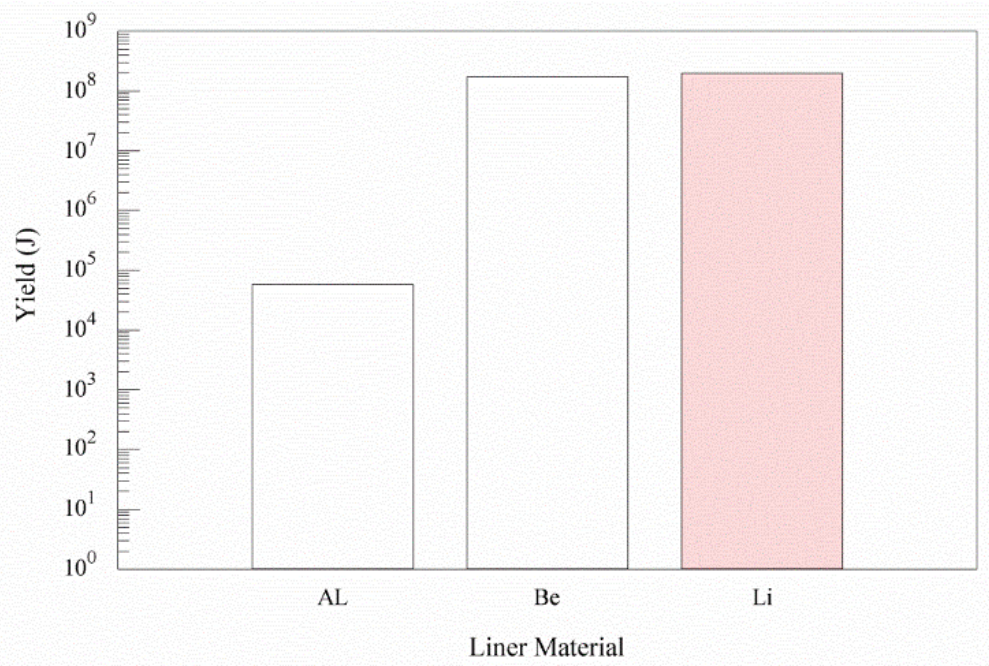

Fig. 15 Yield as a function of target liner material 


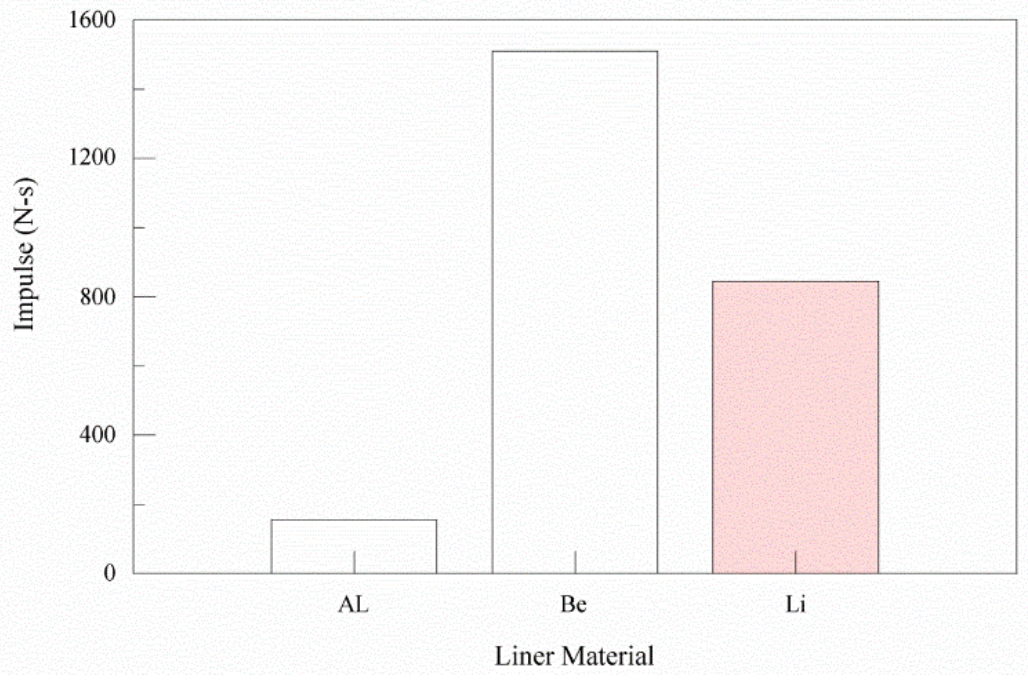

Fig. 16 Impulse as a function of target liner material

Of interest, the aluminum liner didn't achieve a significant energy yield under the assumed target and magnetic field conditions, while both the Be and Li lined targets reached similarly high energy yields $\left(1.7 \times 10^{8} \mathrm{~J}\right.$ and $1.95 \times 10^{8}$ $\mathrm{J}$, respectively). Because the $\mathrm{Al}$ liner didn't achieve any appreciable energy gain, the specific impulse was similar to that of the initially accelerated target traversing the system. In the remaining two cases the specific impulse increases as the liner material density decreases; e.g. the Be lined target delivered approximately 18,000 s, compared to roughly 34,900 s for the Li lined target. The target properties outlined above correspond to a Be liner mass of $8.60 \times 10^{-3} \mathrm{~kg}$ and Li liner mass of $2.47 \times 10^{-3} \mathrm{~kg}$; the DT fuel mass in each case is approximately $7.76 \times 10^{-7} \mathrm{~kg}$, hence the target mass is dominated by the liner mass. Because the liner mass makes up the bulk of the total target mass, higher mass targets will have a lower directed exhaust velocity than lower mass targets for a given yield energy. The impulse delivered for each case shows the Li lined target has a lower impulse than the Be lined target (845 N-s vs. $1510 \mathrm{~N}$-s, respectively), which again is primarily due to the lower mass of the Li liner. Per Equation 12, the impulse is directly proportional to the exhaust mass and specific impulse; substituting the values of mass and specific impulse for each target into that equation provides the impulse values noted in Fig. 16. Based on the analytic model predictions, a lithium liner provides roughly a factor of 2 increase in specific impulse but about half the impulse of a beryllium liner for an otherwise similar D-T target.

\section{J. Pulse Considerations}

The results presented above were obtained assuming a total run time of $100-\mu$ s for each case, however the target may undergo compression followed by rapid expansion and a subsequent second compression during longer simulation periods, as shown in Fig. 17. The latter compression cycle in the model results from the target cooling on expansion to the point where external magnetic field pressure on the axially moving target can again cause contraction. This is most likely an artifact of the simplified analytic model, but even were it to physically occur it would be more desirable to have the initial rapid expansion of the high temperature target (at this point a hot, radiating plasma) occur in the magnetic nozzle to provide directed thrust, rather than a rapid expansion within the confined bore of the superconducting coil. 


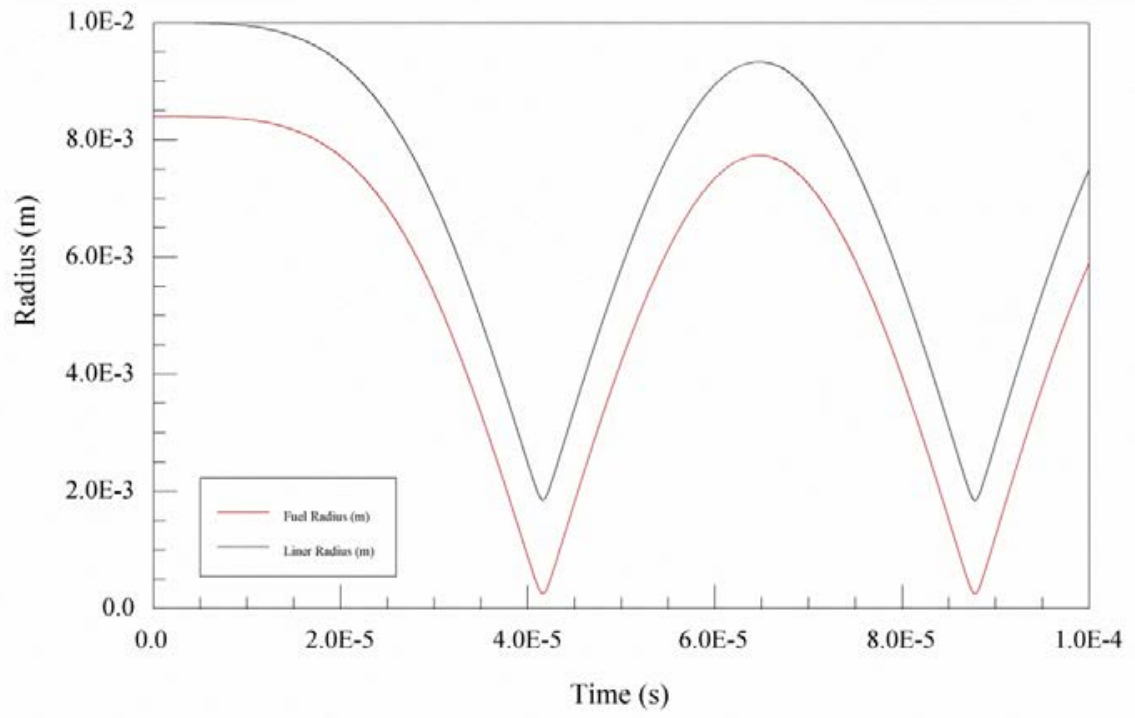

Fig. 17 Target radius compression, expansion, and subsequent compression cycle

As shown in Fig. 17, the first compression cycle reaches a minimum radius at a time of approximately $41.5-\mu$ s (the time between the target entering the magnetic field gradient and the time for maximum compression to occur). At this point the target begins to expand, and if located in the magnetic nozzle the radially expanding plasma would be redirected to provide axial thrust. This indicates the superconducting coil preceding the magnetic nozzle should be relatively short; for an initial target velocity of $10-\mathrm{km} / \mathrm{s}$, the magnet coil will be just over $0.4 \mathrm{~m}$ long. While this estimate needs to be verified with more detailed numerical modeling, it does indicate that reasonable superconducting magnet sizes and field strengths may be employed for the concept.

Although the initial compression time for the target is on the order of several tens of microseconds, the period over which the target is accelerated to its axial injection velocity can be significantly longer, subject to the engine's pulse repetition frequency. Returning to Section II.B, a rough estimate can be made of the time required to accelerate the target to the desired injection velocity. The ablative acceleration model discussed in that section also derives the acceleration time $(\mathrm{t})$ as a fraction of the time required to fully burn through the ablative target material $\left(\mathrm{t}_{\mathrm{b}}\right)$, which is related to the fraction of remaining target mass $\left(\mathrm{m}_{\mathrm{f}}\right)$ to initial target mass $\left(\mathrm{m}_{0}\right)$ :

$$
\left(1-\mathrm{t} / \mathrm{t}_{\mathrm{b}}\right)=\mathrm{m}_{\mathrm{f}} / \mathrm{m}_{0}
$$

The burn time is given by:

$$
\mathrm{t}_{\mathrm{b}}=\left(\frac{\mathrm{L}_{0}}{\mathrm{C}_{\mathrm{c}}}\right)\left(\frac{\mathrm{n}_{0}}{\mathrm{n}_{\mathrm{c}}}\right)
$$

where Lo is the target diameter, Cc is the sound speed of the target material at the critical density $\mathrm{n}_{\mathrm{c}}$, and $\mathrm{n}_{0}$ is the initial density of the ablated material. The critical sound speed is given by:

$$
\mathrm{C}_{\mathrm{c}}=\left(\frac{\mathrm{I}}{2 \mathrm{n}_{\mathrm{c}} \mathrm{m}_{\mathrm{i}}}\right)^{1 / 3}
$$

where $\mathrm{I}$ is the incident laser intensity $\left(\mathrm{W} / \mathrm{m}^{2}\right)$, and $\mathrm{m}_{\mathrm{i}}$ is the mass of an ablated ion (in this case, Be). From Section II.B, the critical density for a $\mathrm{CO}_{2}$ laser is $\mathrm{n}_{\mathrm{c}}=2.52 \times 10^{23} \mathrm{~m}^{-3}$. The density of $\mathrm{Be}$ is $1850 \mathrm{~kg} / \mathrm{m}^{3}$, and the ion mass is $1.5 \times 10^{-26} \mathrm{~kg}$, for an initial number density of $1.23 \times 10^{29} \mathrm{~m}^{-3}$. Following Section II.B a laser power of $10-\mathrm{kW}$ provides a laser intensity on a $1-\mathrm{cm}$ radius target of $3.14 \times 10^{7} \mathrm{~W} / \mathrm{m}^{2}$. Equation 7 then predicts a velocity of $1.8 \times 10^{5} \mathrm{~m} / \mathrm{s}$ for the ablated Be ions. Substituting this into Equation 8 indicates approximately $9 \%$ of the Be liner material is ablated to reach the required injection velocity of $10 \mathrm{~km} / \mathrm{s}$. Using Equation 55, the critical sound speed is equal to $1.1 \times 10^{3} \mathrm{~m} / \mathrm{s}$, and assuming a $10^{-2} \mathrm{~m}$ radius target, Equation 54 then yields a burn time of $8.8 \mathrm{~s}$. From Equation 53 , the time required to accelerate the target, with $\mathrm{m}_{\mathrm{f}} / \mathrm{m}_{0}=0.91$, is $0.76 \mathrm{~s}$, substantially longer than the desired $0.1 \mathrm{~s}$ acceleration 
period. This indicates the laser power must be increased to more rapidly accelerate the target. Redoing the above calculations and assuming a laser power of 1-MW results in a laser intensity on target of $3.2 \times 10^{9} \mathrm{~W} / \mathrm{m}^{2}$. The corresponding critical sound speed is $7.5 \times 10^{3} \mathrm{~m} / \mathrm{s}$, and the ablated material velocity is $1.84 \times 10^{6} \mathrm{~m} / \mathrm{s}$. The ratio of final to initial mass from Equation 6 is 0.991 , with about $1 \%$ of the target mass ablated to reach the desired $10 \mathrm{~km} / \mathrm{s}$ injection velocity with the 1-MW laser. Using Equation 54 the burn time is approximately $1.3 \mathrm{~s}$, and from Equation 53 the acceleration time is predicted to be $1.17 \times 10^{-2} \mathrm{~s}$, or roughly $12 \mathrm{~ms}$. Similar calculations using lithium as the ablative material show that a similar amount of ablative mass $(\sim 1 \%)$ is required to reach an injection velocity of 10 $\mathrm{km} / \mathrm{s}$ with the 1-MW laser, but over a shorter acceleration period of $3.6 \mathrm{~ms}$. Although very crude estimates, the analysis indicates that a sufficiently high power laser incident on a Be or Li lined target for several milliseconds will rapidly ablate a small fraction of the liner material, providing sufficient axial acceleration of the target into the gradient field on a time scale suitable for multi-Hz pulsed operation.

\section{K. Optimized Case}

Based on the trades described above, an initial set of "optimum" target and magnetic field values were defined to help guide future, more detailed simulations of the D-T target implosion physics and associated vehicle and mission designs. These values were used in additional runs of the analytic model with an abbreviated simulation time of 45$\mu \mathrm{s}$, to capture target compression and the subsequent start of target dissociation/expansion. Separate runs were conducted for lithium and beryllium lined D-T targets with otherwise identical target and magnetic field values to estimate the potential range of engine performance. Figures 18-24 provide comparisons of several key parameters for the lithium and beryllium lined targets.

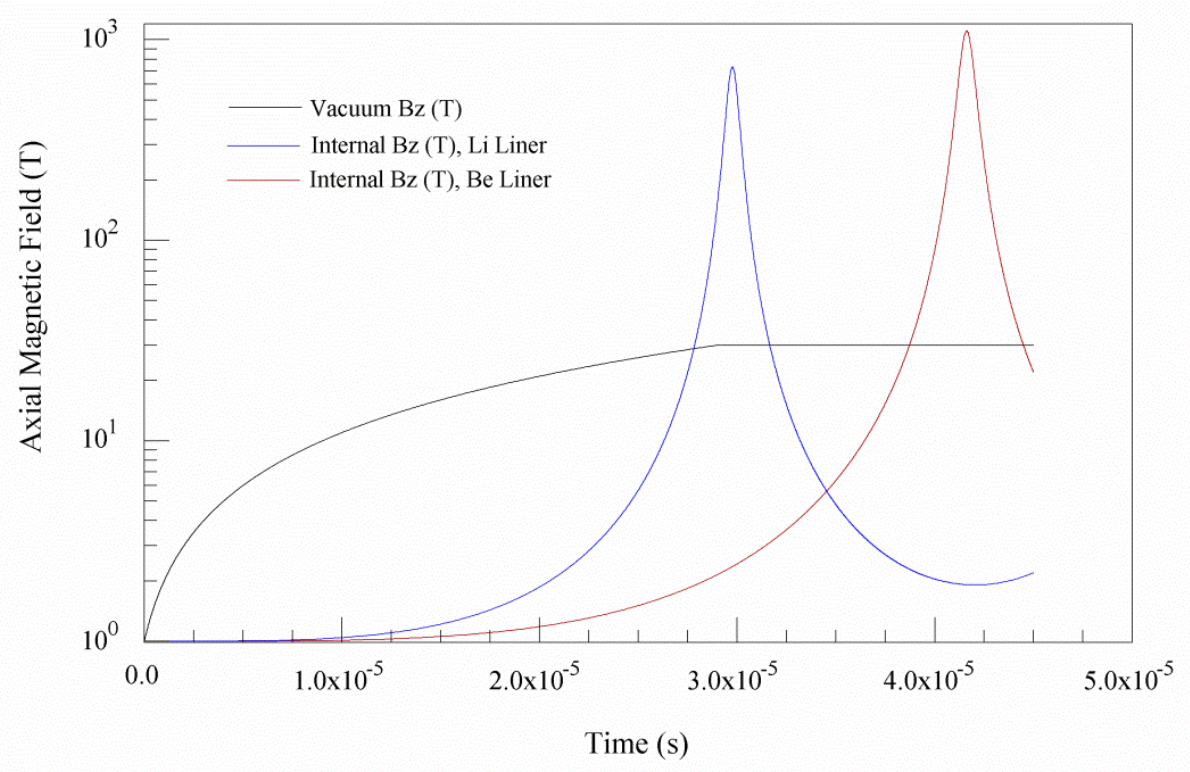

Fig. 18 External and internal magnetic field values for Be and Li lined targets

As shown in Fig. 18, the external magnetic field applied by the superconducting coil is assumed constant for each case, with an axial gradient of $100-\mathrm{T} / \mathrm{m}$ leading to a maximum value of $30-\mathrm{T}$ within the coil. During compression the initial 1-T axial field within the lithium lined target briefly reaches a maximum compressed value of approximately $730 \mathrm{~T}$, while the internal axial field within the beryllium lined target reaches a maximum value of around $1100 \mathrm{~T}$ due to a slightly higher amount of fuel compression, as seen in Fig. 19 below.

Of interest, the lithium lined target reaches maximum compression at an earlier time that the beryllium lined target. This is due to a more rapid radial acceleration of the lower mass lithium liner for a given compression force, compared to the beryllium lined target. 


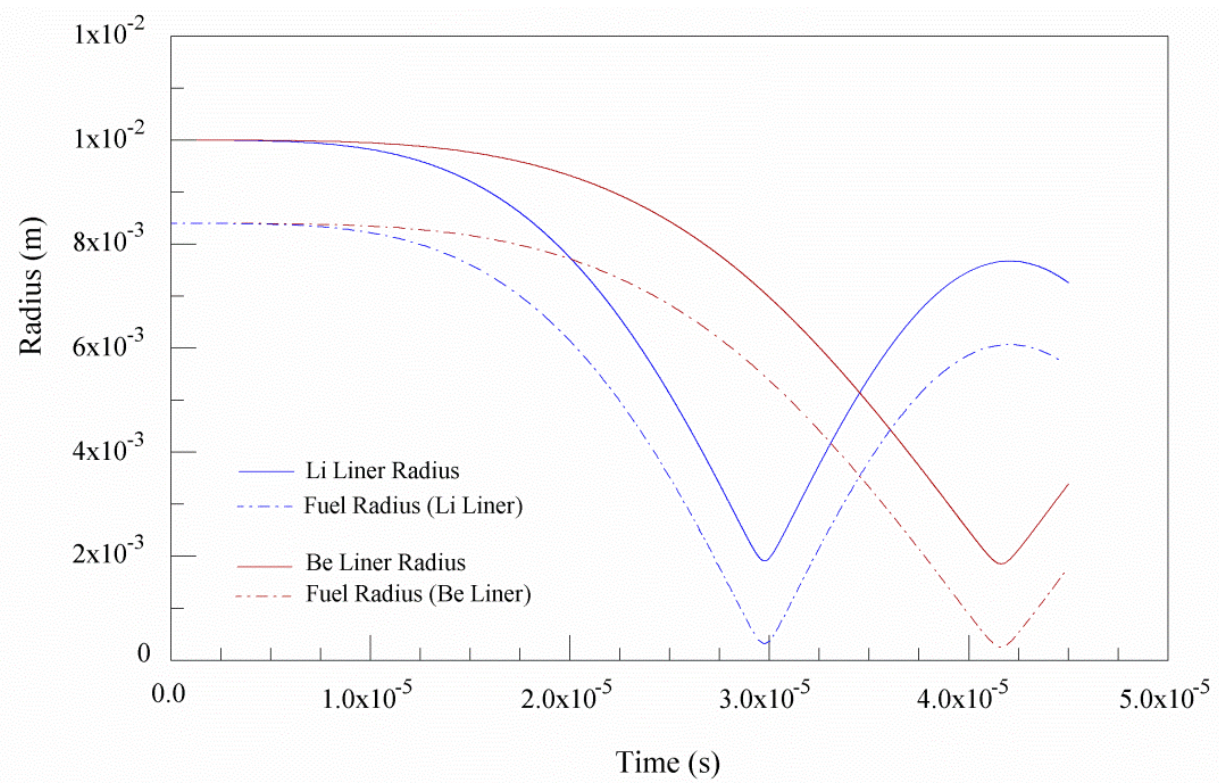

Fig. 19 Evolution of liner and fuel radius with time

Figure 20 displays the evolution of the D-T fuel density during compression; for the lithium lined target the fuel density increases from the initial $0.07 \mathrm{~kg} / \mathrm{m}^{3}$ to a value of approximately $37 \mathrm{~kg} / \mathrm{m}^{3}$, while for the beryllium lined target the fuel density increases to a value of $57 \mathrm{~kg} / \mathrm{m}^{3}$ before the target begins to expand.

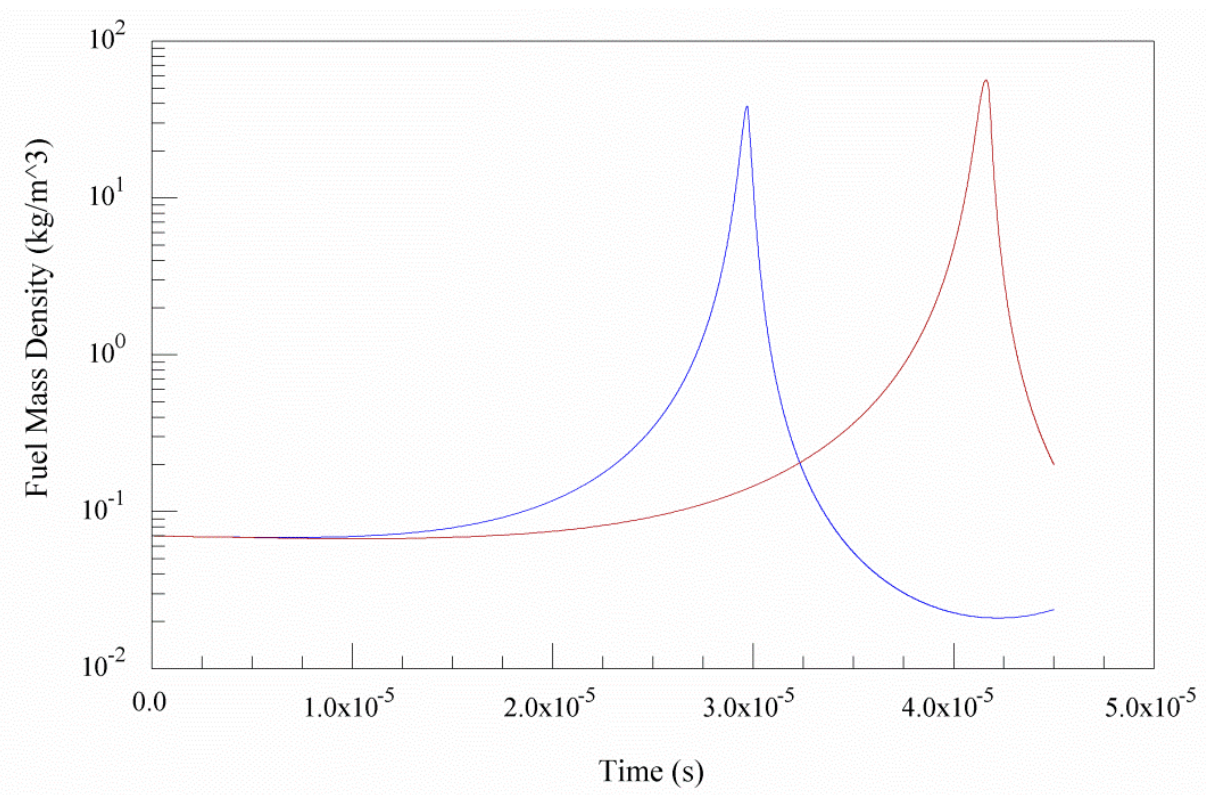

Fig. 20 Evolution of D-T fuel mass density with time

Figure 21 displays the evolution of the D-T ions due to fusion and end losses during compression (Section III.E). For a 50:50 mixture of D-T, the evolution of ion number is identical for each species. 


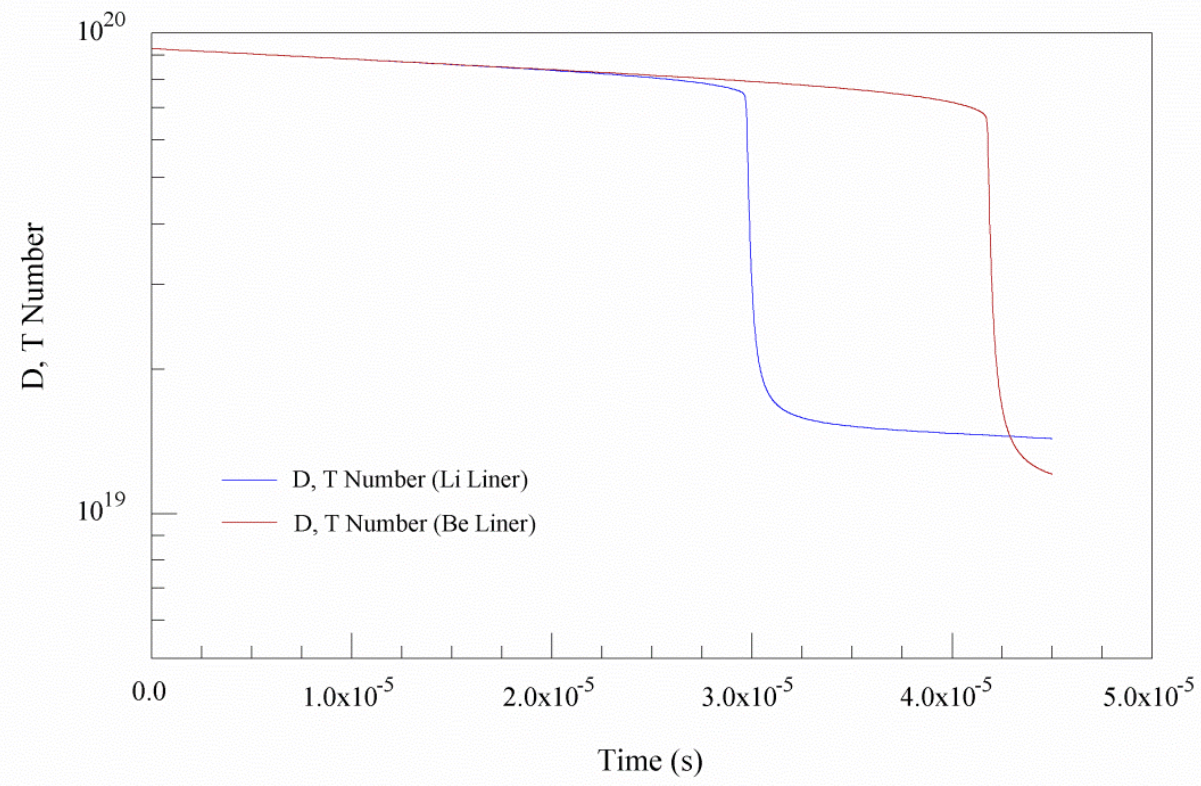

Fig. 21 Evolution of number of $D$, $T$ ions with time

Figure 22 shows the evolution of the D-T fuel temperature as the target is compressed, with an expanded vertical scale to more clearly show the evolution. In each case there is an initial temperature increase from $1 \mathrm{eV}$ to $500 \mathrm{eV}$ due to laser preheating, followed by heating due to adiabatic compression and a rapid increase in temperature as fusion begins to occur and the energy of the resulting $\alpha$-particles is trapped within the target and contributes to the rising fuel temperature. As the temperature rapidly increases the internal fuel pressure eventually exceeds the compressive magnetic field force to cause the target to expand, reducing the internal fuel temperature.

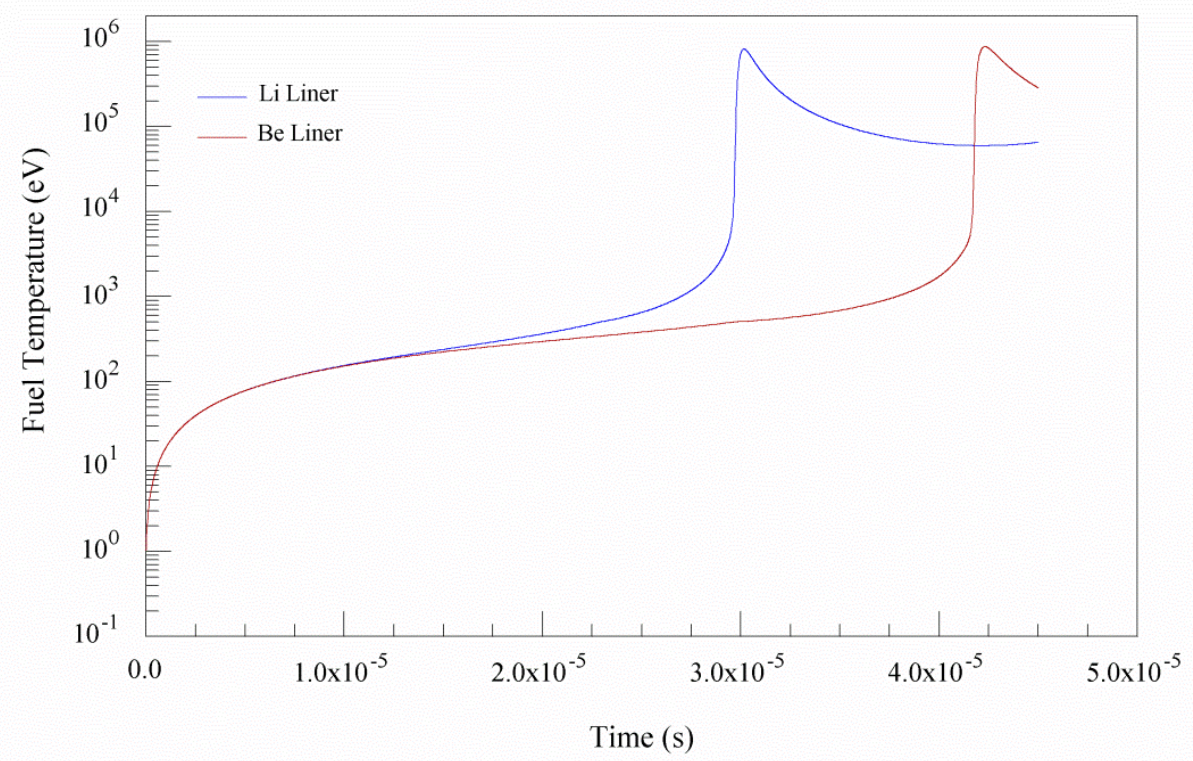

Fig. 22 Evolution of D-T fuel temperature with time

Figure 23 displays the areal density of the fuel during compression and subsequent expansion, where the fuel areal density is equal to the fuel mass density multiplied by the fuel radius $(\rho R)$. Upon compression the lithium lined target reaches a maximum $\rho \mathrm{R}$ value of $0.12 \mathrm{~g} / \mathrm{cm}^{2}\left(1.2 \mathrm{~kg} / \mathrm{m}^{2}\right)$, while the beryllium lined target reaches an areal density of approximately $0.14 \mathrm{~g} / \mathrm{cm}^{2}\left(1.4 \mathrm{~kg} / \mathrm{m}^{2}\right)$. These values (and corresponding fuel temperatures) are comparable to the $\rho \mathrm{R}$ values for net energy production shown in the Lindl-Widner plot of Fig. 4. 


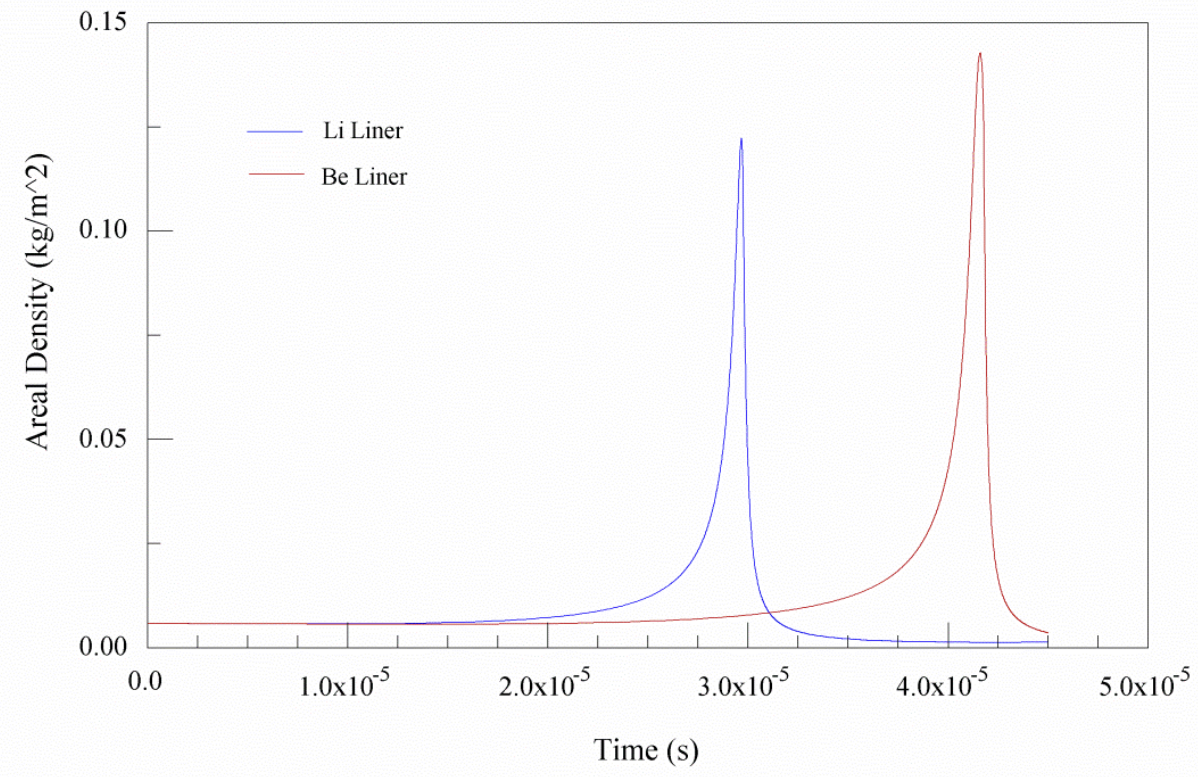

Fig. 23 Evolution of fuel areal density ( $\rho-R)$ with time

Figure 24 displays the cumulative fusion yield produced for each target; upon ignition, the D-T fuel rapidly generate energy in the form of $\alpha$-particles and neutrons, with a combined energy release of $17.6 \mathrm{MeV}\left(2.8 \times 10^{-12} \mathrm{~J}\right)$ per fusion event.

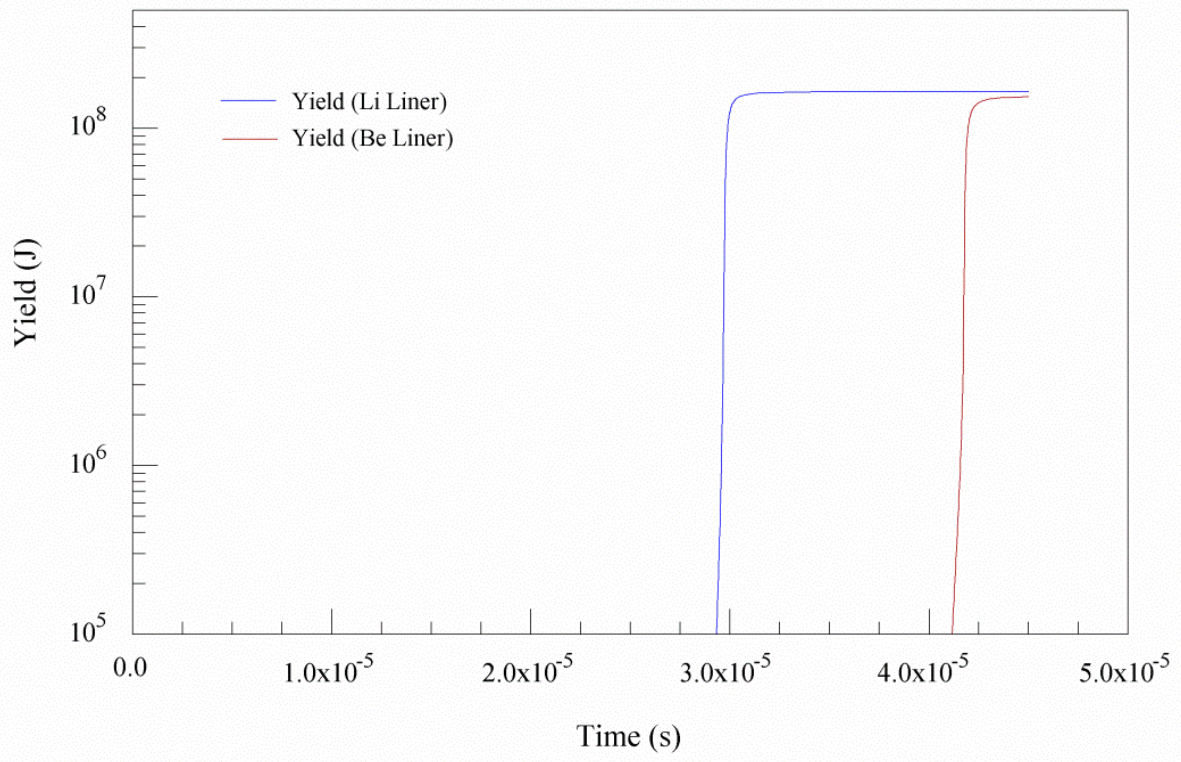

Fig. 24 Cumulative target yield as a function of time

For sufficiently strong internal magnetic fields, the $\alpha$-particle energy (3.5 MeV) is deposited in the fuel and contributes to a rapid increase in the fuel temperature; if not absorbed or reflected by the liner material, the 14.1 $\mathrm{MeV}$ neutron may escape the target and impact the surrounding magnet or support structure, depositing energy and causing embrittlement which must be taken into account in vehicle designs. The amount of neutrons produced during fusion, and the cumulative amount of neutrons produced in each compression cycle, are plotted in Figs. 25 and 26. 


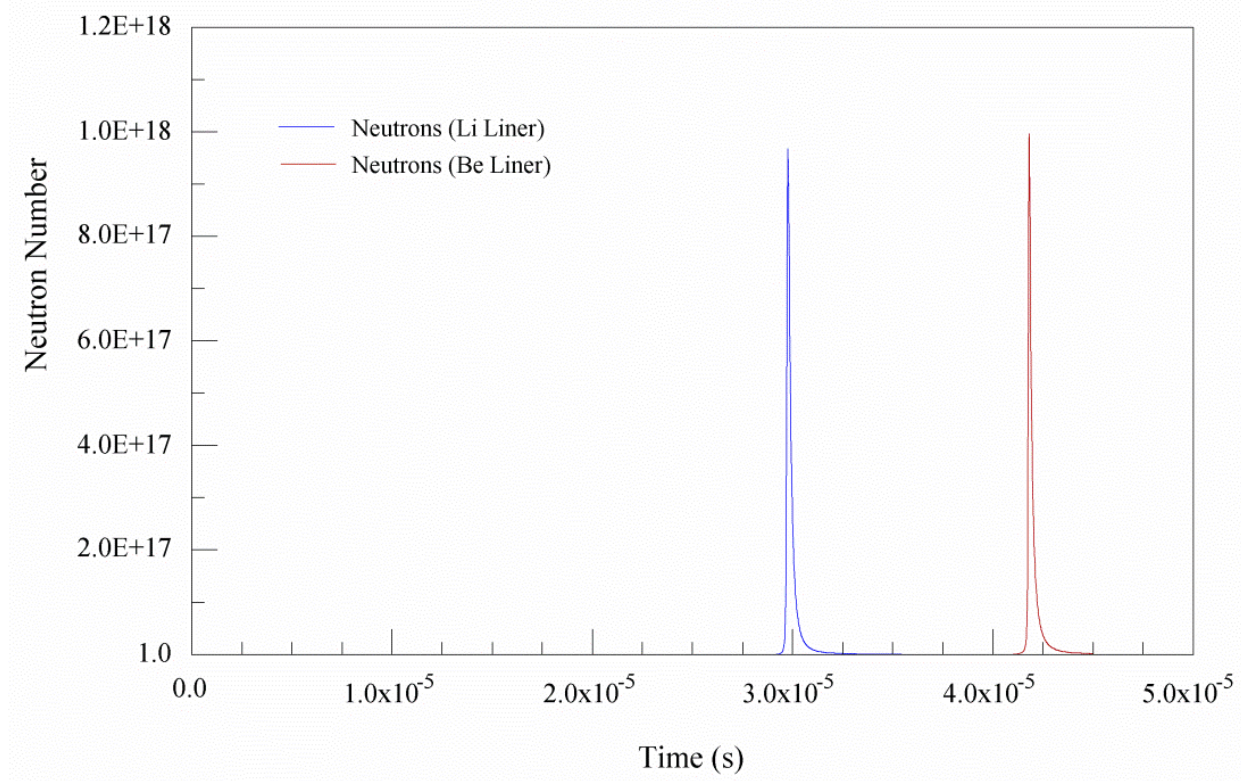

Fig. 25 Evolution of neutron production with time

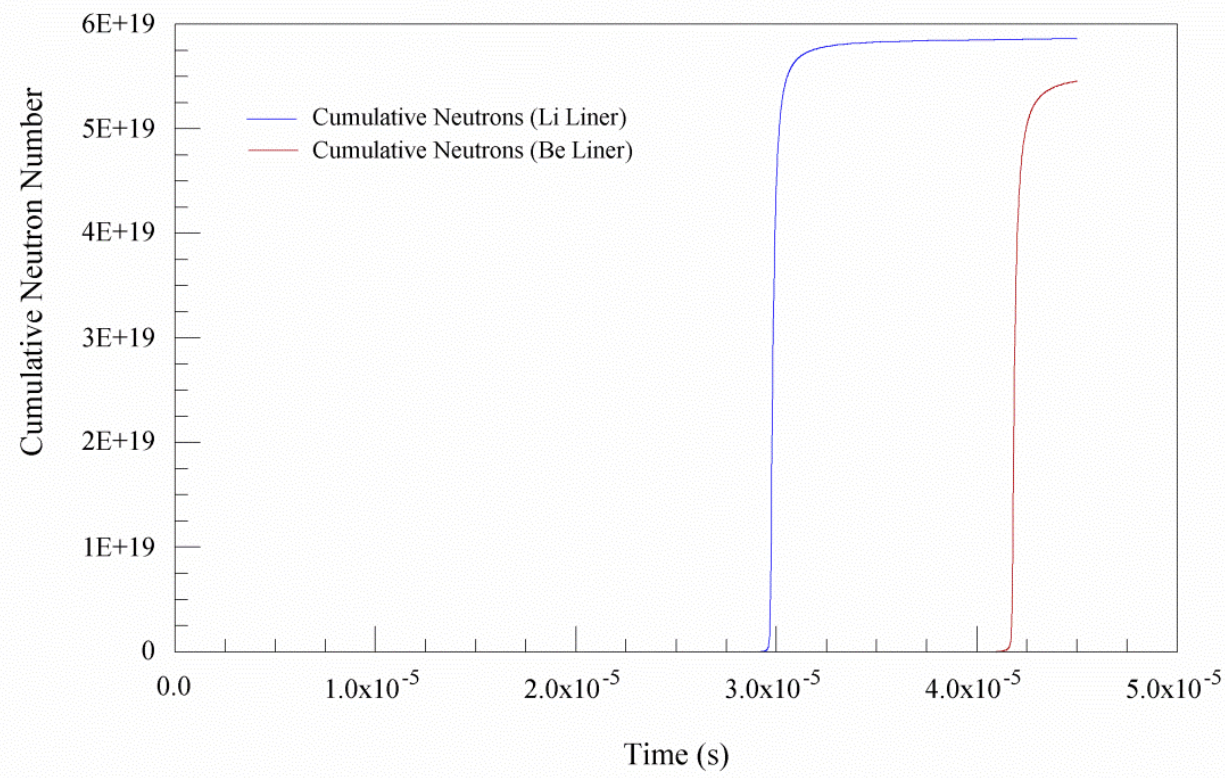

Fig. 26 Cumulative neutron production with time

In each case a copious amount of neutrons are produced, approximately $5.8 \times 10^{19}$ neutrons in the case of the $\mathrm{Li}$ lined target and $5.4 \times 10^{19}$ neutrons for the Be lined target. The energy carried by these neutrons is $1.3 \times 10^{8}$ and $1.2 \times 10^{8} \mathrm{~J}$, respectively, representing a significant deposition of energy into the surrounding structure during each pulse.

\section{Summary}

The key performance results of this section are summarized in Table 4 below, which displays the optimized target and magnetic field values assumed in these final analytic runs and the corresponding estimates of engine performance. Although the results are undoubtedly optimistic given the simplifying assumptions made in the model, they serve as an initial guide for more detailed numerical simulations to follow. 
Table 4. Engine performance estimates for optimized target and magnetic field values

\begin{tabular}{|c|c|c|c|c|c|c|c|}
\hline $\begin{array}{c}\text { Initial } \\
\text { Fuel } \\
\text { Density }\end{array}$ & $\begin{array}{c}\text { Initial } \\
\text { Liner } \\
\text { Radius }\end{array}$ & $\begin{array}{c}\text { Aspect } \\
\text { Ratio }\end{array}$ & $\begin{array}{c}\text { Injection } \\
\text { Velocity }\end{array}$ & $\begin{array}{c}\text { Fuel } \\
\text { Preheat } \\
\text { Temp }\end{array}$ & $\begin{array}{c}\text { External B- } \\
\text { Field }\end{array}$ & $\begin{array}{c}\text { Initial } \\
\text { Internal B- } \\
\text { Field }\end{array}$ & $\begin{array}{c}\text { Axial B- } \\
\text { Field } \\
\text { Gradient }\end{array}$ \\
\hline $0.07 \mathrm{~kg} / \mathrm{m}^{3}$ & $1.0 \mathrm{~cm}$ & 6 & $10 \mathrm{~km} / \mathrm{s}$ & $400 \mathrm{eV}$ & $30 \mathrm{~T}$ & $1.0 \mathrm{~T}$ & $100 \mathrm{~T} / \mathrm{m}$ \\
\hline \multicolumn{7}{|c|}{} \\
\hline \multicolumn{7}{|c|}{ Specific Impulse (s) } & \multicolumn{2}{|c|}{ Impulse (N-s) } & \multicolumn{2}{c|}{ Yield (J) } & \multicolumn{2}{c|}{ Gain (100\% efficiency) } \\
\hline Li Liner & Be Liner & Li Liner & Be Liner & Li Liner & Be Liner & Li Liner & Be Liner \\
\hline 32,200 & 17,145 & 780 & 1445 & $1.65 \times 10^{8}$ & $1.53 \times 10^{8}$ & 982 & 323 \\
\hline
\end{tabular}

\section{Preliminary Vehicle Design}

A preliminary vehicle design was evaluated as part of the initial study. As with most nuclear systems the vehicle design is dominated by the need to radiate waste heat, here mainly impinging on the struts and supports of the magnetic nozzle and main vehicle supports. Figure 27 provides an overview of the initial vehicle concept. The numbers shown in the figure reflect a fast trip to Mars carrying an Orion module and deep space habitat. The vehicle design leverages the prior analytical work found in [33,34]. The vehicle assumes a payload mass of $50 \mathrm{mT}$ and a (one-way) propellant mass of $50 \mathrm{mT}$, consistent with the mission analysis in Section VI. Table 5 in the following section provides the mass breakdown by subsystem, sized using the performance values from the previously mentioned references. Future work will include more detailed analysis of each individual subsystem.

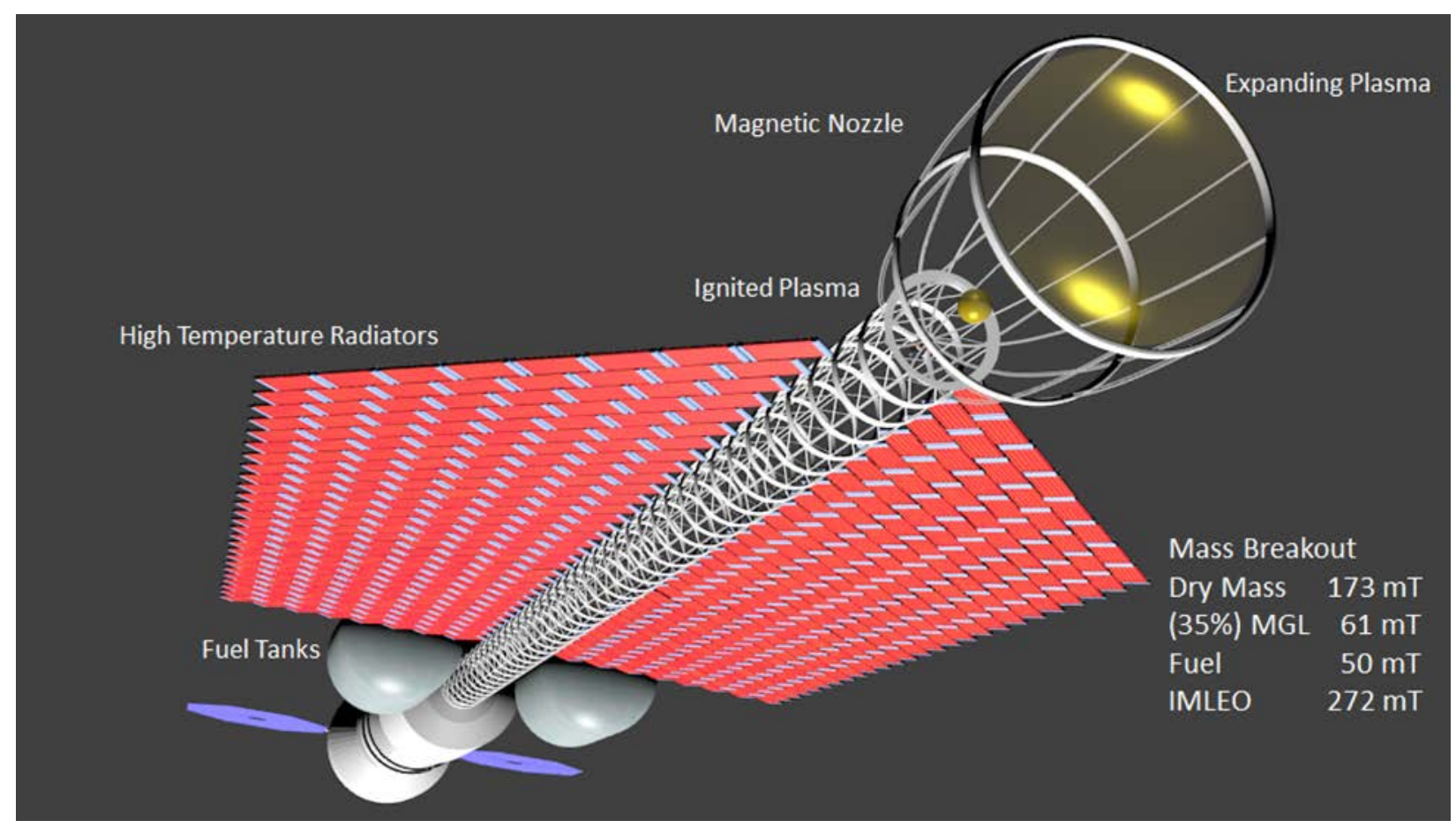

Fig. 27 Illustrative vehicle concept

\section{A. Vehicle Assumptions}

The propellant tanks are sized assuming a $10 \%$ mass relative to the amount of propellant stored. This is a fair approximation given the size of the tanks and the bulkiness of hydrogen. A reference point is the Shuttle external tanks, where the dry mass of the tanks is $7 \%$ of the fully loaded mass. 
Thermal subsystems can be complex to design because the size of the radiators are a strong function of the system efficiency. A rejection temperature of $1250 \mathrm{~K}$ is assumed in this analysis. The following steps are used to estimate the thermal load to be rejected. The jet power may be calculated as:

$$
\mathrm{P}_{\text {jet }}=\frac{1}{2} \dot{\mathrm{m}} \mathrm{V}_{\mathrm{e}}^{2}
$$

where $\dot{m}$ is the mass flow rate of propellant and Ve is the exhaust velocity. The thrust, F, is given by:

$$
\mathrm{F}=\dot{\mathrm{m}} \mathrm{V}_{\mathrm{e}}+\mathrm{A}_{\mathrm{e}}\left(\mathrm{p}_{\mathrm{e}}-\mathrm{p}_{\mathrm{a}}\right)
$$

where the second term is the exit area times the difference in exit pressure and atmospheric pressure; this term approaches zero for a well-designed nozzle. Substituting (57) into (56), and noting that the specific impulse may be written as $I_{s p}=\frac{F}{\dot{m g g}_{0}}$, yields the following expression for jet power:

$$
\mathrm{P}_{\text {jet }}=\frac{1}{2} \mathrm{Fg}_{0} \mathrm{I}_{\mathrm{sp}}
$$

For the lithium liner results shown in Table 4, operating the engine at a $10-\mathrm{Hz}$ repetition rate and assuming $100 \%$ efficiency provides a maximum jet power of approximately $1.2 \mathrm{GW}$. Thermal loads are primarily due to radiative heating of the superconducting magnet coils during target compression and fusion initiation, and radiation from the hot expanding plasma impinging on the struts that comprise the magnetic nozzle. The thermal load on the coils is expected to be relatively low, due to the short time frame of the pulsed fusion event, the rapid movement of the target through the coils into the nozzle, and the initial neutron reflector/moderator properties of the target liner. The plasma expanding into the magnetic nozzle region has a longer duration in which to impart thermal loads to the struts, hence the nozzle must be designed with as much open surface area as possible to allow the majority of photons and neutrons to escape, while still directing the charged plasma particles for thrust.

To calculate the thermal load, it is assumed that some fraction $\left(\varphi_{\text {therm }}\right)$ of the generated fusion power $\left(\mathrm{P}_{\text {gen }}\right)$ goes immediately into thermal losses to the coils and structure. Of the remaining power, some fraction ( $\varphi$ loss) is assumed to escape to space. The total power left in the directed plasma jet is then:

$$
\mathrm{P}_{\text {jet }}=\mathrm{P}_{\text {gen }}-\mathrm{P}_{\text {gen }} \varphi_{\text {therm }}-\mathrm{P}_{\text {gen }}\left(1-\varphi_{\text {therm }}\right) \varphi_{\text {loss }}
$$

For the initial calculations a $10 \%$ thermal loss is assumed ( $\left.\varphi_{\text {therm }}=0.1\right)$, with an additional $10 \%$ of the remaining energy lost to space $\left(\varphi_{\text {loss }}=0.1\right)$. Equation 59 then predicts $\mathrm{P}_{\text {jet }}=0.81 \cdot \mathrm{P}_{\text {gen }}$, or a loss of approximately $19 \%$. Assuming an initial power generation of $1.2 \mathrm{GW}$, the power loss is approximately $0.2 \mathrm{GW}$. These estimates are not unreasonable given the open structure of the magnetic nozzle, which provides a significant viewing area to the expanding plasma.

Using the standard equation for radiative heat transfer:

$$
\mathrm{q}=\varepsilon \sigma \mathrm{AT}^{4}
$$

where $\varepsilon$ is the infrared emissivity (optimistically set to unity), $\sigma$ is the Stefan-Boltzman constant, and $T$ is the rejection temperature $(1250 \mathrm{~K})$ yields a required viewing area, A, of approximately $1360 \mathrm{~m}^{2}$. The radiator mass can then be estimated assuming a double sided radiator with an areal density of $5 \mathrm{~kg} / \mathrm{m}^{2}$, as designed in [39], and a ratio of radiator mass to pump/cryo mass of unity, also from [39], to provide an estimated thermal mass of around 13.6 $\mathrm{mT}$.

The propulsion system is sized using an alpha of $17.6 \mathrm{~kW} / \mathrm{kg}$, which is the specific power used in the Magnetized Target Fusion (MTF) concept in [37]. This value is somewhat pessimistic, as the current system will be significantly less complex and presumably less massive than the referenced MTF system.

The structural mass fraction is assumed to be $10 \%$, calculated as a fraction of the total inert mass, which is a commonly used standard for in-space, medium thrust vehicles. Similarly the avionics mass is assumed to be $2 \%$.

Mass growth allowance is strongly debated in any new concept; here a mass growth allowance of $50 \%$ is assumed as an initial value, given the early stage of the propulsion and vehicle concept. 
Table 5. Preliminary Vehicle Design Parameters

\begin{tabular}{|c|c|}
\hline System & Mass (mT) \\
\hline Prop Tanks & 5.0 \\
\hline Thermal & 13.6 \\
\hline Propulsion & 75.4 \\
\hline Structural & 17.3 \\
\hline Avionics & 3.5 \\
\hline Mass Growth Allowance & 58.4 \\
\hline Inert Mass & 173.2 \\
\hline Payload & 50.0 \\
\hline Dry Mass & 223.2 \\
\hline Ullage & 1.5 \\
\hline Total Dry Mass & 224.7 \\
\hline Propellant & 48.5 \\
\hline IMLEO & 271.6 \\
\hline
\end{tabular}

\section{Mission Analysis}

In order to identify operating regimes with the greatest impact on space exploration missions, a parametric assessment of mission outcomes has been performed. To span both near term and far term mission goals, one-way missions to Mars and Saturn have been examined. In order to capture a range of applications from piloted to cargo, the missions spanned trip times from 20 days to one year, and with payloads from 20 metric tons (representing a crew vehicle for a fast transport) to 200 metric tons (representing a cargo mission in support of extended presence at the destination). While fusion offers the potential for much higher power and thrust levels than conventional electric propulsion, it is not quite in the range where simple impulsive $\Delta \mathrm{V}$ 's might be assumed. For demanding, fast planetary missions, the system must still thrust over a significant portion of the trajectory, which then introduces gravity and steering losses that must be accounted for based on system performance. In order to capture a possible range of operating scenarios, from nearly impulsive burns to extended operation similar to that of an electric propulsion vehicle, an analytic approximation method was used that captures this full range of operation.

The mission analysis is based on the equivalent length method developed by Zola [35], in which an equivalent length, derived from detailed trajectories over a range of acceleration levels, was found to be essentially invariant to the magnitude of the vehicle acceleration. Using this length, together with an assumption of travel in field free space, allows simplified rectilinear analysis of missions. This approach provides for rapid estimation of mission performance over a wide range of propulsion systems and mission times, and has been used previously to examine nuclear electric propulsion performance for Mars missions [36]. Equivalent length values for one way rendezvous missions to Mars and Saturn were used to scope vehicle performance for the current concept.

The overall propulsion performance of the system can be defined parametrically in terms of the specific power of the propulsion system, $\alpha(\mathrm{kW} / \mathrm{kg})$, the jet power, $\mathrm{P}_{\mathrm{j}}=\mathrm{F}^{2} / 2 \dot{\mathrm{m}}$, and specific impulse, $\mathrm{I}_{\mathrm{sp}}=\mathrm{F} / \dot{\mathrm{m}}_{0}$, where $\mathrm{F}$ is the thrust, $\dot{m}$ is the propellant mass flow rate, and $g_{0}$ is the acceleration of gravity at sea level.

Because calculations of the actual performance were concurrently being developed during the study, the system was initially modeled parametrically with values based on the PUFF fusion system [37], specifically using $\mathrm{P}_{\mathrm{j}}=1$ $\mathrm{GW}, \alpha=16 \mathrm{~kW} / \mathrm{kg}$, and varying $\mathrm{I}_{\mathrm{sp}}$ from 10,000 to 50,000 seconds. Using the equivalent length method described above, a calculation of initial mass and trip time could be performed for varying payloads, initial masses, and $\mathrm{I}_{\text {sp. }}$. In this way, the regimes in which the engine has the greatest impact can be identified and used to focus design efforts.

The results of these estimates are shown in Fig. 28 (Mars) and 29 (Saturn). Three dimensional contours of delivered payload mass are shown for 20, 50, 100, 150, and $200 \mathrm{mT}$ of payload, as functions of $\mathrm{I}_{\mathrm{sp}}$, initial mass $\left(\mathrm{M}_{0}\right)$, and trip time. The overall capability and limits of the system for both missions is evident from the limits of the contours. For Mars, the initial mass is fairly insensitive to trip time of Isp for trip times longer than 100 days, and approximately linear with payload mass. For shorter trip times, a minimum trip time of 40 days is possible for rapidly increasing propellant mass. Fast trips can be accomplished for initial masses of 200-500 mT, and at values of Isp below 20,000 s. 
The Saturn mission is more constrained, as would be expected for the greater distance. The minimum trip time is on the order of 200 days one way - a relatively short time compared to current propulsion options, but still representing significant exposure to space. A minimum Isp of 20,000 s is required to reach Saturn; at the lower payload mases, there is a lower sensitivity to higher Isp, as seen in the Mars case.

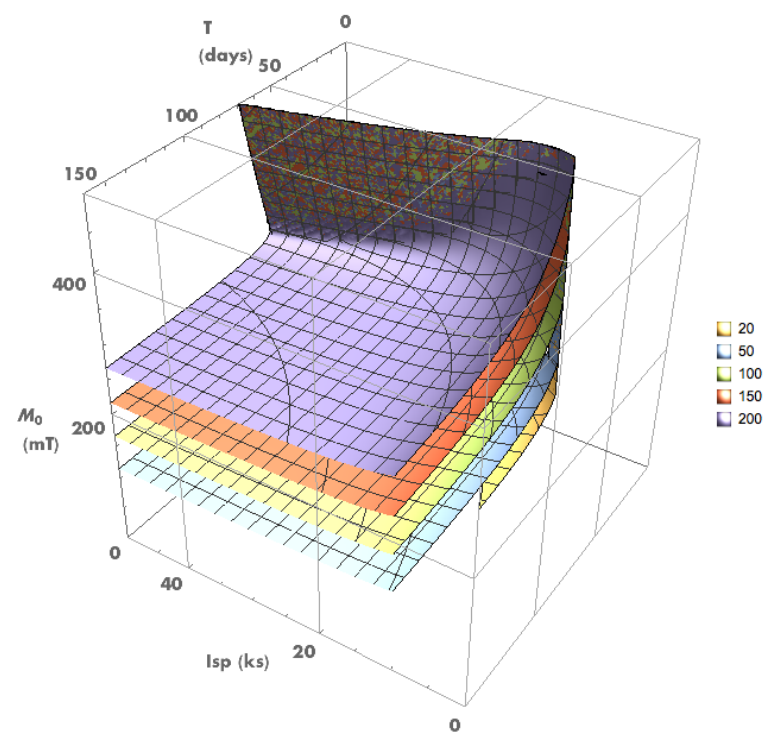

Fig. 28 Mission performance contours for one way trip to Mars.

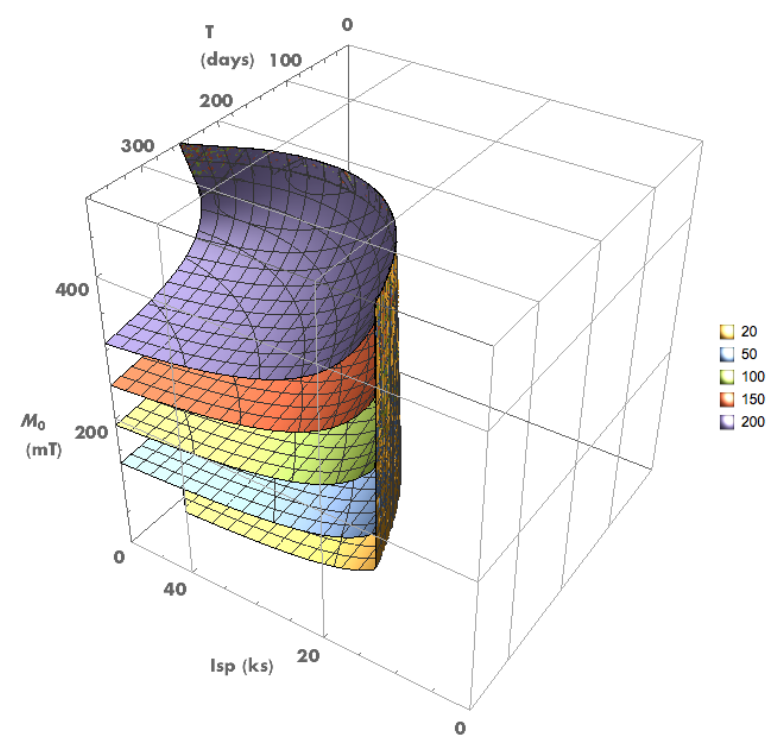

Fig. 29 Mission performance contours for one way trip to Saturn.

Summarizing the general benefits of the system, the preceding mission analysis indicates that the projected system performance can achieve impressively short trip times for both Mars and Saturn:

- Mars: 100-150 mT payload from LEO using 2 SLS block 2 launches (260 mT; [38]), < 60-90 days one-way trip time

- Saturn: 50-100 mT payload from LEO using 2 SLS block 2 launches, 200 days one-way trip time

\section{A. Detailed Mission Examples}

To provide more detailed performance estimates, the "optimized" performance values developed with the simplified semi-analytic model were used; as noted in Table 6, these correspond to the two thrust and specific impulse levels for the beryllium and lithium liners, but assumes comparable power levels. It is also assumed the engine operates at a $10-\mathrm{Hz}$ repetition rate to provide the average thrust values used in the table. These options were examined parametrically for a Mars outbound case over a range of trip times to give an estimate of their capability both for fast delivery for crewed missions and for high payload delivery. A crewed mission is assigned to primarily carry crew as the payload, with an estimated payload mass of $50 \mathrm{mT}$. The high payload mission assumes significant infrastructure will be delivered to the Martian surface.

Table 6. Calculated performance space for Gradient Field Fusion System

\begin{tabular}{c|c|c}
\hline & Li Liner & Be Liner \\
\hline Specific Impulse (s) & 32200 & 17145 \\
\hline Impulse (N-s) & 780 & 1445 \\
\hline Thrust (N) & 7800 & 14450 \\
\hline Yield (J) & $1.65 \times 10^{8}$ & $1.53 \times 10^{8}$ \\
\hline Gain (100\% efficiency) & 982 & 323 \\
\hline
\end{tabular}


To address this range of missions, payloads of 50, 100, and $200 \mathrm{mT}$ to Mars were considered, and propellant mass and total initial mass were calculated for the mission. These results are shown in Figs. 30 and 31 below. The fastest trip times are constrained by the all propulsive limit, in which the vehicle is constantly accelerating for the entire trip. Note that the overall propellant mass rapidly diminishes with trip time, to the point that vehicle mass becomes essentially linear with payload mass as the propellant mass asymptotically decreases to values less than either the payload or propulsion system. The Be liner case, with lower Isp, is capable of somewhat shorter trip times, although not dramatically shorter, at the cost of essentially twice the propellant mass.

Because of the high performance of the system and it's relative insensitivity to Mars mission times, a Saturn mission was also considered for the same system design parameters, as shown in Figs. 32 and 33. For one way trip times under a year, there is a greater sensitivity of propellant mass due to the higher energy requirements. There is also a significant benefit in using the Li liner system, with its higher Isp; propellant masses are substantially reduced, while accessible trip times are essentially the same. For a long term exploration program of the solar system, the Li liner system appears to offer the widest benefits, due to the high Isp and high power density of the fusion system.

The calculations to date have been for one way trips; however, especially in the case of the Mars mission, propellant mass plays a relatively small role in the overall system mass over a range of trip times, from $20 \mathrm{mT}$ ( $\mathrm{Li}$ liner) to $50 \mathrm{mT}$ (Be liner). A symmetric Mars round trip for a $50 \mathrm{mT}$ crew mission payload could be considered as an outbound payload of $100 \mathrm{mT}$, with $50 \mathrm{mT}$ used for the return trip. For an assumed vehicle mass of $320 \mathrm{mT}$, a $100 \mathrm{mT}$ payload using $50 \mathrm{mT}$ of propellant would require 45 days with the Li lined target system, and on the order of 75 days with the Be lined target system. For a lower vehicle mass of $270 \mathrm{mT}$, a $50 \mathrm{mT}$ payload can be delivered in 40 days (one way) with $50 \mathrm{mT}$ of propellant. Additional analysis with more detailed trajectory codes can be used to more accurately calculate potential round trip times, but assuming the validity of the semi-analytic model performance predictions, the engine appears capable of providing rapid transit for both crew and cargo to solar system destinations of current interest for human exploration.

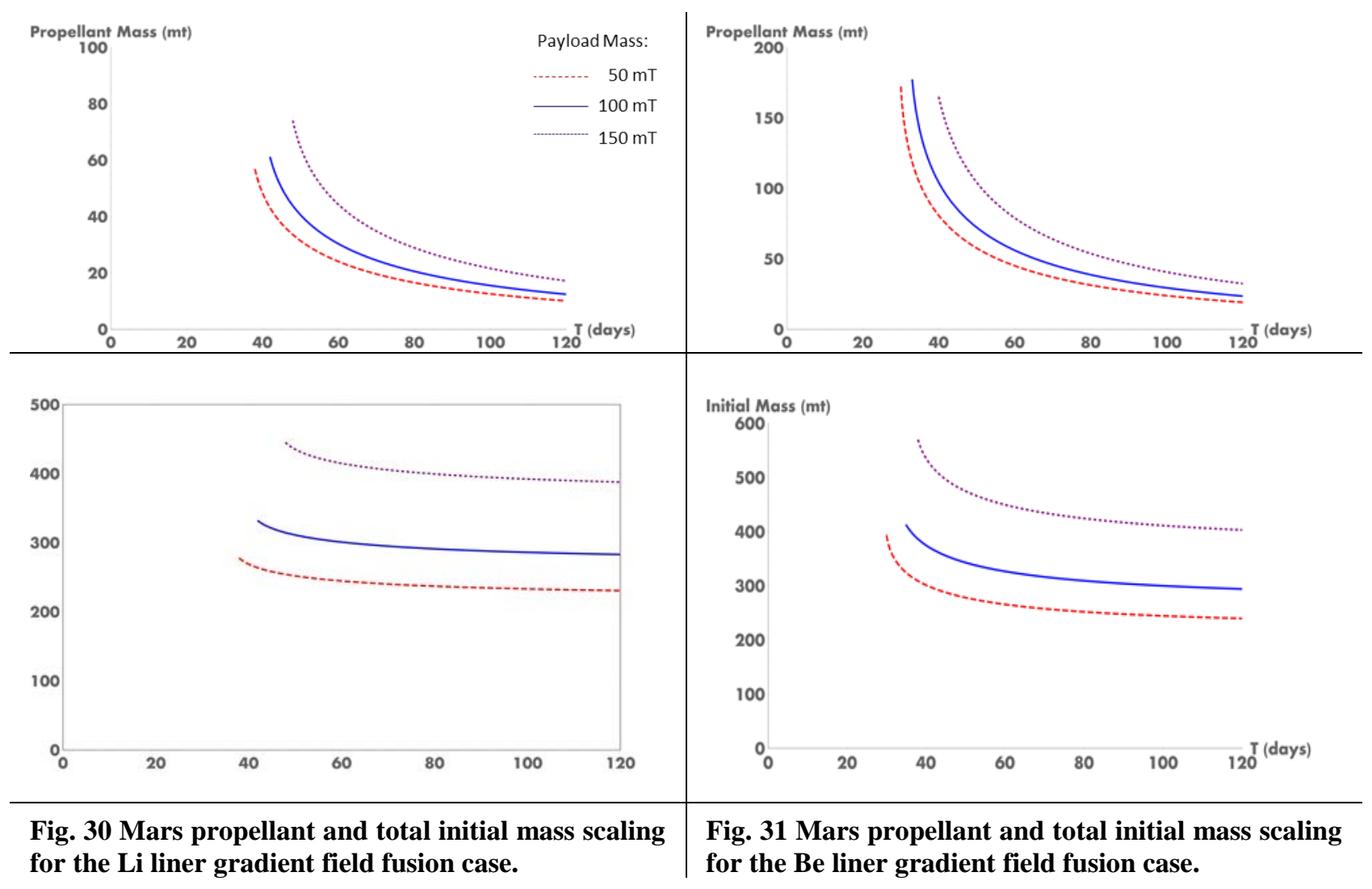




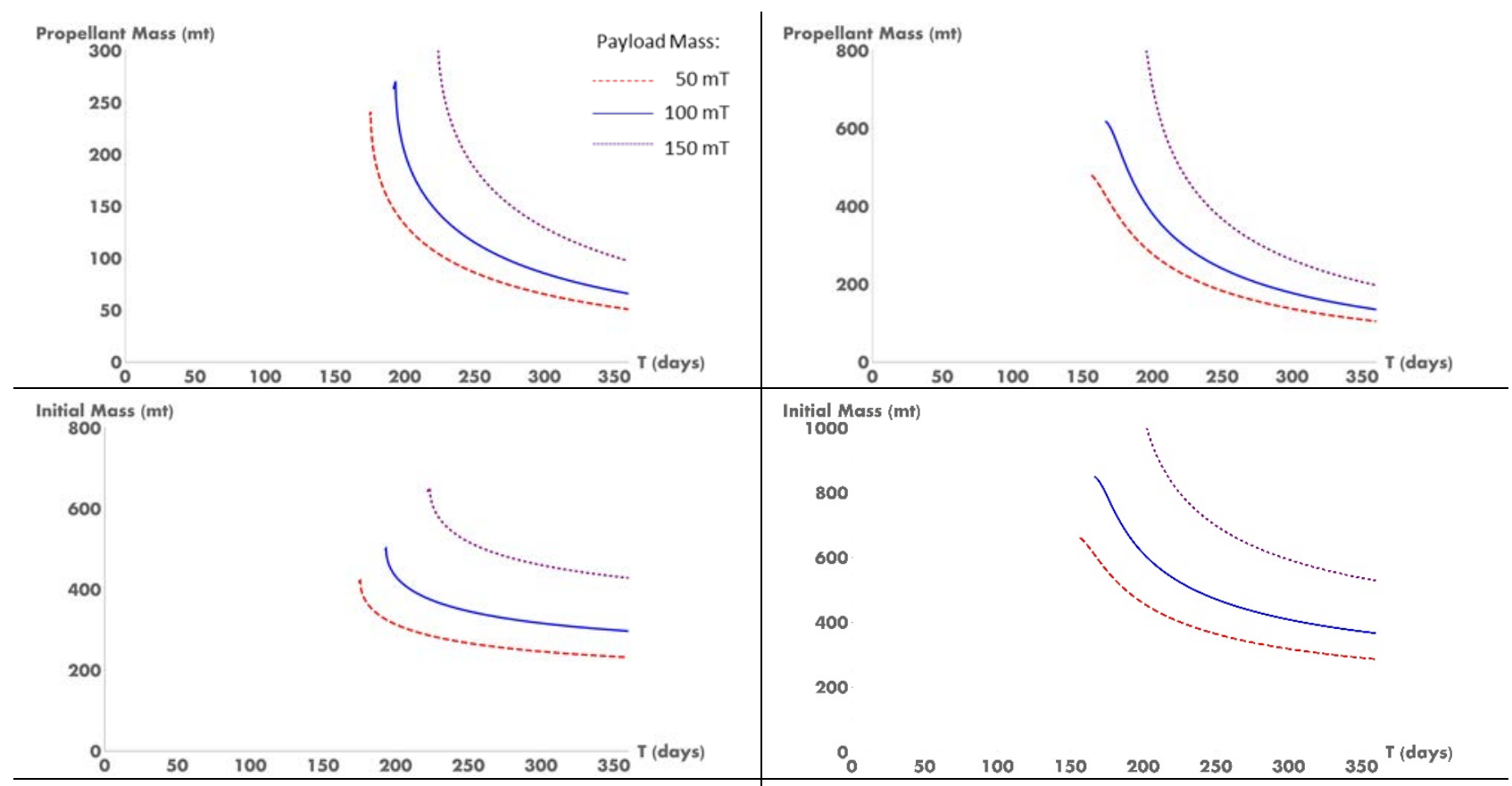

Fig. 32 Saturn propellant and total initial mass scaling for the $\mathrm{Li}$ liner gradient field fusion case.

Fig. 33 Saturn propellant and total initial mass scaling for the Be liner gradient field fusion case.

\section{Options for a Ground-Based Proof of Concept Demonstration}

Toward the end of the study, consideration was given toward a future ground-based experimental program to anchor the analytic and computational models for magnetic flux and target compression. Prior to significant expenditures on the accelerator and superconducting magnet systems outlined in this paper, suitable low cost hardware may be used to perform proof of concept tests to demonstrate the dynamics of radial compression arising from high velocity target injection into a suitably strong magnetic field. In place of the repetitively pulsed high power laser proposed for target ablation and acceleration, a ground based light gas gun can be used to generate high initial axial target velocities. Pulsed or steady state water-cooled coils can be used to generate a range of axial magnetic field strengths and gradients. Replacing the lithium or beryllium lined DT fusion target with more mundane but readily manufactured hollow or filled spheres and cylinders can provide data for comparison with target compression simulations. Bringing these available systems together into a ground based test facility for parametric testing and model validation would provide an economical first step toward demonstrating the feasibility of the concept, and if successful could lead to a more detailed experimental program. The following sections provide initial thoughts on a potential approach toward a ground based proof of concept demonstration.

\section{A. Two-Stage Light Gas Gun}

A two-stage light gas gun is a method of compressing and heating a working fluid (helium or hydrogen gas) to very high temperatures and pressures for the purpose of accelerating a projectile to very high velocities, greater than $7 \mathrm{~km} / \mathrm{sec}$. The general concept is to replace the combustible byproducts of a single stage propellant gun with a lower molecular weight gas, such as helium or hydrogen, resulting in a higher sound speed and ultimately higher velocity potential than the heavier gases from traditional systems. Figure 34 shows the major components and firing sequence of a typical tw0-stage light gas gun.

The University of Alabama Huntsville (UAH) operates the Aerophysics Research Center (ARC), located on the Redstone Arsenal in Huntsville, AL. The ARC houses three separate two-stage light gas gun systems, with associated test chambers and instrumentation, which are used to conduct experiments in the sub, super and hypersonic velocity regimes both for high speed flight and impact testing. The ARC also maintains several custom single-stage launchers systems that can be utilized or modified to meet specific user requirements. The intermediate two-stage light gas gun, with approximately $30-\mathrm{m}(100-\mathrm{ft})$ of flight range chambers and optional camera/data ports, appears suitable for a ground based proof of concept demonstration. A photograph of the intermediate light gas gun facility is provided in Fig. 35. Key parameters of the two-stage light gas guns available for this research are defined 
in Table 7. Previously tested projectiles on the intermediate light gas gun have achieved flight velocities on the order of 5-km/sec. For the purposes of studying flux compression, the projectiles could be spherical, cylindrical, conical and either solid, filled or hollow in construction. Various liner materials and shell thicknesses can be used to provide a parametric comparison against numerical simulation compression results.

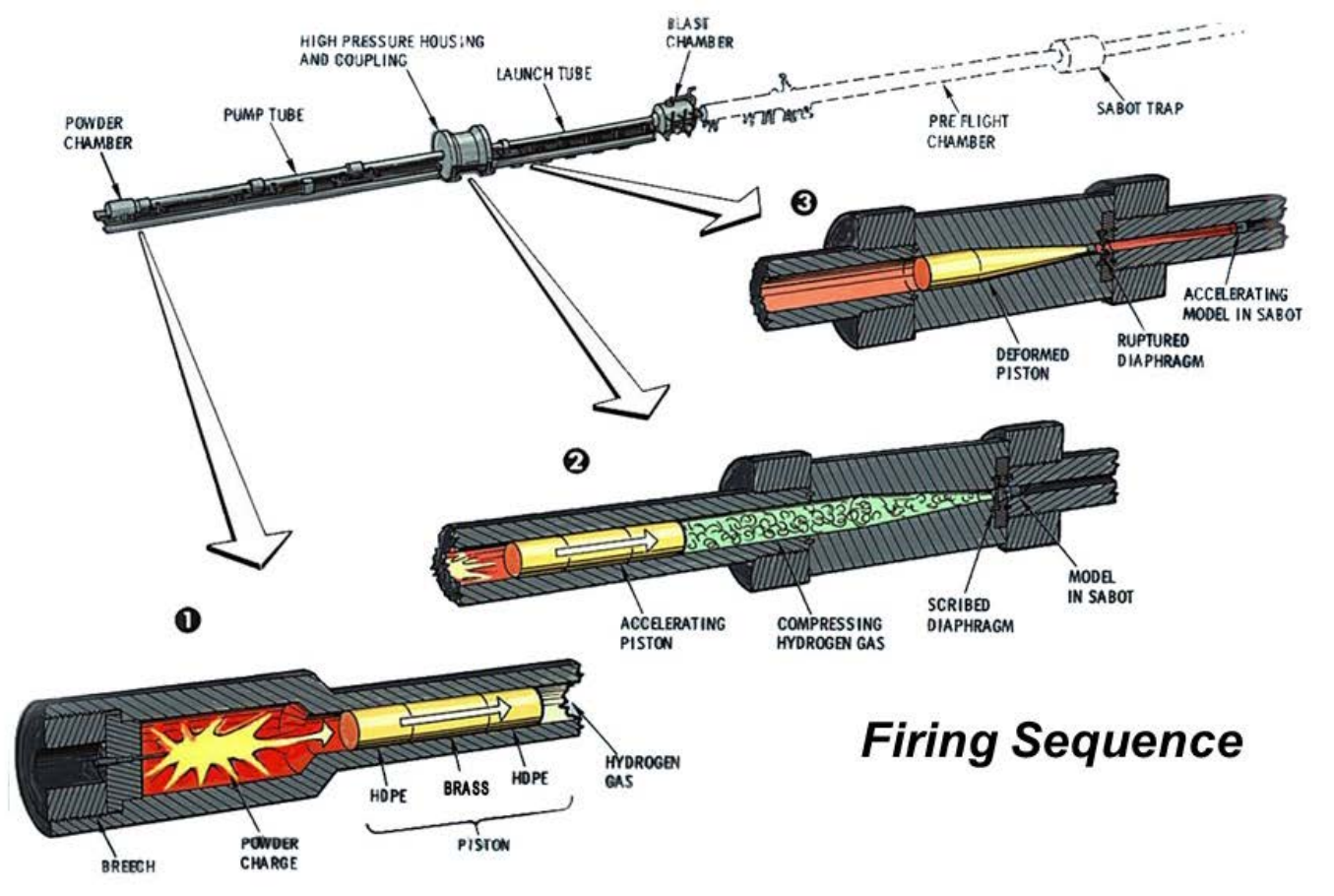

Fig. 34 Steps in Light Gas Gun firing sequence

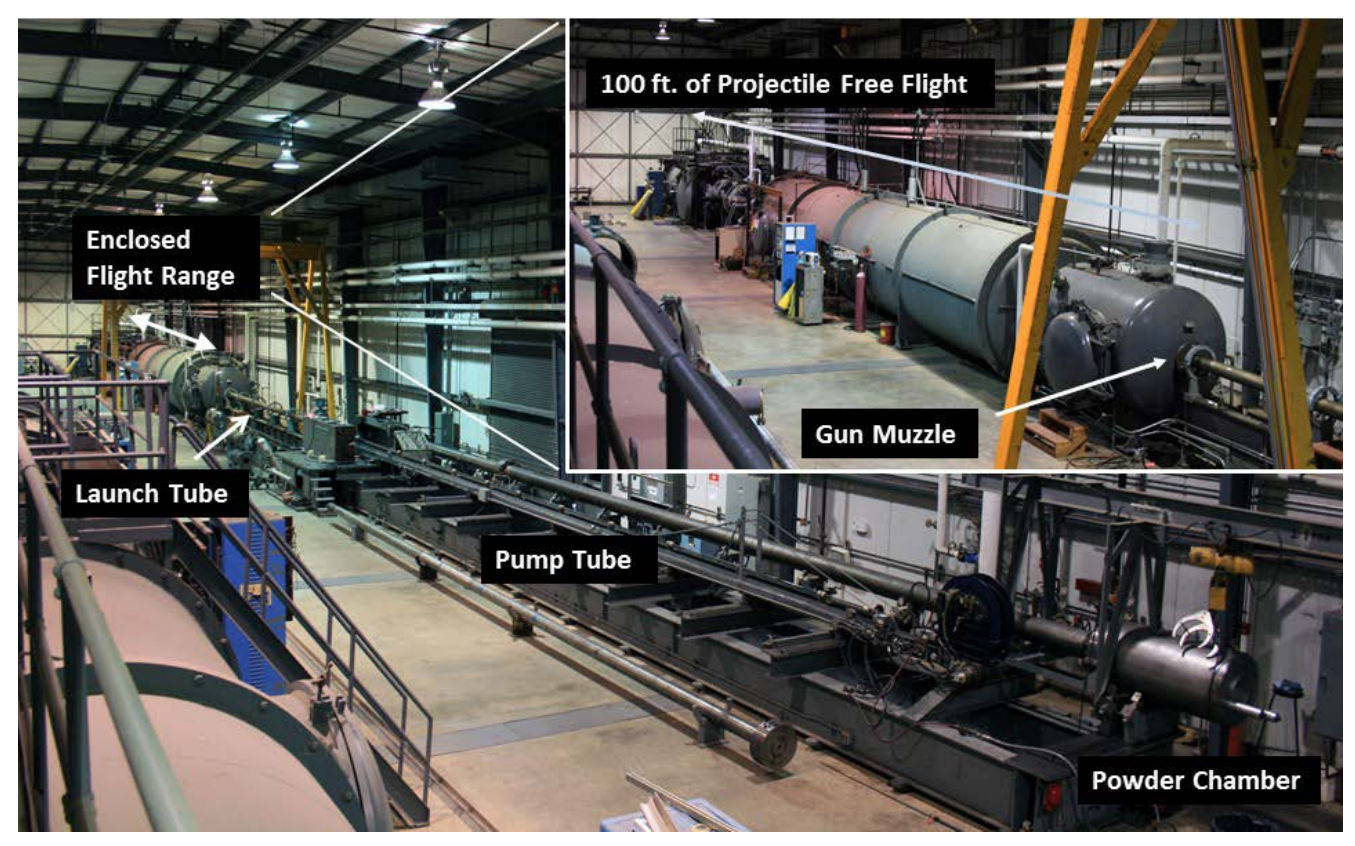

Fig. 35 Intermediate Sized Two-Stage Light Gas Gun Parameters 
Table 7. UAH ARC Two-stage Light Gas Gun parameters, including single-stage example

\begin{tabular}{|l|c|c|c|c|c|c|c|}
\hline $\begin{array}{c}\text { UAH ARC } \\
\text { Launcher } \\
\text { Systems }\end{array}$ & $\begin{array}{c}\text { Pump Tube } \\
\text { Length }\end{array}$ & $\begin{array}{c}\text { Pump Tube } \\
\text { Inside } \\
\text { Diameter }\end{array}$ & $\begin{array}{c}\text { Launch } \\
\text { Tube } \\
\text { Length }\end{array}$ & $\begin{array}{c}\text { Available Launch } \\
\text { Tube Inside } \\
\text { Diameters }\end{array}$ & $\begin{array}{c}\text { Primary Impact } \\
\text { Chamber }\end{array}$ & $\begin{array}{c}\text { Projectile } \\
\text { Launch Mass } \\
\text { Range }\end{array}$ & $\begin{array}{c}\text { Projectile } \\
\text { Velocity } \\
\text { Range }\end{array}$ \\
\cline { 2 - 8 } & $(\mathrm{m})$ & $(\mathrm{mm})$ & $(\mathrm{m})$ & $(\mathrm{mm})$ & $\begin{array}{c}\text { Diam x Length } \\
(\mathrm{m})\end{array}$ & $(\mathrm{gm})$ & $(\mathrm{km} / \mathrm{sec})$ \\
\hline Large & 38.13 & 254 & 22.88 & $\begin{array}{c}56,57,68,70,75, \\
78,86,100,152\end{array}$ & $3 \times 12.5$ & $150-12,000$ & $1-7.5$ \\
\hline Intermediate & 18.3 & 133 & 15.25 & $18,29,35$ & $2.4 \times 6.7$ & $40-250$ & $1-7.5$ \\
\hline Small & 13.42 & 108 & 7.47 & 19,29 & $1.8 \times 4.3$ & $10-130$ & $1-7.5$ \\
\hline Single Stage & NA & NA & 9.9 & 32 & $2.4 \times 6.7$ & $5-100$ & $0.1-1.1$ \\
\hline
\end{tabular}

\section{B. Potential Ground Test Setup}

An illustration of a potential test setup for measuring magnetic flux compression of a hypervelocity projectile is shown in Fig. 36. In this example, a 2-foot (0.6-m) diameter test chamber is fitted with single or multiple water cooled hollow copper coils to generate a static magnetic field using a high current DC source; alternatively, a pulsed current coil with capacitive power bank could be used and timed to provide a maximum quasi-steady (millisecond time scale) magnetic field just prior to and during the rapid traverse of the injected target. An instrument feedthrough is provided for the installation of B-dot probes to measure the dynamic change in the magnetic flux from the projectile. Additional instrumentation could include high speed cameras, suitable for time of flight tracking and centerline radial compression videography; wireless pressure sensors to provide compression measurements within the target; external measurement options such as flash $\mathrm{x}$-ray imaging during compression, and multiple data acquisition and control systems which can be readily modified for use. Although just a cursory look at instrumentation options was performed toward the close of the current study, it appears that several readily available sensors are available and could be included as part of a future experimental proof of concept effort.

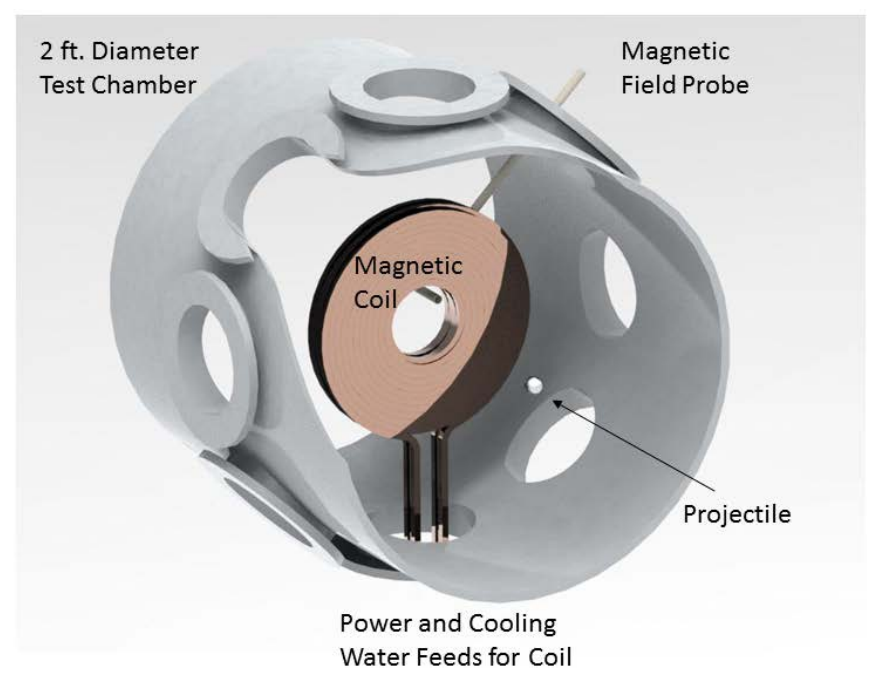

Fig. 36 Magnetic flux and target compression test setup

The ability to perform target compression tests with available ground facilities and equipment provides a relatively low cost approach for proof of concept testing of the target-field interaction underpinning the proposed concept. Using a light gas gun to inject a high speed target into a strong magnetic field produced by water cooled coils can economically map multiple parametric conditions against which the numerical models can be validated, with the results extrapolated to more accurately model this promising in-space propulsion system. Although not as well developed, a simultaneous program to evaluate pulsed material ablation from a target surface could also be 
carried out with commercially available $\mathrm{kW}$-class lasers and available, highly accurate impulse thrust stands, to evaluate the momentum imparted to a known target mass as a function of laser intensity and ablative material. The combination of ablative acceleration experiments and target compression experiments would help demonstrate the initial feasibility of the concept. As the terrestrial fusion community continues the development of MIF targets and the compression conditions required for gainful energy production, their results can be readily incorporated into the proposed system to refine target designs, magnetic field strengths and geometries, and acceleration requirements for a realistic, fusion based propulsion system.

\section{Concluding Remarks}

Human exploration of the solar system beyond our immediate neighborhood requires significantly faster transit times than offered by current or near term in-space propulsion systems. Crewed missions to Mars, Saturn, or other deep space destinations must balance the benefits of exploration against concerns for crew health and survivability; at present there are no implementable countermeasures against the high energy radiation associated with solar flares and cosmic radiation, or the deleterious effects of long duration weightlessness on the human body. Reducing astronaut trip times will help mitigate these survivability issues, while simultaneously reducing crew logistics mass and overall mission cost. Deep space robotic missions such as the notional Thousand Astronomical Unit mission will also benefit from more rapid trip times by delivering instruments and providing data within the career lifetimes of the scientists at home.

This study has evaluated the dynamics associated with a novel magnetized target fusion system proposed for rapid solar system exploration. Replacing the standard $\theta$-pinch geometry of a static target within a rapidly rising axial magnetic field to generate radial compressive forces, the concept under investigation injects a target at high axial velocity into a static axial magnetic field, creating similar radial compression forces in a geometry more suitable for in-space propulsion. During the study, a semi-analytic model was developed to simulate the key physics of target injection, radial liner compression, and conditions for gainful fusion energy release. A number of simulations were performed to define optimum target and field conditions, which were used to inform mission analysis and vehicle design studies. Concurrently, as described in a separate conference paper, substantial progress was made in the development of a significantly more accurate 3D physics based simulation, which will be used to further refine the target and field conditions required for sensible fusion gain. Preliminary results of the advanced simulation have demonstrated that liner currents are indeed induced due to rapid target injection into a strong magnetic field, underpinning the fundamental physics of the concept. Using the results of the analytic model, mission analysis has been performed for both Mars and Saturn missions, showing significantly reduced trip times for crewed missions and the capability for substantial cargo delivery supporting sustained exploration. Preliminary vehicle designs have been developed based on estimated parameters for the proposed system, forming a baseline against which more detailed designs will be developed as the modeling results are further refined. The rapid human exploration of destinations within our solar system requires significantly more energetic propulsion systems than current chemical engines or nuclear thermal propulsion can provide. The pulsed fusion propulsion system described in this paper may offer a practical, viable method to achieve the accelerations required for fast interplanetary transits. If successful, the concept will enable a new class of crewed exploration missions less constrained by the frailties of the human physique, opening the solar system to our ever expanding need for human exploration.

\section{Acknowledgements}

The authors gratefully acknowledge the financial support of the NASA Innovative Advanced Concepts Program in conducting this Phase I study. Our thanks to Dr. Ross Cortez, nou Systems, Inc., for several helpful discussions during the course of this activity.

\section{References}

[1] Murray, K.A.: “The Gevaltig: An Inertial Fusion Powered Manned Spacecraft Design for Outer Solar System Missions,” NASA-CR-185163, 1989.

[2] Orth, C. D., "VISTA - A Vehicle for Interplanetary Space Transport Application Powered by Inertial Confinement Fusion,” Lawrence Livermore National Laboratory, Final Systems Report UCRL-TR-110500, May 2003.

[3] Bond, A. and Martin, A., "Project Daedalus,” Journal of the British Interplanetary Society Supplement, Vol. 31, 1978, pp. S5-S7.

[4] Project Icarus website: http://icarusinterstellar.org/ 
[5] Williams, C. H., Dudzinski, L. A., Borowski, S. K., and Juhasz, A. J., “Realizing '2001: A Space Odyssey’: Piloted Spherical Torus Nuclear Fusion Propulsion,” NASA/TM-2005-213559, 2005.

[6] Slough, J. A., "Propagating Magnetic Wave Plasma Accelerator (PMWAC) for Deep Space Exploration,” NASA Institute for Advanced Concepts Phase I Final Report, 1999.

[7] Slough, J., et al., "Nuclear Propulsion through Direct Conversion of Fusion Energy: The Fusion Driven Rocket,” NASA Innovative and Advanced Concepts Phase I Final report, September 2012.

[8] Adams, R., Cassibry, J., Bradley, D., Fabisinski, L., Statham, G., “Pulsed Fission-Fusion (PuFF) - Phase I Report”, NASA Innovative and Advanced Concepts Final Report, 2013.

[9] Gibbs, W.W., “The Fusion Underground,” Scientific American, Nov 2016, pp. 38-45.

[10] Waldrop, M. M., “Plasma Physics: The Fusion Upstarts,” Nature, Vol. 511, July 2014, pp 398-400.

[11] Slutz, S. A., "Scaling Magnetized Liner Inertial Fusion on Z and Future Pulsed-Power Accelerators," Physics of Plasmas, Vol. 23, No. 2, Feb 2016, 022702.

[12] National Research Council, Committee on the Prospects for Inertial Confinement Fusion Energy, An Assessment of the Prospects for Inertial Fusion Energy, National Academies Press, 2013.

[13] Liberman, M. A., De Groot, J. S., Toor, A., and Spielman, R. B., Physics Of High Density Z Pinch Plasmas, Springer, 1999.

[14] Sefkow, A. B., Slutz, S. A., Koning, J. M., Marinak, M. M., Peterson, K. J., Sinars, D. B., Vesey, R. A., "Design of magnetized liner inertial fusion experiments using the Z facility,” Physics of Plasmas, Vol. 21, 2014, 072711.

[15] Miyamoto, K., Plasma Physics for Nuclear Fusion, MIT Press, Cambridge, MA, 1987.

[16] Cassibry et al., “Case and Development Path for Fusion Propulsion,” J. Spacecraft and Rockets, Vol. 52, No. 2, Mar-Apr 2015, pp. 595-612.

[17] Wurden, G. A. et al., “Magneto-Inertial Fusion,” J. Fusion Energy, Vol. 35, No. 16, 2016, pp 69-77.

[18] Gomez, M. R., et al., "Experimental Demonstration of Fusion-Relevant Conditions in Magnetized Liner Inertial Fusion,” Phys Rev Letters, Vol. 113. No. 15, 2004, pp. 155003-1-4.

[19] Awe, T.J., et al., "Experimental Demonstration of the Stabilizing Effect of Dielectric Coatings on Magnetically Accelerated Imploding Metallic Liners,” Phys. Rev. Lett., Vol. 116, No. 6, 2016, p. 065001-065006.

[20] Basko, M., Kempb, A., Meyer-ter-Vehn,J., "Ignition conditions for magnetized target fusion in cylindrical geometry," Nuclear Fusion, Vol. 40, No. 1, 2000, pp. 59-63.

[21] Freeland, R. M., “Project Icarus: Fission-Fusion Hybrid Fuel for Interstellar Propulsion,” J. British Interplanetary Society, Vol. 66, 2013, pp.290-296.

[22] J. Schonig, A. Ketsdever, and D. Kirtley, “Orbital Analysis of Macron Propulsion,” AIAA Paper 2010-6517, July 2010.

[23] Esmond, M.J.: Mostaghim, S.S.; Gebhart, T.E.; and Winfrey, A.L., "Investigation of Electrothermal Plasma Pellet Launcher Optimization for Fusion Fueling,” 2014 IEEE 41st International Conference on Plasma Sciences (ICOPS)/2014 IEEE International Conference on High-Power Particle Beams (BEAMS), May 25-29, 2014, DOI: 10.1109/PLASMA.2014.7012717,

[24] Felber, F. S., “Model of Ablative Acceleration at Moderate Laser Intensities,” Phys. Fluids, Vol. 23, No. 7, 1980, pp. 13801382.

[25] Jarboe, T. R., “Confinement and Acceleration of Pellet Material by One-Sided Laser Irradiation,” Phys. Fluids B, Vol. 5, No. 4, 1993, pp. 1332-1335.

[26] Jarboe, T. R., Kunkel, W. B., and Lietzke, A. F., "Study of Plasma Density Distribution Produced by Irradiating a 50- $\mu$ Deuterium Pellet on One Side With a Ruby Laser,” Phys. Fluids, Vol. 19, No. 10, 1976, pp. 1501-1506.

[27] National High Magnetic Field Laboratory, natinalmaglab.org. The world-record holding superconducting magnet has a cold bore size of 3.4-cm, weighs approximately 2.3 tons, and has an axial magnetic field strength of 32-T.

[28] Cassibry, Jason T, and S. T Wu, “Sub-Alfvenic Inlet Boundary Conditions for Axisymmetric MHD Nozzles,” Journal of Physics D: Applied Physics, Vol. 40, No. 17, 2007, pp. 5130-5139.

[29] Williams, C. H., "Application of Recommended Design Practices for Conceptual Nuclear Fusion Space Propulsion Systems,” AIAA Paper 2004-3534, July 2004.

[30] McBride, R. D. and Slutz, S. A., “A Semi-Analytic Model of Magnetized Liner Inertial Fusion,” Physics of Plasmas, Vol. 22, 2015, pp. 052708-1 - 052708-23.

[31] Slutz, S. A., Hermann, M. C., Vesey, R. A., Sefkow, A. B., Sinars, D. B., Rovang, D. C., Peterson, K. J., and Cuneo, M. E., "Pulsed power-Driven Cylindrical Liner Implosions of Laser Preheated Fuel Magnetized with an Axial Field,”. Physics of Plasmas, Vol. 17, 2010, pp. 056303-1 - 056303-15.

[32] Cassibry, J., Cortez, C., Cody, C., Thompson, S. and Jackson, L., "Three Dimensional Modeling of Pulsed Fusion for Propulsion and Terrestrial Power Using Smooth Particle Fluid with Maxwell Equation Solver (SPFMaX)", AIAA paper 2017-4677, July 2017.

[33] Adams, R. B., R. A. Alexander, J. M. Chapman, S. S. Fincher, R. C. Hopkins, A. D. Philips, T. T. Polsgrove, “Conceptual Design of In-Space Vehicles for Human Exploration of the Outer Planets,” NASA-TP-2004-213089, 2003. 
[34] Miernik, J., G. Statham, L. Fabisinski, C.D. Maples, R. Adams, T. Polsgrove, S. Fincher, “Z-Pinch Fusion-based Nuclear Propulsion,” Acta Astronautica, Vol 82, No. 2, 2013, pp. 173-182.

[35] Zola, C., "A Method of Approximating Propellant Requirements of Low- Thrust Trajectories," NASA TN D-3400, 1966.

[36] Gilland, J. H., "Mission and System Optimization of Nuclear Electric Propulsion Vehicles for Lunar and Mars Missions," NASA CR-189058, 1991.

[37] Robert B. Adams, Jason T. Cassibry, and Kevin Schillo. "Developing the Pulsed Fission-Fusion (PuFF) Engine", AIAA Paper 2014-3520, July 2014.

[38] National Aeronautics and Space Administration, Space Launch System, NASA Fact Sheet FS-2017-09-92-MSFC, 2017. 